\title{
INVARIANCE RESULTS FOR DELAY AND VOLTERRA EQUATIONS IN FRACTIONAL ORDER SOBOLEV SPACES
}

\author{
F. KAPPEL AND K. KUNISCH
}

\begin{abstract}
Invariance of the trajectories of infinite delay- and Volterra-type equations in fractional order Sobolev spaces are derived under minimal assumptions on the problem data. Properties of fractional order Sobolev spaces defined over intervals are summarized.
\end{abstract}

\section{Introduction.}

1.1. Problem statement and motivation. The problem under investigation is

$$
\begin{aligned}
x(t)= & \sum_{i=1}^{k} B_{i}(t) x\left(t-r_{i}\right)+\int_{-\infty}^{0} B(t, \sigma) x(t+\sigma) d \sigma \\
& +\sum_{i=0}^{k} \int_{s}^{t} A_{i}(\tau) x\left(\tau-r_{i}\right) d \tau+\int_{s}^{t} \int_{-\infty}^{0} A(\tau, \sigma) x(\tau+\sigma) d \sigma d \tau \\
& +f(t)+\eta \quad \text { for } t \in[s, T] \text { a.e., } \\
x(t)= & \phi(t-s) \quad \text { for } t<s \text { a.e., }
\end{aligned}
$$

where $0=r_{0}<r_{1}<\cdots<r_{k}=r<\infty, \eta \in \mathbf{R}^{n}, \phi$ and $x$ are $\mathbf{R}^{n}$-valued functions on $(-\infty, 0]$ and $[s, T], s<T$, respectively, and $B_{i}(t), A_{i}(t)$ and $B(t, \sigma), A(t, \sigma)$ are $n \times n$-matrix valued functions defined on $[s, T]$ and $[s, T] \times(-\infty, 0]$, respectively.

We observe that (1.1) includes as special cases Volterra integro-differential and integral equations of the type

$$
\dot{x}(t)=\int_{0}^{t} A(t-\sigma) x(\sigma) d \sigma, \quad t \in[0, T], x(0)=\eta,
$$

or

$$
x(t)=\int_{0}^{t} B(t-\sigma) x(\sigma) d \sigma, \quad t \in[0, T] .
$$

In recent years several detailed investigations of infinite delay equations have been carried out. We cite $[6,10,14,15,16]$ as representative publications.

It is well known that with $B_{i} \equiv 0, B \equiv A \equiv 0$, and $A_{i}$ constant the regularity of solutions increases with $t$. If the infinite delay terms (governed by $A$ and $B$ ) or the neutral terms (governed by $B_{i}$ and $B$ ) are nontrivial, then this smoothing effect is in general not preserved. But invariance of simple function spaces like $C$ or $L^{2}$ can

Received by the editors December 20, 1985 and, in revised form, August 13, 1986.

1980 Mathematics Subject Classification (1985 Revision). Primary 45D05, 45J05; Secondary 46E35.

Research in part supported by the Fonds zur Förderung der wissenschaftlichen Forschung, Austria, under \#S3206. 
be shown under appropriate assumptions on the problem data. Here, by invariance we mean that if $\phi$ is in some function space $F_{g}\left(-\infty, 0 ; \mathbf{R}^{n}\right)$ and if some matching conditions are met at $t=s$, then the solution is in $F\left(s, T ; \mathbf{R}^{n}\right)$ for arbitrary $T>s$. The subscript $g$ denotes a weighting factor related to the decay properties of $A$ and $B$ as $\sigma \rightarrow-\infty$. This will be made more precise further below.

The purpose of this paper is to study invariance results in Sobolev spaces, to show continuous dependence of the solutions on the problem data, as well as to develop the necessary results for fractional order Sobolev spaces on bounded intervals. Invariance results in these spaces are important, for instance, for approximation methods for (1.1), since convergence and especially rate of convergence depend on the smoothness of the function to be approximated [12]. The smoother the function is, the better is the rate of convergence in general. Smoothness of solutions, however, necessitates strong requirements on the regularity of the problem data. This can be seen below when we derive invariance results in $H^{m}$-spaces, $m=1,2, \ldots$ Recently, numerical schemes have also been used successfully in parameter estimation problems (cf. $[\mathbf{2}, \mathbf{3}, \mathbf{9}]$ ). In this case one might be interested in the other extreme: least possible smoothness such that at least convergence of an approximation scheme is guaranteed and such that only weak assumptions on the problem data are required.

As a specific example we have in mind approximation of (1.1) by first order spline functions with a convergence proof in a semigroup-theoretical setting similar to that in [3]. This requires convergence of first order spline interpolates $\phi^{N}$ to $\phi$ in the $H^{1}$-norm, which can be obtained for $\phi \in H^{1+\alpha}, \alpha>0$ (the definition of the spaces $H^{1+\alpha}, \alpha \in(0,1)$, is given below). This motivates the study of invariance of (1.1) in $H^{1+\alpha}$ especially for $\alpha \in\left(0, \frac{1}{2}\right)$, because invariance can be obtained under the assumption $A_{i} \in H^{\alpha}$, which does not require $A_{i}$ to be continuous.

In the semigroup approach to (1.1) it is necessary to choose a state space corresponding to the fact that the initial function $\phi$ evolves in time according to (1.1). A possible choice is $\mathbf{R}^{n} \times L_{g}^{2}\left(-\infty, 0 ; \mathbf{R}^{n}\right)$. Then the associated state is given by $\left(y(t), x_{t}\right), t \geq s$, where

$$
\begin{gathered}
y(t)=x(t)-\sum_{i=1}^{k} B_{i}(t) x\left(t-r_{i}\right)-\int_{-\infty}^{0} B(t, \sigma) x(t+\sigma) d \sigma \\
x_{t}(\tau)=x(t+\tau), \quad-\infty<\tau \leq 0 .
\end{gathered}
$$

By the results of [4] this state (or the state where $y(t)$ is replaced by $y(t)-f(t)$ if $f \not \equiv 0)$ is a natural choice. It will turn out that $t \rightarrow\left(y(t)-f(t), x_{t}\right)$ is continuous whereas $t \rightarrow\left(x(t), x_{t}\right)$ in general is not. The pair $(y(t), x(t))$ is governed by the following system equivalent to (1.1):

$$
\begin{aligned}
& y(t)=\eta+f(t)+\sum_{i=0}^{k} \int_{s}^{t} A_{i}(\tau) x\left(\tau-r_{i}\right) d \tau+\int_{s}^{t} \int_{-\infty}^{0} A(\tau, \sigma) x(\tau+\sigma) d \sigma d \tau, \\
& x(t)=y(t)+\sum_{i=1}^{k} B_{i}(t) x\left(t-r_{i}\right)+\int_{-\infty}^{0} B(t, \sigma) x(t+\sigma) d \sigma
\end{aligned}
$$

a.e. for $t \in[s, T]$,

$$
x(t)=\phi(t-s) \text { a.e. for } t<s .
$$


When approximating the state $\left(y(t), x_{t}\right)$ the problem arises to obtain a supremum norm approximation of $x(t)$ itself. Under additional smoothness assumptions this has been done for finite delay equations in [8]. These assumptions can be relaxed by using invariance of $H^{1+\alpha}$ for $\alpha \in\left(\frac{1}{2}, 1\right)$.

In deriving our results it will be necessary to make frequent use of the properties of fractional order Sobolev spaces. In spite of a wide literature on this subject we could not find many results in a form which was adequate for our purposes. Furthermore, several authors use different but equivalent definitions for fractional order Sobolev spaces. The equivalence of these various approaches is not always obvious for the nonspecialist. We therefore summarize and prove several of the properties of fractional order Sobolev spaces making use of the fact that in our case the domain of the functions has dimension one.

1.2. Sobolev spaces on bounded intervals. As already indicated in $\S 1.1$ we shall also use fractional order Sobolev spaces which sometimes are called Slobodeckij spaces. In the literature one can find a number of different characterizations for these spaces and usually functions defined on $n$-dimensional domains are considered. Here we are interested in the considerably simpler case of functions defined on an interval. On the other hand we need some basic facts concerning spaces with weighted norms and spaces of functions with values in a Hilbert space which are not directly available in the literature. For the convenience of the reader we shall give proofs or indications thereof when for the nonspecialist it is difficult to extract the proof from the literature.

1.2.1. Integer order Sobolev spaces. For a Hilbert space $X$ with norm $|\cdot|$ and a closed bounded interval $I$ let $L^{p}(I ; X)$ as usual be the equivalence class of functions $f: I \rightarrow X$ such that

$$
|f|_{L^{p}(I ; X)}=\left(\int_{I}|f(\tau)|^{p} d \tau\right)^{1 / p}<\infty, \quad 1 \leq p<\infty
$$

and

$$
|f|_{L^{\infty}}(I ; X)=\sup _{I} \operatorname{ess}|f(\tau)|<\infty .
$$

For $m=1,2, \ldots$ let $H^{m}(I ; X)=\left\{f \in L^{2}(I ; X) \mid f^{(j-1)}\right.$ is absolutely continuous on $I$ and $\left.f^{(j)} \in L^{2}(I ; X), j=1, \ldots, m\right\}$ and endow $H^{m}(I ; X)$ with the usual Hilbert space topology.

1.2.2. The spaces $H^{s}(\mathbf{R} ; X), s \geq 0$. First let $m \in \mathbf{N}$. We define $[\mathbf{1 1}$, p. 30] $H^{m}(\mathbf{R} ; X)=\left\{u \in L^{2}(\mathbf{R} ; X) \mid u^{(j-1)}\right.$ locally absolutely continuous and $u^{(j)} \in$ $\left.L^{2}(\mathbf{R} ; X), j=1, \ldots, m\right\} . H^{m}(\mathbf{R} ; X)$ is a Hilbert space with the usual norm

$$
|u|_{H^{m}(\mathbf{R} ; X)}^{2}=\sum_{j=0}^{m}\left|u^{(j)}\right|_{L^{2}(\mathbf{R} ; X)}^{2} .
$$

Since the domain of definition for the functions considered here is $\mathbf{R}$, there is no need for introducing distributional derivatives (see for instance $[1$, p. $70 \mathrm{ff}]$ ). Let $\hat{u}=\mathcal{F} u$ denote the Fourier-transform of $u$,

$$
\hat{u}(y)=\frac{1}{\sqrt{2 \pi}} \int_{-\infty}^{\infty} e^{-i y t} u(t) d t, \quad y \in \mathbf{R} .
$$


Then $H^{m}(\mathbf{R} ; X)$ can also be characterized as

$$
H^{m}(\mathbf{R} ; X)=\left\{u \in L^{2}(\mathbf{R} ; X) \mid\left(1+|y|^{2}\right)^{m / 2} \hat{u} \in L^{2}(\mathbf{R} ; X)\right\},
$$

see $[12$, p. 5]. The norm

$$
|||u| \|_{H^{m}(\mathbf{R} ; X)}=\left|\left(1+|y|^{2}\right)^{m / 2} \hat{u}\right|_{L^{2}(\mathbf{R} ; X)}
$$

is equivalent to the norm defined in (1.7). The proof of this fact uses Plancherel's theorem which is also true for Hilbert space valued functions.

The characterization (1.8) of $H^{m}(\mathbf{R} ; X)$ suggests the definition of $H^{s}(\mathbf{R} ; X)$ for real numbers $s \geq 0$ (and even all $s \in \mathbf{R}$ ):

$$
H^{s}(\mathbf{R} ; X)=\left\{u \in L^{2}(\mathbf{R} ; X) \mid\left(1+|y|^{2}\right)^{s / 2} \hat{u} \in L^{2}(\mathbf{R} ; X)\right\} .
$$

The norm

$$
\||| u||_{H^{s}(\mathbf{R} ; X)}=\left|\left(1+|y|^{2}\right)^{s / 2} \hat{u}\right|_{L^{2}(\mathbf{R} ; X)}
$$

introduces a Hilbert space structure on $H^{s}(\mathbf{R} ; X)$.

An important result is that the spaces $H^{s}(\mathbf{R} ; X)$ can be characterized as interpolation spaces in the sense of $\left[\mathbf{1 1}\right.$, p. 31] between $H^{m}(\mathbf{R} ; X)$ and $L^{2}(\mathbf{R} ; X)$ :

$$
H^{s}(\mathbf{R} ; X)=\left[H^{m}(\mathbf{R} ; X), L^{2}(\mathbf{R} ; X)\right]_{\theta}, \quad s=(1-\theta) m .
$$

The interpolation norm of $H^{s}(\mathbf{R} ; X)$ is equivalent to the norm in (1.11).

We shall also use a characterization of the spaces $H^{s}(\mathbf{R} ; X)$ as intermediate spaces defined by semigroups (see $[11$, p. $48 ; \mathbf{5}]$ ). Let $T(t), t \geq 0$, be a uniformly bounded $C_{0}$-semigroup on $L^{2}(\mathbf{R} ; X)$ with infinitesimal generator $A$ and assume that $D\left(A^{m}\right)=H^{m}(\mathbf{R} ; X)$ for some $m \in \mathbf{N}$. Then (see [5, Theorem $3.4 .2 ; \mathbf{1 1}, \mathrm{p}$. 98])

$$
H^{s}(\mathbf{R} ; X)=\left\{\left.u \in L^{2}(\mathbf{R} ; X)\left|\int_{0}^{\infty} t^{-1-2 s}\right|(T(t)-I)^{m} u\right|_{L^{2}(\mathbf{R} ; X)} ^{2} d t<\infty\right\}
$$

for every $s \in(0, m)$. Moreover

$$
\|u\|_{H^{s}(\mathbf{R} ; X)}^{2}=|u|_{L^{2}(\mathbf{R} ; X)}^{2}+\int_{0}^{\infty} t^{-1-2 s}\left|(T(t)-I)^{m} u\right|_{L^{2}(\mathbf{R} ; X)}^{2} d t
$$

defines an equivalent norm on $H^{s}(\mathbf{R} ; X)$.

Let $s=k+\beta, k \in \mathbf{N}$ and $\beta \in(0,1)$. Then by the Theorem of Reduction [ 5 , Theorem 3.4.6] we have

$$
H^{s}(\mathbf{R} ; X)=\left\{u \in D\left(A^{k}\right) \mid A^{k} u \in H^{\beta}(\mathbf{R} ; X)\right\}
$$

with the equivalent norm

$$
\|u\|_{H^{s}(\mathbf{R} ; X)}^{2}=|u|_{D\left(A^{k}\right)}^{2}+\int_{0}^{\infty} t^{-1-2 \beta}\left|(T(t)-I) A^{k} u\right|_{L^{2}(\mathbf{R} ; X)}^{2} d t .
$$

Here $|\cdot|_{D\left(A^{k}\right)}$ denotes the graph norm of $A^{k}$. Note that only the behavior of $T(t) u$ for small $t$ is important in (1.16). In fact, if $\delta>0$ then

$$
\begin{aligned}
\int_{0}^{\infty} t^{-1-2 \beta} \mid(T(t) & -I)\left.A^{k} u\right|_{L^{2}(\mathbf{R} ; X)} ^{2} d t=\int_{0}^{\delta} t^{-1-2 \beta}\left|(T(t)-I) A^{k} u\right|_{L^{2}(\mathbf{R} ; X)}^{2} d t \\
& +\int_{\delta}^{\infty} t^{-1-2 \beta}\left|(T(t)-I) A^{k} u\right|_{L^{2}(\mathbf{R} ; X)}^{2} d t .
\end{aligned}
$$


Using $|T(t)| \leq M$ for some $M>0$ and all $t \geq 0$, the second integral can be estimated as

$$
\begin{aligned}
\int_{\delta}^{\infty} t^{-1-2 \beta}\left|(T(t)-I) A^{k} u\right|_{L^{2}(\mathbf{R} ; X)}^{2} d t & \leq(M+1)^{2}\left|A^{k} u\right|_{L^{2}(\mathbf{R} ; X)}^{2} \int_{\delta}^{\infty} t^{-1-2 \beta} d t \\
& \leq \frac{\delta^{-2 \beta}}{2 \beta}(M+1)^{2}|u|_{D\left(A^{k}\right)}^{2} .
\end{aligned}
$$

This implies the equivalence for any $\delta>0$ of the norm given in (1.16) and the following norm denoted by the same symbol:

$$
\|u\|_{H^{s}(\mathbf{R} ; X)}^{2}=|u|_{D\left(A^{k}\right)}^{2}+\int_{0}^{\delta} t^{-1-2 \beta}\left|(T(t)-I) A^{k} u\right|_{L^{2}(\mathbf{R} ; X)}^{2} d t .
$$

As a concrete semigroup we can take the shift semigroup on $L^{2}(\mathbf{R} ; X)$ defined by

$$
(T(t) u)(\tau)=u(t+\tau) \quad \text { for } t \geq 0, \tau \in \mathbf{R}, u \in L^{2}(\mathbf{R} ; X) .
$$

Obviously this defines a uniformly bounded $C_{0}$-semigroup. The infinitesimal generator of $T(t)$ is characterized by

$$
D(A)=H^{1}(\mathbf{R} ; X), \quad A u=\dot{u} \quad \text { for } u \in D(A) .
$$

In order to prove this let $\hat{T}(t)=\mathcal{F} T(t) \mathcal{F}^{-1}, t \geq 0$. Then $\hat{T}(t), t \geq 0$, is the $C_{0}$-semigroup on $L^{2}(\mathbf{R} ; X)$ given by

$$
(\hat{T}(t) \hat{u})(y)=e^{i y t} \hat{u}(y), \quad y \in \mathbf{R}, t \geq 0 .
$$

Direct computation shows that the infinitesimal generator $\hat{A}$ of $\hat{T}(t), t \geq 0$, is given by

$$
\begin{aligned}
D(\hat{A}) & =\left\{\hat{u} \in L^{2}(\mathbf{R} ; X) \mid y \hat{u} \in L^{2}(\mathbf{R} ; X)\right\}, \\
\hat{A} \hat{u} & =i y \hat{u}(y), \quad \hat{u} \in D(\hat{A}) .
\end{aligned}
$$

The characterization given above for $H^{1}(\mathbf{R} ; X)$ shows that $\hat{u} \in D(\hat{A})$ is equivalent to $u=\mathcal{F}^{-1} \hat{u} \in H^{1}(\mathbf{R} ; X)$. Moreover, $i y \hat{u}=\mathcal{F} \dot{u}$. This proves (1.18) if we observe $D(A)=\mathcal{F}^{-1} D(\hat{A})$ and $A=\mathcal{F}^{-1} \hat{A} \mathcal{F}$.

Using the shift semigroup, the characterization (1.15) and the norm (1.16) are equivalent to

$$
H^{s}(\mathbf{R} ; X)=\left\{u \in H^{k}(\mathbf{R} ; X) \mid u^{(k)} \in H^{\beta}(\mathbf{R} ; X)\right\}
$$

and

$$
\begin{aligned}
& \|u\|_{H^{s}(\mathbf{R} ; X)}^{2} \\
& \quad=|u|_{H^{k}(\mathbf{R} ; X)}^{2}+\int_{0}^{\infty} t^{-1-2 \beta} \int_{-\infty}^{\infty}\left|u^{(k)}(\tau)-u^{(k)}(t+\tau)\right|^{2} d \tau d t .
\end{aligned}
$$

Of course, the integral $\int_{0}^{\infty}$ can be replaced by $\int_{0}^{\delta}$ for any $\delta>0$.

The characterization $(1.15)^{*}$ together with the norm $(1.16)^{*}$ immediately imply the following result: Let $|u|_{H^{s}(\mathbf{R} ; X)}$ be any norm for the spaces $H^{s}(\mathbf{R} ; X), s \geq 0$. Then for $s=k+\beta, k \in \mathbf{N}, \beta \in(0,1)$,

$$
\|u\|_{H^{s}(\mathbf{R} ; X)}=|u|_{H^{k}(\mathbf{R} ; X)}+\left|u^{(k)}\right|_{H^{\beta}(\mathbf{R} ; X)}
$$

defines an equivalent norm. 
As a final remark in this introductory subsection on fractional order Sobolev spaces we note that $C_{0}^{\infty}\left(\mathbf{R} ; \mathbf{R}^{n}\right)$ is dense in $H^{s}\left(\mathbf{R} ; \mathbf{R}^{n}\right)$ for any $s \geq 0[11$, p. 31].

1.2.3. Continuity of the shift in $H^{s}(\mathbf{R} ; X)$. Analogous to integer order Sobolev spaces we have the following result.

Proposition 1.1. For every $s \geq 0$ and $u \in H^{s}(\mathbf{R} ; X)$ we have

$$
\lim _{\tau \rightarrow 0} u(\tau+\cdot)=u(\cdot) \quad \text { in } H^{s}(\mathbf{R} ; X) .
$$

PROOF. Using the norm (1.11) we obtain

$$
\begin{aligned}
\|\| u(\tau+\cdot)-u(\cdot)||_{H^{s}(\mathbf{R} ; X)}^{2} & =\int_{-\infty}^{\infty}\left(1+|y|^{2}\right)^{s}|\hat{u}(y)|^{2}\left|e^{i y \tau}-1\right|^{2} d y \\
& =2 \int_{-\infty}^{\infty}(1-\cos y \tau)\left(1+|y|^{2}\right)^{s}|\hat{u}(y)|^{2} d y \rightarrow 0
\end{aligned}
$$

as $\tau \rightarrow 0$ by Lebesgue's dominated convergence theorem.

1.2.4. Continuous embeddings. The following embedding results will be used frequently.

Proposition 1.2. Let $s>\frac{1}{2}$. Then

$$
H^{s}(\mathbf{R} ; X) \subset\left\{u \in C(\mathbf{R} ; X) \mid \lim _{t \rightarrow \pm \infty} u(t)=0\right\}
$$

the embedding being continuous with respect to the $H^{s}(\mathbf{R} ; X)$ and the supremum norm.

Proof. Let $u \in H^{s}(\mathbf{R} ; X)$ and let $\hat{u}=\mathcal{F} u$. Then

$$
\begin{aligned}
\int_{-\infty}^{\infty}|\hat{u}(y)| d y & \leq\left(\int_{-\infty}^{\infty}\left(1+y^{2}\right)^{-s} d y\right)^{1 / 2}\left(\int_{-\infty}^{\infty}\left(1+y^{2}\right)^{s}|\hat{u}(y)|^{2} d y\right)^{1 / 2} \\
& \leq c \mid\|u\|_{H^{s}} .
\end{aligned}
$$

By the Riemann Lebesgue lemma (see for instance [7, p. 401]), which is also true for $X$-valued functions, $u$ is uniformly continuous and $\lim _{t \rightarrow \pm \infty} u(t)=0$. Moreover

$$
|u(t)| \leq \frac{1}{\sqrt{2 \pi}} \int_{-\infty}^{\infty}\left|e^{i y t} \hat{u}(y)\right| d y=\frac{1}{\sqrt{2 \pi}}|\hat{u}|_{L^{1}(\mathbf{R} ; X)} \leq c\left|\left\|u|\||_{H^{s}},\right.\right.
$$

which ends the proof.

Proposition 1.3. Let $0<s_{1}<s_{2}$. Then $H^{s_{2}}(\mathbf{R} ; X) \subset H^{s_{1}}(\mathbf{R} ; X)$ is a continuous embedding.

ProOF. Let $u \in H^{s_{2}}(\mathbf{R} ; X)$ and put $\hat{u}=\{u$. Then

$$
\begin{aligned}
\|\| u \|_{H^{s_{1}}}^{2} & =\int_{-\infty}^{\infty}\left(1+y^{2}\right)^{s_{1}}|\hat{u}(y)|^{2} d y=\int_{-\infty}^{\infty}\left(1+y^{2}\right)^{s_{2}}|\hat{u}(y)|^{2}\left(1+y^{2}\right)^{s_{1}-s_{2}} d y \\
& \leq\|\| u \|_{H^{s_{2}}}^{2} .
\end{aligned}
$$

1.2.5. An extension operator. In order to prove properties for the spaces $H^{s}(I ; X)$, with $I$ a bounded interval, we shall make use of an extension operator. Let $m \in \mathbf{N}$ 
and $I=(a, b),-\infty<a<b<\infty$. Furthermore let $\alpha_{1}, \alpha_{2}$ be $C^{\infty}$-functions on $\mathbf{R}$ such that

$$
\alpha_{1}(\tau) \geq 0, \quad \alpha_{2}(\tau) \geq 0, \quad \alpha_{1}(\tau)+\alpha_{2}(\tau)=1 \quad \text { for } \tau \in \mathbf{R}
$$

and

$$
\alpha_{1}(\tau)=1 \quad \text { for } \tau \leq a, \quad \alpha_{2}(\tau)=1 \quad \text { for } \tau \geq b
$$

Furthermore define the numbers $\beta_{1}, \ldots, \beta_{m}$ by

$$
\sum_{j=1}^{m}(-1)^{\nu} j^{\nu} \beta_{j}=1 \quad \text { for } \nu=0, \ldots, m-1
$$

Note that the $\beta_{j}$ are uniquely determined. For $u \in L^{2}(I ; X)$ and $k=0, \ldots, m-1$ we define $p_{k} u$ by

$$
\left(p_{k} u\right)(\tau)= \begin{cases}\sum_{j=1}^{m}(-j)^{k} \beta_{j} \alpha_{1}(a+j(a-\tau)) u(a+j(a-\tau)) & \text { for } \tau<a, \\ u(\tau) & \text { for } a \leq \tau \leq b, \\ \sum_{j=1}^{m}(-j)^{k} \beta_{j} \alpha_{2}(b-j(\tau-b)) u(b-j(\tau-b)) & \text { for } \tau<b,\end{cases}
$$

compare [11, pp. 14, 38]. Using (1.20)-(1.22) an easy calculation shows

$$
\begin{array}{ll}
\left(p_{k} u\right)^{(\nu)}(a-0)=u^{(\nu)}(a+0), & \nu=0, \ldots, m-k-1 \\
\left(p_{k} u\right)^{(\nu)}(b+0)=u^{(\nu)}(b-0), & \nu=0, \ldots, m-k-1
\end{array}
$$

provided $u^{(\nu)}(a+0)$, respectively $u^{(\nu)}(b-0)$, exist. Moreover we see that $\left(p_{k} u\right)^{(\nu)}(\tau)$ $=0$ for $|\tau|$ sufficiently large. Therefore

$$
p_{k} u \in H^{\nu}(\mathbf{R} ; X) \quad \text { for } u \in H^{\nu}(I ; X), \nu=0, \ldots, m-k
$$

Let $u \in H^{\nu}(I ; X), \nu \leq m-k$. Then for $\mu=0, \ldots, \nu$

$$
\begin{aligned}
\left|\left(p_{k} u\right)^{(\mu)}\right|_{L^{2}(\mathbf{R} ; X)}^{2} & =\int_{-\infty}^{a}\left|\sum_{j=1}^{m} \beta_{j}(-j)^{k+\mu}\left(\alpha_{1} u\right)^{(\mu)}(a+j(a-\tau))\right|^{2} d \tau \\
& +\int_{a}^{b}\left|u^{(\mu)}(\tau)\right|^{2} d \tau+\int_{b}^{\infty}\left|\sum_{j=1}^{m} \beta_{j}(-j)^{k+\mu}\left(\alpha_{2} u\right)^{(\mu)}(b-j(\tau-b))\right|^{2} d \tau
\end{aligned}
$$


Using Leibnitz' rule we get for the first integral

$$
\begin{aligned}
& \int_{-\infty}^{a}\left|\sum_{j=1}^{m} \beta_{j}(-j)^{k+\mu}\left(\alpha_{1} u\right)^{(\mu)}(a+j(a-\tau))\right|^{2} d \tau \\
& \quad=\int_{-\infty}^{a}\left|\sum_{j=1}^{m} \beta_{j}(-j)^{k+\mu} \sum_{\kappa=0}^{\mu}\left(\begin{array}{c}
\mu \\
\kappa
\end{array}\right) \alpha_{1}^{(\mu-\kappa)}(a+j(a-\tau)) u^{(\kappa)}(a+j(a-\tau))\right|^{2} d \tau \\
& \leq m(\mu+1) \sum_{j=1}^{m} \beta_{j}^{2} j^{2(k+\mu)} \sum_{\kappa=0}^{\mu}\left(\begin{array}{l}
\mu \\
\kappa
\end{array}\right)^{2}\left(\max _{[a, b]}\left|\alpha_{1}^{(\mu-\kappa)}\right|\right)^{2} \\
& \quad \cdot \int_{a-(b-a) / j}^{a}\left|\mu^{(\kappa)}(a+j(a-\tau))\right|^{2} d \tau \\
& \leq c \sum_{j=1}^{m} \sum_{\kappa=0}^{\mu} \int_{a}^{b}\left|u^{(\kappa)}(\tau)\right|^{2} d \tau \leq \tilde{c}|u|_{H^{\mu}(I ; X)}^{2} .
\end{aligned}
$$

An analogous estimate is true for the third integral. Therefore

$$
\left|p_{k} u\right|_{H^{\nu}(\mathbf{R} ; X)} \leq c|u|_{H^{\nu}(I ; X)}, \quad u \in H^{\nu}(I ; X), \nu=0, \ldots, m-k,
$$

where the constant $c$ does not depend on $\nu$ and $k$. We have shown that

$$
p_{k} \in \mathcal{L}\left(H^{\nu}(I ; X), H^{k}(\mathbf{R} ; X)\right), \quad \nu=0, \ldots, m-k ; k=0, \ldots, m-1
$$

(for the meaning of $\mathcal{L}$ see $\S 1.3$ ). By construction of $p_{k}$

$$
p_{k} u \mid I=u \quad \text { for all } u \in L^{2}(I ; X) \text {. }
$$

We call $p_{k}$ an extension operator corresponding to the interval $I$. The restriction operator $r$ is defined by

$$
(r u)(\tau)=u(\tau), \quad \text { for } \tau \in I, u \in L^{2}(\mathbf{R} ; X) .
$$

Obviously

$$
r \in \mathcal{L}\left(H^{k}(\mathbf{R} ; X), H^{k}(I ; X)\right), \quad k=0,1, \ldots
$$

Note, that

$$
(r u)^{(k)}=r u^{(k)}, \quad u \in H^{k}(\mathbf{R} ; X) .
$$

1.2.6. The spaces $H^{s}(I ; X), s \geq 0$. Following $[11$, p. 40] we define

$$
H^{s}(I ; X)=\left[H^{m}(I ; X), L^{2}(I ; X)\right]_{\theta}
$$

where $s=(1-\theta) m, m \in \mathbf{N}, 0<\theta<1$; the norm in $H^{s}(I ; X)$ is denoted by $|\cdot|_{H^{s}(I ; X)}$. Equivalently one could define $H^{s}(I ; X)$ to be the restriction of the elements of $H^{s}(\mathbf{R} ; X)$ to $I$.

Proposition 1.4 (COMPARE [11, TheOREM 1.9.1]). For every $s \geq 0$

$$
H^{s}(I ; X)=\left\{r u \mid u \in H^{s}(\mathbf{R} ; X)\right\} \text {. }
$$

Moreover

$$
\|u\|_{H^{s}(I ; X)}:=\inf _{\substack{v \in H^{s}(\mathbf{R} ; X) \\ r v=u}}|v|_{H^{s}(\mathbf{R} ; X)}
$$

defines an equivalent norm on $H^{s}(I ; X)$. 
Proof. Using (1.12), (1.23) and the definition of $H^{s}(I ; X)$ given at the beginning of this subsection the interpolation theorem [11, Theorem 5.1] implies $p_{0} \in \mathcal{L}\left(H^{s}(I ; X), H^{s}(\mathbf{R} ; X)\right)$ and $r \in \mathcal{L}\left(H^{s}(\mathbf{R} ; X), H^{s}(I ; X)\right)$ for $s \leq m$. For every $u \in H^{s}(I ; X)$ we have $u=r p_{0} u$, so that $u$ is the restriction to $I$ of some function (namely $p_{0} u$ ) in $H^{s}(\mathbf{R} ; X)$. On the other hand $r v \in H^{s}(I ; X)$ for every $v \in H^{s}(\mathbf{R} ; X)$. Equivalence of the norms follows from

$$
\|u\|_{H^{s}(I ; X)} \leq\left|p_{0} u\right|_{H^{s}(\mathbf{R} ; X)} \leq c|u|_{H^{s}(I ; X)}
$$

and

$$
|u|_{H^{s}(I ; X)}=|r v|_{H^{s}(I ; X)} \leq c|v|_{H^{s}(\mathbf{R} ; X)}{ }^{1}
$$

for any $v \in H^{s}(\mathbf{R} ; X)$ with $r v=u$. Here $|\cdot|_{H^{s}(I ; X)}$ denotes the interpolation norm of $H^{s}(I ; X)$. This ends the proof.

Similarly to (1.15) and (1.19) we have the following characterization of the spaces $H^{s}(I ; X)$.

Proposition 1.5. Let $s=k+\beta, k \in \mathbf{N}, 0<\beta<1$. Then

$$
H^{s}(I ; X)=\left\{v \in H^{(k)}(I ; X) \mid v^{(k)} \in H^{\beta}(I ; X)\right\}
$$

and

$$
\|\left.|v|\right|_{H^{s}(I ; X)}=|v|_{H^{k}(I ; X)}+\left|v^{(k)}\right|_{H^{\beta}(I ; X)}
$$

is an equivalent norm.

Proof. Let $v \in H^{s}(I ; \mathbf{R})$, i.e. $v=r u$ with $u \in H^{s}(\mathbf{R} ; X)$ by Proposition 1.4. From $(1.15)^{*}$ we get $u \in H^{k}(\mathbf{R} ; X)$ and $u^{(k)} \in H^{\beta}(\mathbf{R} ; X)$. Therefore $v=r u \in$ $H^{k}(I ; X)$ and $v^{(k)}=r u^{(k)} \in H^{\beta}(I ; X)$ (see (1.19)) again by Proposition 1.4.

Suppose now that $v \in H^{k}(I ; X)$ and $v^{(k)} \in H^{\beta}(I ; X)$ and let the operator $p_{0}$ be defined as in $\S 1.2 .5$ with $m \geq k+1$. Then $p_{0} v \in H^{k}(\mathbf{R} ; X)$ and

$$
\left(p_{0} v\right)^{(k)}=u_{1}+p_{k} v^{(k)},
$$

where

$$
u_{1}(\tau)=\left\{\begin{array}{r}
\sum_{j=1}^{m}(-j)^{k} \beta_{j} \sum_{\kappa=0}^{k-1}\left(\begin{array}{l}
k \\
\kappa
\end{array}\right) \alpha_{1}^{(k-\kappa)}(a+j(a-\tau)) u^{(\kappa)}(a+j(a-\tau)) \\
0 \quad \text { for } a \leq \tau \leq b, \\
\sum_{j=1}^{m}(-j)^{k} \beta_{j} \sum_{\kappa=0}^{k-1}\left(\begin{array}{l}
k \\
\kappa
\end{array}\right) \alpha_{2}^{(k-\kappa)}(b-j(\tau-b)) u^{(\kappa)}(b-j(\tau-b)) \\
\text { for } \tau>b .
\end{array}\right.
$$

Obviously $u_{1} \in H^{1}(\mathbf{R} ; X)$. Since $p_{k} \in \mathcal{L}\left(H^{\nu}(I ; X), H^{\nu}(\mathbf{R} ; X)\right), \nu=0, \ldots, m-k$, the interpolation theorem [11, Theorem 5.1] implies $p_{k} \in \mathcal{L}\left(H^{\beta}(I ; X), H^{\beta}(\mathbf{R} ; X)\right)$. Therefore $\left(p_{0} v\right)^{(k)} \in H^{\beta}(\mathbf{R} ; X)$. This together with $p_{0} v \in H^{k}(\mathbf{R} ; X)$ implies $p v \in$ $H^{s}(\mathbf{R} ; X)$. Then $v=r p_{0} v \in H^{s}(I ; X)$ by Proposition 1.4.

${ }^{1}$ Here and in the rest of $\S 1 c, \tilde{c}, c_{\nu}$ denote constants which need not have the same values on each occasion they occur. 
Let $\|v\|_{H^{s}(I ; X)}$ be the norm defined in (1.27) with $|u|_{H^{s}(\mathbf{R} ; X)}=|u|_{H^{k}}(\mathbf{R} ; X)+$ $\left|u^{(k)}\right|_{H^{\beta}(\mathbf{R} ; X)}$ and choose $u \in H^{s}(\mathbf{R} ; X)$ such that $r u=v$. Then $|v|_{H^{k}(I ; X)} \leq$ $|u|_{H^{k}(\mathbf{R} ; X)}$ and because of (1.19) also

$$
\left\|v^{(k)}\right\|_{H^{\beta}(I ; X)}=\inf _{\substack{w \in H^{\beta}(\mathbf{R} ; X) \\ r w=v^{(k)}}}|w|_{H^{\beta}(\mathbf{R} ; X)} \leq\left|u^{(k)}\right|_{H^{\beta}(\mathbf{R} ; X)} .
$$

Therefore $\||v|\|_{H^{s}(I ; X)} \leq|u|_{H^{s}(\mathbf{R} ; X)}$ for all $u \in H^{s}(\mathbf{R} ; X)$ with $r u=v$. This proves

$$
\|v\|_{H^{s}(I ; X)} \leq\|v\|_{H^{s}(I ; X)} .
$$

On the other hand we have

$$
\begin{aligned}
\|v\|_{H^{s}(I ; X)} & \leq\left|p_{0} v\right|_{H^{s}(\mathbf{R} ; X)}=\left|p_{0} v\right|_{H^{k}(\mathbf{R} ; X)}+\left|\left(p_{0} v\right)^{(k)}\right|_{H^{\beta}(\mathbf{R} ; X)} \\
& \leq c|v|_{H^{k}(\mathbf{R} ; X)}+\left|u_{1}\right|_{H^{\beta}(\mathbf{R} ; X)}+\left|p_{k} v^{(k)}\right|_{H^{\beta}(\mathbf{R} ; X)} \\
& \leq c|v|_{H^{k}(I ; X)}+c_{1}\left|u_{1}\right|_{H^{1}(\mathbf{R} ; X)}+c_{2}\left|v^{(k)}\right|_{H^{\beta}(I ; X)}
\end{aligned}
$$

where we also have used Proposition 1.3. By computations similar to those leading to (1.23) we see that

$$
\left|u_{1}\right|_{H^{1}(\mathbf{R} ; X)} \leq \tilde{c}|v|_{H^{k}(I ; X)}
$$

Altogether we have

$$
\|v\|_{H^{s}(I ; X)} \leq c \mid\|v\| \|_{H^{s}(I ; X)}
$$

where $c>0$ is not dependent on $v \in H^{s}(I ; X)$.

1.2.7. Continuous embeddings for $H^{s}(I ; X)$.

Proposition 1.6. The following embeddings are continuous:

$$
H^{s}(I ; X) \subset C(I ; X), \quad s>\frac{1}{2},
$$

and

$$
H^{s_{2}}(I ; X) \subset H^{s_{1}}(I ; X), \quad 0<s_{1}<s_{2} .
$$

Proof. Let $s>\frac{1}{2}$ and $u \in H^{s}(I ; X)$. Then $p_{0} u \in H^{s}(\mathbf{R} ; X)$ and $p_{0} u$ is continuous. Using boundedness of $p_{0}$ we further have

$$
|u|_{C(I ; X)} \leq \sup _{\mathbf{R}}\left|p_{0} u\right| \leq c\left|p_{0} u\right|_{H^{s}(\mathbf{R} ; X)} \leq \tilde{c}|u|_{H^{s}(I ; X)},
$$

where we used Proposition 1.2 and the fact that $p_{0} \in \mathcal{L}\left(H^{s}(I ; X), H^{s}(\mathbf{R} ; X)\right)$. The proof of the second embedding is analogous.

1.2.8. Inner description of $H^{s}(I ; X)$. We now derive a representation of the spaces $H^{s}(I ; X)$ for $s \geq 0$, which uses only values of $u(\tau)$ for $\tau \in I$. This is called inner description of $H^{s}(I ; X)$. Without loss of generality we assume that $I=(-1,0)$ for the rest of this section.

THEOREM 1.7. Let $s=k+\beta$ with $k \in \mathbf{N}$ and $\beta \in(0,1)$. Then the set of functions u for which

$$
|u|_{H^{k}(I ; X)}^{2}+\int_{0}^{1} t^{-1-2 \beta} \int_{-1}^{-t}\left|u^{(k)}(\tau)-u^{(k)}(t+\tau)\right|^{2} d \tau d t
$$

is finite coincides with $H^{s}(I ; X)$ and $(1.28)$ defines an equivalent norm on $H^{2}(I ; X)$. 
REMARK 1.8. In the general case $I=(a, b)$ the integral in (1.28) has to be replaced by

$$
\int_{0}^{b-a} t^{-1-2 \beta} \int_{a}^{b-t}\left|u^{(k)}(\tau)-u^{(k)}(t+\tau)\right|^{2} d \tau d t
$$

Moreover we can replace the upper bound $b-a$ of the outer integration by any $\delta \in(0, b-a)$.

ProOF OF THEOREM 1.7. By Proposition 1.5 it is sufficient to prove the result for $s \in(0,1)$, i.e. $k=0$ and $s=\beta \in(0,1)$. Let $\|u\|_{H^{\beta}(I: X)}$ be the norm defined in (1.27) where

$$
|v|_{H^{\beta}(\mathbf{R} ; X)}^{2}=|v|_{L^{2}(\mathbf{R} ; X)}^{2}+\int_{0}^{1} t^{-1-2 \beta} \int_{-\infty}^{\infty}|v(\tau)-v(t+\tau)|^{2} d \tau d t .
$$

(See $(1.16)^{*}$ and the remark following it.) Then obviously

$$
|u|_{H^{\beta}(I ; X)} \leq|v|_{H^{\beta}(\mathbf{R} ; X)}
$$

for all $v \in H^{\beta}(\mathbf{R} ; X)$ with $r v=u$. Thus

$$
|u|_{H^{s}(I ; X)} \leq\|u\|_{H^{s}(I ; X)} .
$$

On the other hand

$$
\|u\|_{H^{\beta}(I ; X)} \leq\left|p_{0} u\right|_{H^{\beta}(\mathbf{R} ; X)},
$$

where $p_{0}$ is the extension operator defined in $\S 1.2 .5$ with $m=1$. Since $p_{0} \in$ $\mathcal{L}\left(L^{2}(I ; X), L^{2}(\mathbf{R} ; X)\right)$, it is clear that

$$
\left|p_{0} u\right|_{L^{2}(\mathbf{R} ; X)} \leq c|u|_{L^{2}(I ; X)} .
$$

Therefore it remains to estimate

$$
J=\int_{0}^{1} t^{-1-2 \beta} \int_{-\infty}^{\infty}\left|\left(p_{0} u\right)(\tau)-\left(p_{0} u\right)(t+\tau)\right|^{2} d \tau d t .
$$

In our situation $p_{0}$ has the simple form $p_{0}=q_{1}+q_{2}$, where for $u \in L^{2}(I ; X)$

$$
\left(q_{1} u\right)(\tau)= \begin{cases}\alpha_{1}(\tau) u(\tau) & \text { for } \tau \geq-1, \\ \alpha_{1}(-2-\tau) u(-2-\tau) & \text { for } \tau<-1\end{cases}
$$

and

$$
\left(q_{2} u\right)(\tau)= \begin{cases}\alpha_{2}(-\tau) u(-\tau) & \text { for } \tau>0 \\ \alpha_{2}(\tau) u(\tau) & \text { for } \tau \leq 0\end{cases}
$$

Therefore

$$
\begin{aligned}
J \leq & 2 \int_{0}^{-} t^{-1-2 \beta} \int_{-\infty}^{\infty}\left|\left(q_{1} u\right)(\tau)-\left(q_{1} u\right)(t+\tau)\right|^{2} d \tau d t \\
& +2 \int_{0}^{1} t^{-1-2 \beta} \int_{-\infty}^{\infty}\left|\left(q_{2} u\right)(\tau)-\left(q_{2} u\right)(t+\tau)\right|^{2} d \tau d t \\
= & 2\left(J_{1}+J_{2}\right) .
\end{aligned}
$$


In the following we only give the estimate for $J_{2}$. Taking into account the definition of $q_{2}$ and the fact that $\alpha_{2}(\tau)=0$ for $\tau \leq-1$ we obtain

$$
\begin{aligned}
J_{2}= & \int_{0}^{1} t^{-1-2 \beta} \int_{-1-t}^{-1}\left|\left(\alpha_{2} u\right)(t+\tau)\right|^{2} d \tau d t \\
& +\int_{0}^{1} t^{-1-2 \beta} \int_{-1}^{-t}\left|\left(\alpha_{2} u\right)(\tau)-\left(\alpha_{2} u\right)(t+\tau)\right|^{2} d \tau d t \\
& +\int_{0}^{1} t^{-1-2 \beta} \int_{-t}^{0}\left|\left(\alpha_{2} u\right)(\tau)-\alpha_{2}(-t-\tau) u(-t-\tau)\right|^{2} d \tau d t \\
& +\int_{0}^{1} t^{-1-2 \beta} \int_{1-t}^{1}\left|\alpha_{2}(-\tau) u(-\tau)\right|^{2} d \tau d t \\
= & 2 \int_{0}^{1} t^{-1-2 \beta} \int_{-1}^{t-1}\left|\left(\alpha_{2} u\right)(\tau)\right|^{2} d \tau d t \\
& +\int_{0}^{1} t^{-1-2 \beta} \int_{-1}^{-t}\left|\left(\alpha_{2} u\right)(\tau)-\left(\alpha_{2} u\right)(t+\tau)\right|^{2} d \tau d t \\
& +\int_{0}^{1} t^{-1-2 \beta} \int_{-t}^{0}\left|\left(\alpha_{2} u\right)(\tau)-\left(\alpha_{2} u\right)(-t-\tau)\right|^{2} d \tau d t \\
= & 2 I_{1}+I_{2}+I_{3} .
\end{aligned}
$$

Observing $\alpha_{2}(\tau)=o\left((1+\tau)^{\sigma}\right)$ as $\tau \rightarrow-1$ for any $\sigma>0$ we get for $I_{1}$ the estimate

$$
\begin{aligned}
I_{1} & \leq \int_{0}^{1} t^{-1-2 \beta} \max _{[-1, t-1]}\left|\alpha_{2}(\sigma)\right|^{2} \int_{-1}^{t-1}|u(\sigma)|^{2} d \sigma d t \\
& \leq|u|_{L^{2}(I ; X)}^{2} \int_{0}^{1} t^{-1-2 \beta} \max _{[-1, t-1]}\left|\alpha_{2}(\sigma)\right|^{2} d t \\
& \leq c|u|_{L^{2}(I ; X)}^{2} .
\end{aligned}
$$

The estimate for $I_{2}$ is

$$
\begin{aligned}
I_{2} \leq & 2 \int_{0}^{1} t^{-1-2 \beta} \int_{-1}^{-t}\left|\alpha_{2}(\tau)\right|^{2}|u(\tau)-u(i+\tau)|^{2} d \tau d t \\
& +2 \int_{0}^{1} t^{-1-2 \beta} \int_{-1}^{t}\left|\alpha_{2}(\tau)-\alpha_{2}(t+\tau)\right|^{2}|u(t+\tau)|^{2} d \tau d t \\
\leq & 2 \int_{0}^{1} t^{-1-2 \beta} \int_{-1}^{-t}|u(\tau)-u(t+\tau)|^{2} d \tau d t \\
& +2 \max _{[-1,0]}\left|\alpha_{2}^{\prime}(\tau)\right|^{2}|u|_{L^{2}(I ; X)}^{2} \int_{0}^{1} t^{1-2 \beta} d t \\
\leq & c|u|_{H^{\beta}(I ; X)}^{2} .
\end{aligned}
$$

For $I_{3}$ we have

$$
\begin{aligned}
I_{3} \leq & 2 \int_{0}^{1} t^{-1-2 \beta} \int_{-t}^{0}\left|\alpha_{2}(\tau)-\alpha_{2}(-t-\tau)\right|^{2}|u(\tau)|^{2} d \tau d t \\
& +2 \int_{0}^{1} t^{-1-2 \beta} \int_{-t}^{0}\left|\alpha_{2}(-t-\tau)\right|^{2}|u(\tau)-u(-t-\tau)|^{2} d \tau d t \\
= & : A+B,
\end{aligned}
$$


where (observe $|t+2 \tau| \leq t$ and $-1 \leq-t-\tau \leq 0$ )

$$
\begin{aligned}
A & \leq \max _{[-1,0]}\left|\alpha_{2}^{\prime}(\tau)\right|^{2} \int_{0}^{1} t^{1-2 \beta} \int_{-t}^{0}|u(\tau)|^{2} d \tau d t \\
& \leq \max _{[-1,0]}\left|\alpha_{2}^{\prime}(\tau)\right|^{2}|u|_{L^{2}(I ; X)}^{2} \int_{0}^{1} t^{1-2 \beta} d t \\
& =c|u|_{L^{2}(I ; X) .}^{2}
\end{aligned}
$$

The estimate for $B$ is

$$
\begin{aligned}
B & \leq 2 \int_{0}^{1} t^{-1-2 \beta} \int_{-t}^{0}|u(\tau)-u(-t-\tau)|^{2} d \tau d t \\
& =2 \int_{-1}^{0} \int_{-1-\sigma}^{0} \frac{|u(\tau)-u(\sigma)|^{2}}{(-\sigma-\tau)^{1+2 \beta}} d \tau d \sigma \\
& \leq 2 \int_{-1}^{0} \int_{-1-\sigma}^{0} \frac{|u(\tau)-u(\sigma)|^{2}}{|\tau-\sigma|^{1+2 \beta}} d \tau d \sigma \\
& \leq 2 \int_{-1}^{0} \int_{-1}^{0} \frac{|u(\tau)-u(\sigma)|^{2}}{|\tau-\sigma|^{1+2 \beta}} d \tau d \sigma,
\end{aligned}
$$

where we have used $-\sigma-\tau=|\tau|+|\sigma| \geq|\tau-\sigma|$. Using symmetry of the integrand in the last integral with respect to the line $\tau=\sigma$ we obtain

$$
\begin{aligned}
B & \leq 4 \int_{-1}^{0} \int_{\sigma}^{0} \frac{|u(\tau)-u(\sigma)|^{2}}{|\tau-\sigma|^{1+2 \beta}} d \tau d \sigma \\
& =4 \int_{0}^{1} t^{-1-2 \beta} \int_{t-1}^{0}|u(\tau)-u(\tau-t)|^{2} d \tau d t \\
& =4 \int_{0}^{1} t^{-1-2 \beta} \int_{-1}^{-t}|u(\tau)-u(t+\tau)|^{2} d \tau d t \\
& \leq 4|u|_{H^{\beta}(I ; X)}^{2} .
\end{aligned}
$$

Putting all estimates together leads to

$$
J_{2} \leq c|u|_{H^{\beta}(I ; X)}^{2} .
$$

Since $J_{1}$ is estimated analogously, we have $J \leq c|u|_{H^{\beta}(I ; X)}^{2}$ and together with (1.30), (1.31) also

$$
\|u\|_{H^{\beta}(I ; X)} \leq c|u|_{H^{\beta}(I ; X)} .
$$

REMARK 1.9. An alternate proof for Theorem 1.7 can be given by invoking the Theorem of Reduction. Since it relies on Lemma 1.12 below, we preferred the approach using the projection operators.

1.2.9. Another equivalent norm using the modulus of continuity. We give an alternate semigroup characterization of $H^{s}$. As in (1.15), (1.16) let $A$ be the infinitesimal generator of a uniformly bounded $C_{0}$-semigroup $T(t)$ with $D\left(A^{m}\right)=H^{m}(\mathbf{R} ; X)$ for some $m \in \mathbf{N}$. We first consider the case $H^{s}(\mathbf{R} ; X), s<m$.

Proposition 1.10. Let $s=k+\beta$ with $k \in \mathbf{N}$ and $\beta \in(0,1)$. Then the set of functions $u$ for which

$$
|u|_{D\left(A^{k}\right)}^{2}+\int_{0}^{\infty} t^{-1-2 \beta} \sup _{0 \leq h \leq t}\left|(T(h)-I) A^{k} u\right|_{L^{2}(\mathbf{R} ; X)}^{2} d t
$$


is finite, coincides with $H^{s}(\mathbf{R} ; X)$. Moreover (1.31) defines an equivalent norm on $H^{s}(\mathbf{R} ; X)$.

PROOF. Recall the definition of $\|u\|_{H^{s}(\mathbf{R} ; X)}$ in (1.16). Clearly $\|u\|_{H^{s}(\mathbf{R} ; X)}$ is bounded above by the norm in (1.32). It thus suffices to prove

$$
J \leq c \int_{0}^{\infty} t^{-1-2 \beta}\left|(T(t)-I) A^{k} u\right|_{L^{2}(\mathbf{R} ; X)}^{2} d t,
$$

where $c$ is independent of $u \in H^{s}(\mathbf{R} ; X)$ and

$$
J=\int_{0}^{\infty} t^{-1-2 \beta} \sup _{0 \leq h \leq t}\left|(T(h)-I) A^{k} u\right|_{L^{2}(\mathbf{R} ; X)}^{2} d t .
$$

Let $v=A^{k} u$ and observe that

$$
\begin{aligned}
J \leq & 2 \int_{0}^{\infty} t^{-1-2 \beta} \sup _{0 \leq h \leq t}\left|\frac{1}{t}(T(h)-I) \int_{0}^{t}(T(\tau)-I) v d \tau\right|_{L^{2}(\mathbf{R} ; X)}^{2} d t \\
& +2 \int_{0}^{\infty} t^{-1-2 \beta} \sup _{0 \leq h \leq t}\left|\frac{1}{t}(T(h)-I) \int_{0}^{t} T(\tau) v d \tau\right|_{L^{2}(\mathbf{R} ; X)}^{2} d t
\end{aligned}
$$

Using

$$
\begin{aligned}
\frac{1}{t}(T(h)-I) \int_{0}^{t} T(\tau) v d \tau & =\frac{1}{t} A \int_{0}^{h} T(\sigma) \int_{0}^{t} T(\tau) v d \tau d \sigma \\
& =\frac{1}{t} \int_{0}^{h} T(\sigma) A \int_{0}^{t} T(\tau) v d \tau d \sigma \\
& =\frac{1}{t} \int_{0}^{h} T(\sigma) d \sigma(T(t)-I) v
\end{aligned}
$$

we get

$$
\begin{aligned}
\sup _{0 \leq h \leq t} & \left|\frac{1}{t}(T(h)-I) \int_{0}^{t} T(\tau) v d \tau\right|_{L^{2}(\mathbf{R} ; X)}^{2} \\
& \leq \sup _{0 \leq h \leq t} \frac{1}{t^{2}}\left(\int_{0}^{h}\|T(\sigma)\| d \sigma\right)^{2}|(T(t)-I) v|_{L^{2}(\mathbf{R} ; X)}^{2} \\
& \leq M^{2}|(T(t)-I) v|_{L^{2}(\mathbf{R} ; X)}^{2},
\end{aligned}
$$

where $M$ is the uniform bound for $T(t)$. The second integral in (1.34) satisfies

$$
\begin{aligned}
\int_{0}^{\infty} t^{-1-2 \beta} \sup _{0 \leq h \leq t}\left|\frac{1}{t}(T(h)-I) \int_{0}^{t}(T(\tau)-I) v d \tau\right|_{L^{2}(\mathbf{R} ; X)}^{2} d t \\
\quad \leq(M+1)^{2} \int_{0}^{\infty} t^{-1-2 \beta} \frac{1}{t^{2}}\left(\int_{0}^{t}|(T(\tau)-I) v|_{L^{2}(\mathbf{R} ; X)} d \tau\right)^{2} d t \\
\quad \leq(M+1)^{2} \int_{0}^{\infty} t^{-2-2 \beta} \int_{0}^{t}|(T(\tau)-I) v|_{L^{2}(\mathbf{R} ; X)}^{2} d \tau d t \\
\quad=\frac{(M+1)^{2}}{1+2 \beta} \int_{0}^{\infty} \tau^{-1-2 \beta}|(T(\tau)-I) v|_{L^{2}(\mathbf{R} ; X)}^{2} d \tau .
\end{aligned}
$$

The estimates (1.34)-(1.36) imply (1.33) and the claim is verified. 
If $T(t)$ is the shift semigroup then

$$
\omega(t, v)=\sup _{0 \leq h \leq t}|T(h) v-v|_{L^{2}(\mathbf{R} ; X)}
$$

is called the modulus of continuity of $v$. In this case (1.32) becomes

$$
|u|_{H^{k}(\mathbf{R} ; X)}^{2}+\int_{0}^{\infty} t^{-1-2 \beta} \omega\left(t, u^{(k)}\right)^{2} d t
$$

and gives another equivalent norm for $H^{s}(\mathbf{R} ; X)$. Again the upper bound $\infty$ in the integral can be replaced by any $\delta>0$. For the spaces $H^{s}(I ; X)$ we have

Proposition 1.11. Let $s=k+\beta$ with $k \in \mathbf{N}$ and $\beta \in(0,1)$. Then the set of functions $u$ for which

$$
|u|_{H^{k}(I ; X)}^{2}+\int_{0}^{1} t^{-1-2 \beta} \sup _{0 \leq h \leq t} \int_{-1}^{-h}\left|u^{(k)}(h+\tau)-u^{(k)}(\tau)\right|^{2} d \tau d t
$$

is finite, coincides with $H^{s}(I ; X)$. Moreover (1.38) defines an equivalent norm on $H^{s}(I ; X)$.

PROOF. Let $\|u\|_{H^{s}(I ; X)}$ be the norm defined in (1.27), using the norm $|u|_{H^{s}(\mathbf{R} ; X)}$ in $(1.37)$ for $H^{s}(\mathbf{R} ; X)$. Furthermore, recall the norm $\|u\|_{H^{s}(\mathbf{R} ; X)}$ defined in $(1.16)^{*}$ and let \|\|$u \|_{H^{s}(I ; X)}$ be the norm in (1.28) and $|u|_{H^{s}(I ; X)}$ the norm of the theorem. Then for $u \in H^{s}(I ; X)$ we have

$$
\begin{aligned}
\|u\|_{H^{s}(I ; X)} & \leq\left|p_{0} u\right|_{H^{s}(\mathbf{R} ; X)} \leq c\left\|p_{0} u\right\|_{H^{s}(\mathbf{R} ; X)} \\
& \leq \tilde{c}\|\| u\|\|_{H^{s}(I ; X)} \leq \tilde{c}|u|_{H^{s}(I ; X)} .
\end{aligned}
$$

Here we have used $p_{0} \in \mathcal{L}\left(H^{s}(I ; X), H^{s}(\mathbf{R} ; X)\right)$. The inequality

$$
|u|_{H^{s}(I ; X)} \leq\|\| u\|\|_{H^{s}(I ; X)}
$$

is obvious and the result is proved.

1.2.10. Concatenation of functions. Let $-\infty<a<d<b<\infty$ and choose an integer $m \geq 0$. Then it is obvious that for $v \in H^{m}(a, d ; X)$ and $w \in H^{m}(d, b ; X)$ such that $v^{(k)}(d)=w^{(k)}(d), k=0, \ldots, m-1$, the function

$$
u(\tau)= \begin{cases}v(\tau) & \text { for } \tau \in[a, d] \\ w(\tau) & \text { for } \tau \in[d, b]\end{cases}
$$

is in $H^{m}(a, b ; X)$. In order to prove an analogous result for real $s \geq 0$ we need the following lemma.

LEMMA 1.12. (a) Let $\alpha \in\left(0, \frac{1}{2}\right)$. Then there exists a constant $c>0$ such that

$$
\int_{0}^{1} t^{-1-2 \alpha} \int_{-t}^{0}|\phi(\tau)|^{2} d \tau d t \leq c|\phi|_{H^{\alpha}(-1,0 ; X)}^{2}
$$

for all $\phi \in H^{\alpha}(-1,0 ; X)$.

(b) Let $\alpha \in\left(\frac{1}{2}, 1\right)$. Then there exists a constant $c>0$ such that

$$
\int_{0}^{1} t^{-1-2 \alpha} \int_{-t}^{0}|\phi(\tau)-\phi(0)|^{2} d \tau d t \leq c|\phi|_{H^{\alpha}(-1,0 ; X)}^{2}
$$

for all $\phi \in H^{\alpha}(-1,0 ; X)$. 
ProOF. Part (a) is a special case of [17, Theorem 4.3.2], whereas (b) does not seem to be readily available in the literature. For the convenience of the reader we give the proof for both cases.

Define

$$
h(t)= \begin{cases}\phi(-t) & \text { for } 0 \leq t \leq 1 \\ 0 & \text { for } t>1\end{cases}
$$

in case $\alpha \in\left(0, \frac{1}{2}\right)$ and

$$
h(t)= \begin{cases}\phi(-t)-\phi(0) & \text { for } 0 \leq t \leq 1 \\ -\phi(0) & \text { for } t>1\end{cases}
$$

in case $\alpha \in\left(\frac{1}{2}, 1\right)$. Then integration by parts gives

$$
\begin{aligned}
\int_{0}^{1} t^{-1-2 \alpha} \int_{0}^{t}|h(\tau)|^{2} d \tau d t=\frac{1}{2 \alpha} \int_{0}^{1} t^{-2 \alpha}|h(t)|^{2} d t \\
\quad+\frac{1}{2 \alpha} \lim _{t \rightarrow 0^{+}} t^{-2 \alpha} \int_{0}^{t}|h(\tau)|^{2} d \tau-\frac{1}{2 \alpha} \int_{0}^{1}|h(\tau)|^{2} d \tau .
\end{aligned}
$$

Since

$$
t^{-2 \alpha} \int_{0}^{t}|h(\tau)|^{2} d \tau \leq \int_{0}^{t} \tau^{-2 \alpha}|h(\tau)|^{2} d \tau,
$$

we get

$$
\lim _{t \rightarrow 0^{+}} t^{-2 \alpha} \int_{0}^{t}|h(\tau)|^{2} d \tau=0
$$

provided $\int_{0}^{1} \tau^{-2 \alpha}|h(\tau)|^{2} d \tau$ exists. In that case

$$
\int_{0}^{1} t^{-1-2 \alpha} \int_{0}^{t}|h(\tau)|^{2} d \tau d t \leq \frac{1}{2 \alpha} \int_{0}^{1} \tau^{-2 \alpha}|h(\tau)|^{2} d \tau .
$$

We give a bound for $\int_{0}^{1} \tau^{-2 \alpha}|h(\tau)|^{2} d \tau$, which will justify (1.40) and verify the lemma. We put

$$
v(t)=h(t)-\frac{1}{t} \int_{0}^{t} h(\tau) d \tau=\frac{1}{t} \int_{0}^{t}[h(t)-h(\tau)] d \tau
$$

for $t>0$ (compare [17, p. 261]). Since $h(t)-h(\tau)=0$ for $t, \tau>1$, we have $v(t)=O(1 / t)$ as $t \rightarrow \infty$. Therefore $v(t) / t=O\left(1 / t^{2}\right)$ as $t \rightarrow \infty$ and $\int_{t}^{\infty}(v(\tau) / \tau) d \tau$ exists for all $t>0$. Then using (1.40) we get

$$
\begin{aligned}
h(t)-v(t)+\int_{t}^{\infty} \frac{v(\tau)}{\tau} d \tau & =\frac{1}{t} \int_{0}^{t} h(\tau) d \tau+\int_{t}^{\infty}\left(\frac{h(\tau)}{\tau}-\frac{1}{\tau^{2}} \int_{0}^{\tau} h(\sigma) d \sigma\right) d \tau \\
& =\frac{1}{t} \int_{0}^{t} h(\tau) d \tau+\int_{t}^{\infty} \frac{d}{d \tau}\left(\frac{1}{\tau} \int_{0}^{\tau} h(\sigma) d \sigma\right) d \tau \\
& =\lim _{t \rightarrow \infty} \frac{1}{t} \int_{0}^{t} h(\tau) d \tau \\
& = \begin{cases}0 & \text { for } \alpha \in\left(0, \frac{1}{2}\right), \\
-\phi(0) & \text { for } \alpha \in\left(\frac{1}{2}, 1\right) .\end{cases}
\end{aligned}
$$


Therefore, for $t>0$,

$$
h(t)= \begin{cases}v(t)-\int_{t}^{\infty} \frac{v(\tau)}{d \tau} & \text { for } \alpha \in\left(0, \frac{1}{2}\right), \\ v(t)-\int_{t}^{\infty} \frac{v(\tau)}{\tau} d \tau-\phi(0) & \text { for } \alpha \in\left(\frac{1}{2}, 1\right) .\end{cases}
$$

In case $\alpha \in\left(\frac{1}{2}, 1\right)$ we have $h(0)=0$ and by continuity of $h$ also $v(0)=0$. Therefore by $(1.42)$

$$
\lim _{t \rightarrow 0^{+}} \int_{t}^{\infty} \frac{v(\tau)}{\tau} d \tau=-\phi(0) \quad \text { in case } \alpha \in\left(\frac{1}{2}, 1\right) .
$$

Using (1.42) we also obtain

$$
\begin{aligned}
& \int_{0}^{\infty} t^{-2 \alpha}|h(t)|^{2} d t \leq 2 \int_{0}^{\infty} t^{-2 \alpha}|v(t)|^{2} d t \\
&+2 \int_{0}^{\infty} t^{-2 \alpha}\left|\int_{\tau}^{\infty} \frac{v(\tau)}{\tau} d \tau\right|^{2} d t, \quad \alpha \in\left(0, \frac{1}{2}\right),
\end{aligned}
$$

and

$$
\begin{aligned}
\int_{0}^{\infty} t^{-2 \alpha}|h(t)|^{2} d t \leq 2 \int_{0}^{\infty} t^{-2 \alpha}|v(t)|^{2} d t \\
+2 \int_{0}^{\infty} t^{-2 \alpha}\left|\int_{t}^{\infty} \frac{v(\tau)}{\tau} d \tau+\phi(0)\right|^{2} d t, \quad \alpha \in\left(\frac{1}{2}, 1\right) .
\end{aligned}
$$

The Hardy inequality as stated in [5, Lemma 3.4.7] (second inequality with $q=2$ and $\alpha_{B B}=(1-2 \alpha) / 2$ if $\alpha \in\left(0, \frac{1}{2}\right)$ and first inequality with $q=2$ and $\alpha_{B B}=(2 \alpha-1) / 2$ if $\left.\alpha \in\left(\frac{1}{2}, 1\right)\right)$ gives

$$
\int_{0}^{\infty} t^{-2 \alpha}\left|\int_{t}^{\infty} \frac{v(\tau)}{\tau} d \tau\right|^{2} d t \leq\left(\frac{2}{2 \alpha-1}\right)^{2} \int_{0}^{\infty} t^{-2 \alpha}|v(t)|^{2} d t, \quad \alpha \in\left(0, \frac{1}{2}\right)
$$

and

$$
\begin{array}{r}
\int_{0}^{\infty} t^{-2 \alpha}\left|\int_{t}^{\infty} \frac{v(\tau)}{\tau} d \tau+\phi(0)\right|^{2} d t \leq\left(\frac{2}{2 \alpha-1}\right)^{2} \int_{0}^{\infty} t^{-2 \alpha}|v(t)|^{2} d t, \\
\alpha \in\left(\frac{1}{2}, 1\right) .
\end{array}
$$

Note, that because of (1.43) we have

$$
\int_{0}^{t} \frac{v(\tau)}{\tau} d \tau=-\phi(0)-\int_{t}^{\infty} \frac{v(\tau)}{\tau} d \tau
$$

in case $\alpha \in\left(\frac{1}{2}, 1\right)$. Therefore

$$
\int_{0}^{\infty} t^{-2 \alpha}|h(t)|^{2} d t \leq \delta \int_{0}^{\infty} t^{-2 \alpha}|v(t)|^{2} d t
$$

where $\delta$ depends on $\alpha$ only. By (1.41) and Cauchy's inequality we get

$$
\int_{0}^{\infty} t^{-2 \alpha}|v(t)|^{2} d t \leq \int_{0}^{\infty} t^{-1-2 \alpha} \int_{0}^{t}|h(t)-h(\tau)|^{2} d \tau d t
$$


Observing that $h(t)=0$ for $t>1$ in case $\alpha \in\left(0, \frac{1}{2}\right)$ we have

$$
\begin{aligned}
\int_{0}^{\infty} & t^{-1-2 \alpha} \int_{0}^{t}|h(t)-h(\tau)|^{2} d \tau d t \\
& =\int_{0}^{1} t^{-1-2 \alpha} \int_{0}^{t}|h(t)-h(\tau)|^{2} d \tau d t+\int_{1}^{\infty} t^{-1-2 \alpha} \int_{0}^{1}|h(\tau)|^{2} d \tau d t \\
& =\int_{0}^{1} t^{-1-2 \alpha} \int_{0}^{t}|h(t)-h(\tau)|^{2} d \tau d t+\frac{1}{2 \alpha} \int_{0}^{1}|h(\tau)|^{2} d \tau .
\end{aligned}
$$

Similarly we get in case $\alpha \in\left(\frac{1}{2}, 1\right)$

$$
\begin{aligned}
\int_{0}^{\infty} t^{-1-2 \alpha} \int_{0}^{t} \mid h(t) & -\left.h(\tau)\right|^{2} d \tau d t=\int_{0}^{1} t^{1-2 \alpha} \int_{0}^{t}|h(t)-h(\tau)|^{2} d \tau d t \\
& +\frac{1}{2 \alpha} \int_{0}^{1}|h(\tau)+\phi(0)|^{2} d \tau .
\end{aligned}
$$

Performing a change of variables in the first integral on the right-hand side of (1.46) (or (1.47)) we obtain

$$
\begin{aligned}
\int_{0}^{1} t^{-1-2 \alpha} \int_{0}^{t}|h(t)-h(\tau)|^{2} d \tau d t=\int_{0}^{1} t^{-1-2 \alpha} \int_{-t}^{0}|\phi(t)-\phi(\tau)|^{2} d \tau d t \\
\quad=\int_{0}^{1} \int_{-1+t}^{0}(t-\tau)^{-1-2 \alpha}|\phi(\tau-t)-\phi(\tau)|^{2} d \tau d t \\
\quad \leq \int_{0}^{1} t^{-1-2 \alpha} \int_{-1+t}^{0}|\phi(\tau-t)-\phi(\tau)|^{2} d \tau d t \\
\quad=\int_{0}^{1} t^{-1-2 \alpha} \int_{-1}^{-t}|\phi(\tau)-\phi(\tau+t)|^{2} d \tau d t .
\end{aligned}
$$

The second integrals in (1.46) and (1.47) obviously equal $\int_{-1}^{0}|\phi(\tau)|^{2} d \tau$. Then from (1.39) and (1.44)-(1.48) we obtain the desired estimates.

Proposition 1.13. Assume that $v \in H^{s}(a, d ; X), w \in H^{s}(d, b ; X)$ where $s=k+\beta, k \in \mathbf{N}, \beta \in(0,1)$ and let $u$ be defined as in (1.39).

(a) If $\beta \in\left(0, \frac{1}{2}\right)$ and $v^{(j)}(d)=w^{(j)}(d), j=0, \ldots, k-1$, then $u \in H^{s}(a, b ; X)$.

(b) If $\beta \in\left(\frac{1}{2}, 1\right)$ and $v^{(j)}(d)=w^{(j)}(d), j=0, \ldots, k$, then $u \in H^{s}(a, b ; X)$.

ProOF. Obviously $u \in H^{k}(a, b ; X)$. It remains to estimate (see Remark 1.8)

$$
\begin{aligned}
J= & \int_{0}^{b-a} t^{-1-2 \beta} \int_{a}^{b-t}\left|u^{(k)}(\tau)-u^{(k)}(t+\tau)\right|^{2} d \tau d t \\
= & \int_{0}^{d-a} t^{-1-2 \beta} \int_{a}^{d-t}\left|v^{(k)}(\tau)-v^{(k)}(t+\tau)\right|^{2} d \tau d t \\
& +\int_{0}^{b-d} t^{-1-2 \beta} \int_{d}^{b-t}\left|w^{(k)}(\tau)-w^{(k)}(t+\tau)\right|^{2} d \tau d t \\
& +\int_{0}^{b-a} t^{-1-2 \beta} \int_{\max (a, d-t)}^{\min (d, b-t)}\left|v^{(k)}(\tau)-w^{(k)}(t+\tau)\right|^{2} d \tau d t \\
= & : J_{1}+J_{2}+J_{3} .
\end{aligned}
$$


Trivially we have $J_{1} \leq\left|v^{(k)}\right|_{H^{\beta}(a, d ; X)}^{2}$ and $J_{2} \leq\left|w^{(k)}\right|_{H^{\beta}(d, b ; X)}^{2}$. For $J_{3}$ we have

$$
\begin{aligned}
J_{3} \leq & 2 \int_{0}^{b-a} t^{-1-2 \beta} \int_{\max (a, d-t)}^{\min (d, b-t)}\left|v^{(k)}(\tau)\right|^{2} d \tau d t \\
& +2 \int_{0}^{b-a} t^{-1-2 \beta} \int_{\max (d, a+t)}^{\min (b, d+t)}\left|w^{(k)}(\tau)\right|^{2} d \tau d t \\
= & : A+B
\end{aligned}
$$

in case $\beta \in\left(0, \frac{1}{2}\right)$ and

$$
\begin{aligned}
J_{3} \leq & 2 \int_{0}^{b-a} t^{-1-2 \beta} \int_{\max (a, d-t)}^{\min (d, b-t)}\left|v^{(k)}(\tau)-v^{(k)}(d)\right|^{2} d \tau d t \\
& +2 \int_{0}^{b-a} t^{-1-2 \beta} \int_{\max (d, a+t)}^{\min (b, d+t)}\left|w^{(k)}(\tau)-w^{(k)}(d)\right|^{2} d \tau d t
\end{aligned}
$$

in case $\beta \in\left(\frac{1}{2}, 1\right)$ where we use $v^{(k)}(d)=w^{(k)}(d)$.

For $A$ we get using Lemma 1.12(a) (with obvious changes of variables in the integrals)

$$
\begin{aligned}
A & \leq \int_{0}^{d-a} t^{-1-2 \beta} \int_{d-t}^{d}\left|v^{(k)}(\tau)\right|^{2} d \tau d t+\int_{d-a}^{b-a} t^{-1-2 \beta} d t \cdot\left|v^{(k)}\right|_{L^{2}(a, d ; X)}^{2} \\
& \leq c\left|v^{(k)}\right|_{H^{\beta}(a, d ; X)}^{2} .
\end{aligned}
$$

Similarly,

$$
\begin{aligned}
B & \leq \int_{0}^{b-d} t^{-1-2 \beta} \int_{d}^{d+t}\left|w^{(k)}(\tau)\right|^{2} d \tau d t+\int_{b-d}^{b-a} t^{-1-2 \beta} d t \cdot\left|w^{(k)}\right|_{L^{2}(d, b ; X)}^{2} \\
& \leq c\left|v^{(k)}\right|_{H^{\beta}(d, b ; X)}^{2} .
\end{aligned}
$$

In case $\beta \in\left(\frac{1}{2}, 1\right)$ we proceed analogously using Lemma 1.12(b).

1.3. Notation. We summarize some additional notation. A list of symbols can be found at the end of this paper.

Throughout $H^{m+\alpha}, m=0,1,2, \ldots, \alpha \in(0,1)$, are the fractional order Sobolev spaces of $\S 1.2$. If $I=(-\infty, 0)$ we employ the weighted function space $L_{g}^{2}=$ $L_{g}^{2}(-\infty, 0 ; X)$, with $|\phi|_{L_{g}^{2}}^{2}=\int_{-\infty}^{0}|\phi(t)|^{2} g(t) d t$. The assumptions on $g$ will guarantee that $L_{g}^{2}$ is a Hilbert space again. Similarly we put $H_{g}^{m}=H_{g}^{m}(-\infty, 0 ; X)$ and $H_{g}^{m+\alpha}=H_{g}^{m+\alpha}(-\infty, 0 ; X)$ (i.e. $d t$ is replaced by $g(t) d t$ in the respective norms). Furthermore we use the product space $Z_{g}=\mathbf{R}^{n} \times L_{g}^{2}$. Frequently we shall drop the notation for the interval $I$ if it is $[s, T]$, so that $H^{m}(X)=H^{m}(s, T ; X)$, for example. Also the notation for $\mathbf{R}^{n}$ as the image space is dropped if no confusion can arise. The set of bounded linear operators between Banach spaces $X$ and $Y$ is denoted by $\mathcal{L}(X, Y)$. Finally the solution of (1.1) with initial time $s$, which is fixed throughout, is denoted by $x(t)=x(t ; \eta, \phi, f, q)$ where $q=\left(A_{0}, \ldots, A_{k}, A, B_{1}, \ldots, B_{k}, B\right)$. 
2. Invariance results. In the following let $q=\left(A_{0}, \ldots, A_{k}, A, B_{1}, \ldots, B_{k}, B\right)$. $q$ will vary in one of the following spaces:

$$
\begin{aligned}
& Q= {\left[\underset{i=0}{\times} L^{2}\left(\mathbf{R}^{n \times n}\right)\right] \times L^{2}\left(L_{1 / g}^{2}\right) \times\left[\underset{i=1}{\times} L^{\infty}\left(\mathbf{R}^{n \times n}\right)\right] \times L^{p}\left(L_{1 / g}^{2}\right), \quad 2<p \leq \infty, } \\
& Q^{*}= {\left[\underset{i=0}{\times} L^{2}\left(\mathbf{R}^{n \times n}\right)\right] \times L^{2}\left(L_{1 / g}^{2}\right) \times\left[\underset{i=1}{\times} L^{\infty}\left(\mathbf{R}^{n \times n}\right)\right] \times L^{2}\left(L_{1 / g}^{2}\right), } \\
& Q_{r}^{*}=\left[\underset{i=0}{\times} L^{2}\left(\mathbf{R}^{n \times n}\right)\right] \times L^{2}\left(L_{1 / g}^{2}\right) \times\left[\underset{i=1}{\times} L^{\infty}\left(\mathbf{R}^{n \times n}\right)\right] \\
& \times\left\{\left.B \in L^{2}\left(L_{1 / g}^{2}\right)|| B\right|_{L^{2}\left(L_{1 / g}^{2}\right)} \leq r\right\}, \quad r>0, \\
& Q_{m}=\left[\underset{i=0}{\times} H^{m-1}\left(\mathbf{R}^{n \times n}\right)\right] \times H^{m-1}\left(L_{1 / g}^{2}\right) \times\left[\underset{i=1}{\times} H^{m}\left(\mathbf{R}^{n \times n}\right)\right] \times H^{m}\left(L_{1 / g}^{2}\right), \\
& m=1,2, \ldots, \text { and }
\end{aligned}
$$

$$
\begin{aligned}
& Q_{m+\alpha}=\left[\underset{i=0}{\stackrel{\times}{\times}} H^{m-1+\alpha}\left(\mathbf{R}^{n \times n}\right)\right] \\
& \times H^{m-1+\alpha}\left(L_{1 / g}^{2}\right) \times\left[\underset{i=1}{\stackrel{k}{\times}} H^{m+\alpha}\left(\mathbf{R}^{n \times n}\right)\right] \times H^{m+\alpha}\left(L_{1 / g}^{2}\right)
\end{aligned}
$$

for $m=1,2, \ldots$ and $\alpha \in(0,1), \alpha \neq \frac{1}{2}$. The $l^{1}$-product-space-norm for $q \in Q$ is denoted by $|q|_{Q}$ and similarly we use $|q|_{Q^{*}},|q|_{Q_{m}}$, and $|q|_{Q_{m+\alpha}}$.

The case $\alpha=\frac{1}{2}$ corresponds to a parameter for which the Hardy inequality is not available. This inequality is used in the proof of Lemma 1.12 below which is basic for the proof of the fundamental Lemma 3.3.

We next list the assumptions on the weighting function $g$ and on the coefficient matrices which will be used in this paper:

(H1) $g \in L_{\text {loc }}^{\infty}(-\infty, 0 ; \mathbf{R}), g>0$ a.e., and there exists a function

$$
G \in L_{\mathrm{loc}}^{\infty}(-\infty, 0 ; \mathbf{R})
$$

such that for almost all $s \in(-\infty, 0]$

$$
g(t+s) \leq G(t) g(s) \quad \text { for all } t \leq 0 .
$$

(H2) For any compact interval $I \subset(-\infty, 0]$ there exists a constant $m=m(I)>0$ such that $g(s) \geq m$ a.e. on $I$.

(H3) $q \in Q$ and $f \in L^{2}\left(\mathbf{R}^{n}\right)$.

$\left(\mathrm{H} 3^{*}\right) q \in Q^{*}$ and $f \in L^{2}\left(\mathbf{R}^{n}\right)$.

$\left(\mathrm{H} 3_{m}\right) q \in Q_{m}$ and $f \in H^{m}\left(\mathbf{R}^{n}\right)$.

$\left(\mathrm{H} 3_{m+\alpha}\right) q \in Q_{m+\alpha}$ and $f \in H^{m+\alpha}\left(\mathbf{R}^{n}\right)$.

Conditions of type (H1) and (H2) occur frequently when infinite delay equations are investigated in state spaces like $\mathbf{R}^{n} \times L_{g}^{2}(\mathrm{cf}$. $[\mathbf{6}, \mathbf{1 0}])$. We shall also use the following notations. 
Assume that $\left(\mathrm{H}^{*}\right)$ holds and let $x:(-\infty, T] \rightarrow \mathbf{R}^{n}$ be such that $x_{t} \in L_{g}^{2}$ for $t \in[s, T]$. Then we put

$$
\begin{aligned}
& L(t) x_{t}=\sum_{i=0}^{k} A_{i}(t) x\left(t-r_{i}\right)+\int_{-\infty}^{0} A(t, \sigma) x(t+\sigma) d \sigma \\
& D(t) x_{t}=x(t)-\sum_{i=1}^{k} B_{i}(t) x\left(t-r_{i}\right)-\int_{-\infty}^{0} B(t, \sigma) x(t+\sigma) d \sigma
\end{aligned}
$$

a.e. on $[s, T]$. Thus (1.1) can be expressed as

$$
\begin{gathered}
D(t) x_{t}=\int_{s}^{t} L(s) x_{s} d s+f(t)+\eta \quad \text { a.e. on }[s, T], \\
x_{s}=\phi .
\end{gathered}
$$

Furthermore, if $q \in H_{g}^{m}$ and $\left(\mathrm{H} 3_{m}\right)$ is satisfied, we put

$$
\begin{aligned}
(D(s) \phi)^{(j)}= & \phi^{(j)}(0)-\sum_{i=1}^{k} \sum_{\nu=0}^{j}\left(\begin{array}{l}
j \\
\nu
\end{array}\right) B_{i}^{(j-\nu)}(s) \phi^{(\nu)}\left(-r_{i}\right) \\
& -\sum_{\nu=0}^{j}\left(\begin{array}{l}
j \\
\nu
\end{array}\right) \int_{-\infty}^{0}\left(\frac{\partial}{\partial t}\right)^{j-\nu} B(s, \sigma) \phi^{(\nu)}(\sigma) d \sigma
\end{aligned}
$$

$j=0, \ldots, m-1$, and

$$
\begin{aligned}
(L(s) \phi)^{(j)}= & \sum_{i=0}^{k} \sum_{\nu=0}^{j}\left(\begin{array}{l}
j \\
\nu
\end{array}\right) A_{i}^{(j-\nu)}(s) \phi^{(\nu)}\left(-r_{i}\right) \\
& +\sum_{\nu=0}^{j}\left(\begin{array}{l}
j \\
\nu
\end{array}\right) \int_{-\infty}^{0}\left(\frac{\partial}{\partial t}\right)^{j-\nu} A(s, \sigma) \phi^{(\nu)}(\sigma) d \sigma
\end{aligned}
$$

$j=0, \ldots, m-2$. If $x:(-\infty, T] \rightarrow \mathbf{R}^{n}$ is such that $x_{t} \in H_{g}^{m}$ for $s \leq t \leq T$ and $x_{s}=\phi$, then

$$
\begin{array}{ll}
(D(s) \phi)^{(j)}=\left.\frac{d^{j}}{d t^{j}} D(t) x_{t}\right|_{t=s}, & j=0, \ldots, m-1, \\
(L(s) \phi)^{(j)}=\left.\frac{d^{j}}{d t^{j}} L(t) x_{t}\right|_{t=s}, & j=0, \ldots, m-2 .
\end{array}
$$

For $x(t)=x(t ; \eta, \phi, f, q), y(t ; \eta, \phi, f, q)$ is given by (1.4). For fixed $q$ we define by

$$
\Phi_{q}(\eta, \phi, f)(t)=\left(y(t ; \eta, \phi, f, q)-f(t), x_{t}(\eta, \phi, f, q)\right), \quad t \in[s, T],
$$

a mapping $\Phi_{q}$ from $Z_{g} \times L^{2}\left(s, T ; \mathbf{R}^{n}\right)$ (or a subspace) into a space of $Z_{g}$-valued functions on $[s, T]$ (to be specified in the statement of our results). Analogously, for fixed $\eta, \phi, f$ we put

$$
\Psi_{\eta, \phi, f}(q)(t)=\left(y(t ; \eta, \phi, f, q)-f(t), x_{t}(\eta, \phi, f, q)\right), \quad t \in[s, T] .
$$

Thus $\Psi_{\eta, \phi, f}$ is a mapping from $Q$ (or a subspace) into a space of $Z_{g}$-valued functions on $[s, T]$.

The fundamental existence and continuous dependence results are given in the following theorem. 
THEOREM 2.1. Let (H1) and (H2) be satisfied.

(a) If in addition $\left(\mathrm{H} 3^{*}\right)$ holds, then for all $(\eta, \phi) \in Z_{g}$, and all $q \in Q^{*}$ there exists a unique solution $x(t)=x(t ; \eta, \phi, f, q)$ of $(1.1)$ on $[s, T]$. For fixed $q \in Q^{*}, \Phi_{q}$ is a continuous linear mapping $Z_{g} \times L^{2}\left(\mathbf{R}^{n}\right) \rightarrow C\left(Z_{g}\right)$. Moreover, $y(t ; \eta, \phi, f, q)-f(t)$ is absolutely continuous and satisfies

$$
\frac{d}{d t}(y(t)-f(t))=L\left(x_{t}\right) \quad \text { a.e. on }[s, T] .
$$

(b) For all bounded subsets $S$ of $Q$ (respectively, all bounded subsets $S^{*}$ of $Q_{r}^{*}$ with $r<1)$ there exists a constant $\beta=\beta(S)\left(\right.$ resp. $\left.\beta=\beta\left(S^{*}\right)\right)$ such that $|\Phi|_{q} \leq \beta$ for all $q \in S$ (resp. $q \in S^{*}$ ).

(c) For all $(\eta, \phi, f) \in Z_{g} \times L^{2}\left(\mathbf{R}^{n}\right), \Psi_{\eta, \phi, f}$ is a Lipschitz-continuous mapping $Q \rightarrow C\left(Z_{g}\right)$, resp., $Q_{1}^{*} \rightarrow C\left(Z_{g}\right)$, i.e. for any bounded subset $S$ of $Q$, resp. $S^{*}$ of $Q_{r}^{*}$ with $r<1$, there exists a constant $l=l(S ; \eta, \phi, f)$, resp. $l=l\left(S^{*} ; \eta, \phi, f\right)$, such that

$$
\left|\Psi_{\eta, \phi, f}\left(q_{1}\right)-\Psi_{\eta, \phi, f}\left(q_{2}\right)\right| \leq l\left|q_{1}-q_{2}\right|_{Q}
$$

for all $q_{1}, q_{2} \in S$, resp. $\leq l\left|q_{1}-q_{2}\right|_{Q^{*}}$ for all $q_{1}, q_{2} \in S^{*}$. Moreover, for any bounded subset $R$ of $Z_{g} \times L^{2}\left(\mathbf{R}^{n}\right)$ there exists a constant $\lambda=\lambda(S ; R)$, resp. $\lambda=\lambda\left(S^{*} ; R\right)$ such that $l(S ; \eta, \phi, f) \leq \lambda(S ; R)$, resp. $l\left(S^{*} ; \eta, \phi, f\right) \leq \lambda\left(S^{*} ; R\right)$, for all $(\eta, \phi, f) \in$ $R$.

An immediate consequence of assertions (b) and (c) is

COROLlaRY 2.2. Let (H1), (H2), and (H3) (resp. $\left(\mathrm{H} 3^{*}\right)$ ) be satisfied. Then $(\eta, \phi, f, q) \rightarrow \Phi_{q}(\eta, \phi, f) \quad\left(\right.$ or equivalently $\left.(\eta, \phi, f, q) \rightarrow \Phi_{\eta, \phi, f}(q)\right)$ defines a Lipschitz-continuous mapping $Z_{g} \times L^{2}\left(\mathbf{R}^{n}\right) \times Q \rightarrow C\left(Z_{g}\right)\left(\right.$ resp. $Z_{g} \times L^{2}\left(\mathbf{R}^{n}\right) \times Q_{r}^{*} \rightarrow$ $C\left(Z_{g}\right)$ for any $\left.r<1\right)$.

For the following invariance results we need some additional notation: Assume that $\left(\mathrm{H} 3_{m}\right)$ holds. For $f \in H^{m}\left(\mathbf{R}^{n}\right)$ let

$$
\mathcal{M}_{1}=\left\{(\eta, \phi) \in Z_{g} \mid \phi \in H_{g}^{1}, D(s) \phi=\eta+f(s)\right\}
$$

and for $m=2,3, \ldots$

$$
\begin{aligned}
\mathcal{M}_{m}=\{( & (\eta, \phi) \in Z_{g} \mid \phi \in H_{g}^{m}, D(s) \phi=\eta+f(s), \\
& \left.(D(s) \phi)^{(j)}=(L(s) \phi)^{(j-1)}+f^{(j)}(s), j=1, \ldots, m-1\right\} .
\end{aligned}
$$

As we shall see, $(\eta, \phi) \in \mathcal{M}_{m}$ will be needed to guarantee that the solution is $(m-1)$-times continuously differentiable at $t=s$. If the dependence of $\mathcal{M}_{m}$ on $f$ is relevant we write $\mathcal{M}_{m}(f)$.

Proposition 2.3. Let (H1), (H2), and $\left(\mathrm{H}^{*}\right)$ hold.

(a) If $f$ is continuous, then $t \rightarrow\left(y(t ; \eta, \phi, f, q), x_{t}(\eta, \phi, f, q)\right)$ is continuous on $[s, T]$. If in addition $\phi$ is continuous, $B_{i} \in C\left(\mathbf{R}^{n \times n}\right), i=1, \ldots, k, B \in C\left(L_{1 / g}^{2}\right)$, and $\eta=D(s) \phi-f(s)$, then also $x(t ; \eta, \phi, f, q)$ is continuous on $(-\infty, T], y(t)=$ $D(t) x_{t}$ for all $t \in[s, T]$ and

$$
\frac{d}{d t}\left(D(t) x_{t}-f(t)\right)=L(t) x_{t} \quad \text { a.e. on }[s, T] .
$$

Furthermore, if also $A_{i} \in C\left(\mathbf{R}^{n \times n}\right), i=0, \ldots, k$, and $A \in C\left(L_{1 / g}^{2}\right)$ then $D(t) x_{t}-$ $f(t)$ is continuously differentiable on $[s, T]$. 
(b) Assume that $\left(\mathrm{H} 3_{m}\right)$ is satisfied and that $(\eta, \phi) \in \mathcal{M}_{m}, m \geq 1$. Then

$$
y(\cdot ; \eta, \phi, f, q) \in H^{m}\left(\mathbf{R}^{n}\right) \quad \text { and } \quad x_{t}(\eta, \phi, f, q) \in H_{g}^{m}\left(-\infty, 0 ; \mathbf{R}^{n}\right)
$$

for all $t \in[s, T]$.

THEOREM 2.4. Let (H1), (H2), and $\left(\mathrm{H} 3_{m}\right)$ be satisfied.

(a) For any $q \in Q_{m}, \Phi_{q}: \mathcal{M}_{m} \times H^{m}\left(\mathbf{R}^{n}\right) \rightarrow C\left(\mathbf{R}^{n} \times H_{g}^{m}\right)$ is a continuous affine mapping.

(b) For all bounded subsets $S$ of $Q_{m}$ there exists a constant $\beta=\beta(S)$ such that $\left|\Phi_{q}\left(\eta, \phi, f_{1}\right)-\Phi_{q}\left(\theta, \psi, f_{2}\right)\right|_{C\left(\mathbf{R}^{n} \times H_{g}^{m}\right)} \leq \beta\left(|\phi-\psi|_{H_{g}^{m}}+\left|f_{1}-f_{2}\right|_{H^{m}}\right)$ for all $q \in S$ and all $(\eta, \phi) \in \mathcal{M}_{m}\left(f_{1}\right),(\theta, \psi) \in \mathcal{M}_{m}\left(f_{2}\right)$.

(c) For all $(\eta, \phi, f) \in \mathcal{M}_{m} \times H^{m}\left(\mathbf{R}^{n}\right), \Psi_{\eta, \phi, f}$ is a Lipschitz-continuous mapping $Q_{m} \rightarrow C\left(\mathbf{R}^{n} \times H_{g}^{m}\right)$, i.e. for every bounded subset $S$ of $Q_{m}$ there exists a constant $l=l(S ; \eta, \phi, f)$ such that

$$
\left|\Psi_{\eta, \phi, f}\left(q_{1}\right)-\Psi_{\eta, \phi, f}\left(q_{2}\right)\right|_{C\left(\mathbf{R}^{n} \times H_{g}^{m}\right)} \leq l\left|q_{1}-q_{2}\right|_{Q_{m}} \quad \text { for all } q_{1}, q_{2} \in S .
$$

Moreover, for every bounded subset $R$ of $\mathcal{M}_{m} \times H^{m}\left(\mathbf{R}^{n}\right)$ there exists a constant $\lambda=\lambda(S ; R)$ such that

$$
l(S ; \eta, \phi, f) \leq \lambda(S ; R) \quad \text { for all }(\eta, \phi, f) \in R .
$$

REMARK. Note that under the assumptions of Theorem $2.4, \mathcal{M}_{m} \times H^{m}\left(\mathbf{R}^{n}\right)$ is a closed affine subspace of $\mathbf{R}^{n} \times H_{g}^{m} \times H^{m}\left(\mathbf{R}^{n}\right)$. If $f^{(j)}(s)=0, j=0, \ldots, m-1$, then $\mathcal{M}_{m} \times H^{m}$ is a closed linear subspace of $\mathbf{R}^{n} \times H_{g}^{m} \times H^{m}\left(\mathbf{R}^{n}\right)$ and $\Phi_{q} \in$ $\mathcal{L}\left(\mathcal{M}_{m} \times H^{m}\left(\mathbf{R}^{n}\right), C\left(\mathbf{R}^{n} \times H_{g}^{m}\right)\right)$.

Assuming $\left(\mathrm{H} 3_{m+\alpha}\right)$ we define for $f \in H^{m+\alpha}\left(\mathbf{R}^{n}\right), m=1,2, \ldots$,

$$
\mathcal{M}_{m+\alpha}=\left\{\begin{array}{l}
\left\{(\eta, \phi) \in \mathcal{M}_{m} \mid \phi \in H_{g}^{m+\alpha}\right\} \quad \text { for } \alpha \in\left(0, \frac{1}{2}\right), \\
\left\{(\eta, \phi) \in \mathcal{M}_{m} \mid \phi \in H_{g}^{m+\alpha},\right. \\
\left.(D(s) \phi)^{(m)}=(L(s) \phi)^{(m-1)}+f^{(m)}(s)\right\} \quad \text { for } \alpha \in\left(\frac{1}{2}, 1\right) .
\end{array}\right.
$$

THEOREM 2.5. Let (H1), (H2), and (H3 $\left.{ }_{m+\alpha}\right), \alpha \in(0,1), \alpha \neq \frac{1}{2}$, hold.

(a) For every $q \in Q_{m+\alpha}, \Phi_{q}: \mathcal{M}_{m+\alpha} \times H^{m+\alpha}\left(\mathbf{R}^{n}\right) \rightarrow C\left(\mathbf{R}^{n} \times H_{g}^{m+\alpha}\right)$ is a continuous affine mapping.

(b) For all bounded subsets $S$ of $Q_{m+\alpha}$ there exists a constant $\beta=\beta(S)$ such that

$$
\left|\Phi_{q}\left(\eta, \phi, f_{1}\right)-\Phi_{q}\left(\theta, \psi, f_{2}\right)\right|_{C\left(\mathbf{R}^{n} \times H_{g}^{m+\alpha}\right)} \leq \beta\left(|\phi-\psi|_{H_{g}^{m+\alpha}}+\left|f_{1}-f_{2}\right|_{H^{m+\alpha}}\right)
$$

for all $q \in S$ and all $(\eta, \phi) \in \mathcal{M}_{m+\alpha}\left(f_{1}\right),(\theta, \psi) \in \mathcal{M}_{m+\alpha}\left(f_{2}\right)$.

(c) For all $(\eta, \phi, f) \in \mathcal{M}_{m+\alpha} \times H^{m+\alpha}\left(\mathbf{R}^{n}\right)$ and every bounded subset $S \subset Q_{m+\alpha}$ there exists a constant $l=l(S ; \eta, \phi, f)$ such that

$$
\left|\Psi_{\eta, \phi, f}\left(q_{1}\right)-\Psi_{\eta, \phi, f}\left(q_{2}\right)\right|_{C\left(\mathbf{R}^{n} \times H_{g}^{m+\alpha}\right)} \leq l\left|q_{1}-q_{2}\right|_{Q_{m+\alpha}} \quad \text { for all } q_{1}, q_{2} \in S .
$$

Moreover, for every bounded subset $R$ of $\mathcal{M}_{m+\alpha} \times H^{m+\alpha}\left(\mathbf{R}^{n}\right)$ there exists a constant $\lambda=\lambda(S ; R)$ such that

$$
l(S ; \eta, \phi, f) \leq \lambda(S ; R) \quad \text { for all }(\eta, \phi, f) \in R .
$$

REMARK. As an immediate consequence from Theorems 2.4 and 2.5 we get that, under the assumptions of these theorems, $\left(y(t ; \eta, \phi, f, q), x_{t}(\eta, \phi, f, q)\right) \in \mathcal{M}_{m}(t)$, 
respectively $\in \mathcal{M}_{m+\alpha}(t)$ for all $t \in[s, T]$, where $\mathcal{M}_{m}(t)$ and $\mathcal{M}_{m+\alpha}(t)$ are defined like $\mathcal{M}_{m}$ and $\mathcal{M}_{m+\alpha}$ with $s$ replaced by $t$.

A special type of equation contained in (1.1) is given by

$$
x(t)=\int_{0}^{t} B(t-\sigma) x(\sigma) d \sigma+\int_{0}^{t} \int_{0}^{\tau} A(\tau-\sigma) x(\sigma) d \sigma d \tau+f(t)
$$

a.e. on $[0, T]$ or, equivalently by

$$
\begin{aligned}
& y(t)=\int_{0}^{t} \int_{0}^{\tau} A(\tau-\sigma) x(\sigma) d \sigma d \tau+f(t), \\
& x(t)=y(t)+\int_{0}^{t} B(t-\sigma) x(\sigma) d \sigma,
\end{aligned}
$$

a.e. on $[0, T]$.

Equation (2.1) contains as special cases Volterra integral- and intergrodifferential equations of convolution type. There are two possibilities to subsume equation (2.1) under (1.1). The first one is to assume that $A(-\sigma)$ and $B(-\sigma)$ are in $L_{1 / g}^{2}\left(-\infty, 0 ; \mathbf{R}^{n \times n}\right)$ and to take $\phi=0$. Then $(2.1)$ is equivalent to

$$
x(t)=\int_{-\infty}^{0} B(-\sigma) x(t+\sigma) d \sigma+\int_{0}^{t} \int_{-\infty}^{0} A(-\sigma) x(\tau+\sigma) d \sigma d \tau+f(t)
$$

a.e. on $[0, T]$,

$$
x(t)=0 \quad \text { for } t<0 .
$$

The advantage is that the homogeneous equation (i.e. $f \equiv$ const) is autonomous. But if one is interested in regularity properties of solutions the conditions on Proposition 2.3 for example are more restrictive than necessary. Note, that the condition $(0,0) \in \mathcal{M}_{m}(f)$ is an additional condition of $f$.

The second possibility to consider (2.1) as a special case of (1.1) is to take any $\phi \in L_{g}^{2}\left(-\infty, 0 ; \mathbf{R}^{n}\right), g$ being any function satisfying (H1), (H2) and to define

$$
\begin{aligned}
& A(\tau, \sigma)= \begin{cases}A(-\sigma) & \text { for }-\tau \leq \sigma \leq 0, \\
0 & \text { for } \sigma<-\tau .\end{cases} \\
& B(\tau, \sigma)= \begin{cases}B(-\sigma) & \text { for }-\tau \leq \sigma \leq 0, \\
0 & \text { for } \sigma<-\tau\end{cases}
\end{aligned}
$$

Then (2.1) is equivalent to

$$
x(t)=\int_{-\infty}^{0} B(t, \sigma) x(t+\sigma) d \sigma+\int_{0}^{t} \int_{-\infty}^{0} A(\tau, \sigma) x(\tau+\sigma) d \sigma d \tau+f(t),
$$

a.e. on $[0, T]$,

$$
x(t)=\phi(t) \quad \text { a.e. on } t<0 .
$$

However, for this equation Proposition 2.3, for example, is not applicable because $A(\tau, \sigma), B(\tau, \sigma)$ are not in $H^{m}\left(0, T ; L_{1 / g}^{2}\right), m \geq 1$, even if $A$ and $B$ are sufficiently smooth.

Therefore it is useful to take the special structure of equation (2.1) into account and to prove the regularity results separately. In analogy to the general case we put $q=(A, B)$. The solution of $(2.2)$ is denoted by $x(t)=x(t ; f, q), y(t)=y(t ; f, q), t \geq$ 0 . Let $T>0$ be given. For fixed $q, f \rightarrow(y(\cdot ; f, q), x(\cdot ; f, q))$ defines a mapping $\Phi_{q}$, whereas for fixed $f$ the mapping $\Psi_{f}$ is defined by $q \rightarrow(y(\cdot ; f, q), x(\cdot ; f, q))$. 
THEOREM 2.6. (a) Let $q \in Q:=L^{2}\left(0, T ; \mathbf{R}^{n \times n}\right) \times L^{2}\left(0, T ; \mathbf{R}^{n \times n}\right)$ and $f \in$ $L^{2}\left(0, T ; \mathbf{R}^{n}\right)$. Then there exists a unique solution $(y(\cdot ; f, q), x(\cdot ; f, q))$ of $(2.2)$ on $[0, T]$ such that

$$
\frac{d}{d t}\left(x(t)-\int_{0}^{t} B(t-\sigma) x(\sigma) d \sigma-f(t)\right)=\int_{0}^{t} A(t-\sigma) x(\sigma) d \sigma
$$

a.e. on $[0, T]$ and $\lim _{t \rightarrow 0^{+}}[x(t)-f(t)]=0$. Moreover, $\Phi_{q}$ is a bounded linear mapping $L^{2}\left(0, T ; \mathbf{R}^{n}\right) \rightarrow L^{2}\left(0, T ; \mathbf{R}^{n}\right) \times L^{2}\left(0, T ; \mathbf{R}^{n}\right)$. For any bounded subset $S$ of $Q$ there exists a constant $\beta=\beta(\mathcal{S})$ such that

$$
\left|\Phi_{q}\right| \leq \beta \quad \text { for all } q \in S .
$$

For all $f \in L^{2}\left(0, T ; \mathbf{R}^{n}\right), \Psi_{f}$ is a Lipschitz-continuous mapping $Q \rightarrow L^{2}\left(0, T ; \mathbf{R}^{n}\right) \times$ $L^{2}\left(0, T ; \mathbf{R}^{n}\right)$, i.e. for any bounded subset $S$ of $Q$ there exists a constant $l=l(S ; f)$ such that

$$
\left|\Psi_{f}\left(q_{1}\right)-\Psi_{f}\left(q_{2}\right)\right|_{L^{2} \times L^{2}} \leq l\left|q_{1}-q_{2}\right|_{Q}
$$

for all $q_{1}, q_{2} \in S$. For any bounded subset $R$ of $L^{2}\left(0, T ; \mathbf{R}^{n}\right)$ there exists a constant $\lambda=\lambda(S ; R)$ such that

$$
l(S ; f) \leq \lambda(S ; R) \quad \text { for all } f \in R .
$$

If in addition $f \in C\left(0, T ; \mathbf{R}^{n}\right)$ then also $x(\cdot ; f, q)$ and $y(\cdot ; f, q)$ are in $C\left(0, T ; \mathbf{R}^{n}\right)$.

(b) Let $q \in Q_{m}:=H^{m-2}\left(0, T ; \mathbf{R}^{n \times n}\right) \times H^{m-1}\left(0, T ; \mathbf{R}^{n \times n}\right), m \geq 1$ an integer ( $H^{-1}$ is understood to be $L^{2}$ here), and $f \in H^{m}\left(0, T ; \mathbf{R}^{n}\right)$. Then $\Phi_{q}$ is a bounded linear mapping $H^{m}\left(0, T ; \mathbf{R}^{n}\right) \rightarrow H^{m}\left(0, T ; \mathbf{R}^{n}\right) \times H^{m}\left(0, T ; \mathbf{R}^{n}\right)$. For any bounded subset $S_{m}$ of $Q_{m}$ there exists a constant $\beta=\beta\left(S_{m}\right)$ such that

$$
\left|\Phi_{q}\right| \leq \beta \quad \text { for all } q \in S_{m} .
$$

For all $f \in H^{m}\left(0, T ; \mathbf{R}^{n}\right), \Psi_{f}$ is a Lipschitz-continuous mapping

$$
Q_{m} \rightarrow H^{m}\left(0, T ; \mathbf{R}^{n}\right) \times H^{m}\left(0, T ; \mathbf{R}^{n}\right) .
$$

Let $l(S ; f)$ be the Lipschitz constant of $\Psi_{f}$ on the bounded subset $S_{m} \subset Q_{m}$. Then for any bounded subset $R_{m}$ of $H^{m}\left(0, T ; \mathbf{R}^{n}\right)$ there exists a constant $\lambda=\lambda\left(S_{m} ; R_{m}\right)$ such that

$$
l\left(S_{m} ; f\right) \leq \lambda\left(S_{m} ; R_{m}\right) \quad \text { for all } f \in R_{m} .
$$

(c) Let $q \in Q_{m+\alpha}:=H^{m-2+\alpha}\left(0, T ; \mathbf{R}^{n \times n}\right) \times H^{m-1+\alpha}\left(0, T ; \mathbf{R}^{n \times n}\right), \alpha \in(0,1)$, $\alpha \neq \frac{1}{2}$, and $f \in H^{m+\alpha}\left(0, T ; \mathbf{R}^{n}\right)$ (here we put

$$
H^{m-2+\alpha}\left(0, T ; \mathbf{R}^{n \times n}\right)=L^{2}\left(0, T ; \mathbf{R}^{n \times n}\right)
$$

for $m=1), m=1,2, \ldots$ Then $\Phi_{q}$ is a bounded linear mapping $H^{m+\alpha}\left(0, T ; \mathbf{R}^{n}\right) \rightarrow$ $H^{m+\alpha}\left(0, T ; \mathbf{R}^{n}\right) \times H^{m+\alpha}\left(0, T ; \mathbf{R}^{n}\right)$. For any bounded subset $S_{m+\alpha}$ of $Q_{m+\alpha}$ there exists a constant $\beta=\beta\left(S_{m+\alpha}\right)$ such that

$$
\left|\Phi_{q}\right| \leq \beta \quad \text { for all } q \in S_{m+\alpha} \text {. }
$$

For all $f \in H^{m+\alpha}\left(0, T ; \mathbf{R}^{n}\right), \Psi_{f}$ is a Lipschitz-continuous mapping

$$
Q_{\alpha} \rightarrow H^{m+\alpha}\left(0, T ; \mathbf{R}^{n}\right) \times H^{m+\alpha}\left(0, T ; \mathbf{R}^{n}\right) .
$$


Let $l\left(S_{m+\alpha} ; f\right)$ be the Lipschitz constant of $\Psi_{f}$ on the bounded set $S_{m+\alpha} \subset Q_{m+\alpha}$. Then for any bounded subset $R_{m+\alpha}$ of $H^{m+\alpha}\left(0, T ; \mathbf{R}^{n}\right)$ there exists a constant $\lambda=\lambda\left(S_{m+\alpha} ; R_{m+\alpha}\right)$ such that

$$
l\left(S_{m+\alpha} ; f\right) \leq \lambda\left(S_{m+\alpha} ; R_{m+\alpha}\right) \quad \text { for all } f \in R_{m+\alpha} .
$$

We shall not give the proof of this theorem. For the convenience of the reader we state the a priori estimates which are analogs to the estimates contained in Lemmas $3.1-3.3$. With these estimates the proof of Theorem 2.6 is quite analogous to those of Theorems 2.1, 2.4 and 2.5 .

Let $\mathcal{U}=\mathcal{U}(A, B, f)$ be the operator defined by

$$
(\mathcal{U}(A, B, f) x)(t)=\int_{0}^{t} B(t-\sigma) x(\sigma) d \sigma+\int_{0}^{t} \int_{0}^{\tau} A(\tau-\sigma) x(\sigma) d \sigma d \tau+f(t)
$$

a.e. on $[0, T]$

for $x \in L^{2}\left(0, T ; \mathbf{R}^{n}\right)$. For the definition of the norms $|\cdot|_{\mu},|\cdot|_{m, \mu},|\cdot|_{m+\alpha, \mu}, \mu<0$, and the convention on the constant $c$ and the function $\rho(\mu)$ see the beginning of the next section.

LEMMA 2.7. (a) Let $f \in L^{2}\left(0, T ; \mathbf{R}^{n}\right)$ and $q \in Q$. Then

$$
|\mathcal{U}(A, B, f) x|_{\mu} \leq|f|_{L^{2}}+\rho(\mu)|q|_{Q}|x|_{\mu}, \quad x \in L^{2}\left(0, T ; \mathbf{R}^{n}\right) .
$$

(b) Let $f \in H^{m}\left(0, T ; \mathbf{R}^{n}\right)$ and $q \in Q_{m}, m \geq 1$ an integer. Then for the unique solution $x=x(\cdot ; f, q)$ of $(2.1)$

$$
|x|_{H^{m}}=|\mathcal{U}(A, B, f) x|_{m, \mu} \leq k\left(|q|_{Q_{m}}\right)|f|_{H^{m}}+\rho(\mu)\left(|A|_{L^{2}}+|B|_{L^{2}}\right)|x|_{m, \mu}
$$

where $k:[0, \infty) \rightarrow \mathbf{R}^{+}$is continuous, $k(0) \geq 1$.

(c) Let $f \in H^{m+\alpha}\left(0, T ; \mathbf{R}^{n}\right)$ and $q \in Q_{m+\alpha}, \alpha \in(0,1), \alpha \neq \frac{1}{2}$. Then for the unique solution $x=x(\cdot ; f, q)$ of $(2.1)$

$$
|x|_{m+\alpha, \mu} \leq c(1+|\mu|)\left(1+|q|_{Q_{m+\alpha}}\right)|f|_{H^{m+\alpha}}+\rho(\mu)|q|_{Q_{m+\alpha}}|x|_{m+\alpha, \mu} .
$$

3. Proofs for the results of $\S 2$. The general idea for the proofs is a standard one, namely to associate with equation (1.1) a fixed point equation in $L^{2}\left(s, T ; \mathbf{R}^{n}\right)$, $H^{m}\left(s, T ; \mathbf{R}^{n}\right)$ or $H^{m+\alpha}\left(s, T ; \mathbf{R}^{n}\right)$, respectively, and to show that the operator corresponding to equation (1.1) is a contraction. Using the standard norms in the spaces mentioned above one would get the desired results on sufficiently small intervals only and had to use stepwise prolongation of solutions in order to get the result on any prescribed interval $[s, T]$. We shall avoid this prolongation procedure by using equivalent weighted norms in the relevant spaces.

Let $\mu<0$ be a real number to be specified later and define the norms

$$
\begin{gathered}
|z|_{\mu}^{2}=\int_{s}^{T} e^{2 \mu(t-s)}|z(t)|^{2} d t, \quad z \in L^{2}(X), \\
|z|_{m, \mu}^{2}=\sum_{j=0}^{m}\left|\frac{d^{j} z}{d t^{j}}\right|_{\mu}^{2}, \quad z \in H^{m}(X), m=1,2, \ldots
\end{gathered}
$$

and

$$
|z|_{m+\alpha, \mu}^{2}=|z|_{m, \mu}^{2}+\left[z^{(m)}\right]_{\alpha, \mu}^{2}, \quad z \in H^{m+\alpha}(X), \alpha \in(0,1)
$$


where

$$
\left[z^{(m)}\right]_{\alpha, \mu}^{2}=\int_{0}^{1} t^{-1-2 \alpha}\left|e^{\mu(\cdot-s)} z^{(m)}(\cdot)-e^{\mu(\cdot-s+t)} z^{(m)}(\cdot+t)\right|_{L^{2}(s, T-t ; X)}^{2} d t
$$

It is trivial that $|\cdot|_{\mu}$ is a norm equivalent to the standard norm on $L^{2}(X)$. Correspondingly $|\cdot|_{m, \mu}$ is an equivalent norm on $H^{m}(X)$. Equivalence of $|\cdot|_{m+\alpha, \mu}$ to $|\cdot|_{m+\alpha, 0}$ is based on the inequalities

$$
\begin{aligned}
& \left|e^{\mu(\cdot-s)} f(\cdot)-e^{\mu(\cdot-s+t)} \dot{f}(\cdot+t)\right|_{L^{2}(s, T-t ; X)}^{2} \\
& \quad \leq 2|f(\cdot)-f(\cdot+t)|_{L^{2}(s, T-t ; X)}^{2}+2|\mu|^{2} t^{2}|f|_{L^{2}(X)}^{2}
\end{aligned}
$$

and

$$
\begin{gathered}
|f(\cdot)-f(\cdot+t)|_{L^{2}(s, T-t ; X)}^{2} \leq 2 e^{2|\mu|(T-s)}\left|e^{\mu(\cdot-s)} f(\cdot)-e^{\mu(\cdot-s+t)} f(\cdot+t)\right|_{L^{2}(s, T-t ; X)}^{2} \\
+2 t^{2} e^{2|\mu|(T-s)}\left(e^{|\mu|}-1\right)^{2}|f|_{\mu}^{2} .
\end{gathered}
$$

Here we used the inequalities $1-e^{\mu t} \leq|\mu| t$ for $t \geq 0$ and $\left(1+e^{|\mu| t}\right)^{2} \leq t^{2}\left(e^{|\mu|}-1\right)^{2}$ for $0 \leq t \leq 1$. Using the norm (3.3) implicitly assumes $T>s+1$. In order to avoid unnecessary complications in the estimates to follow we assume throughout this section that $T>s+r+1$ and $r_{1}>1$. The first assumption obviously is no restriction. Similarly $r_{1}>1$ is no restriction since $\int_{0}^{1}$ can be replaced by $\int_{0}^{\delta}$ with any $\delta>0$ in (3.4) as mentioned in $\S 1.2$.

Let $Y$ be one of the spaces $L^{2}\left(\mathbf{R}^{n}\right), H^{m}\left(\mathbf{R}^{n}\right)$ or $H^{m+\alpha}\left(\mathbf{R}^{n}\right)$, respectively, with the interval $[s, T]$ suppressed in the notation. For $z \in Y$ we put

$$
x(t)= \begin{cases}z(t) & \text { for } s \leq t \leq T, \\ \phi(t-s) & \text { for } t<s .\end{cases}
$$

The operator $U=U(\eta, \phi, f, q)$ is defined by

$$
\begin{aligned}
(U z)(t)= & \eta+f(t)+\sum_{i=1}^{k} B_{i}(t) x\left(t-r_{i}\right)+\int_{-\infty}^{0} B(t, \sigma) x(t+\sigma) d \sigma \\
& +\sum_{i=0}^{k} \int_{s}^{t} A_{i}(\tau) x\left(\tau+r_{i}\right) d \tau+\int_{s}^{t} \int_{-\infty}^{0} A(\tau, \sigma) x(\tau+\sigma) d \sigma d \tau
\end{aligned}
$$

for $z \in Y$ and $t \in[s, T]$.

In the following $c, c_{1}, c_{2}, \ldots$ will be nonnegative constants which may depend on $\alpha, m, p, T-s, g, G$ but not on $\mu, \eta, \phi, f$ or $q$. Similarly, $c(\mu), c_{1}(\mu), \ldots$ and $\rho(\mu)$ are continuous nonnegative functions of $\mu$ not dependent on $\eta, \phi, f$ or $q$. In addition $\rho(\mu)$ will always satisfy $\lim _{\mu \rightarrow-\infty} \rho(\mu)=0$. All these constants and functions need not be the same on each occasion where they appear.

3.1. Proof of TheOREM 2.1 AND Proposition 2.3. In this case $Y=$ $L^{2}\left(\mathbf{R}^{n}\right)$. The fundamental estimate is contained in the following lemma.

LEMMA 3.1. Let (H1), (H2) be satisfied.

(a) Assume $\left(\mathrm{H}^{*}\right)$ and let $(\eta, \phi) \in Z_{g}$. Then

$$
|U(\eta, \phi, f, q) z|_{\mu} \leq c_{1}|\eta|+c_{2}|q|_{Q^{*}}|\phi|_{L_{g}^{2}}+|f|_{L^{2}}+\left(\rho(\mu) \Gamma^{*}+h_{B}(\mu)\right)|z|_{\mu}
$$


for all $z \in Y$, where

$$
\Gamma^{*}=\sum_{i=0}^{k}\left|A_{i}\right|_{L^{2}}+|A|_{L^{2}}+\sum_{i=1}^{k}\left|B_{i}\right|_{L^{\infty}}
$$

and $0 \leq h_{B}(\mu) \leq|B|_{L^{2}}$ with $\lim _{\mu \rightarrow-\infty} h_{B}(\mu)=0$.

(b) If (H3) is satisfied and $(\eta, \phi) \in Z_{g}$, then

$$
|U(\eta, \phi, f, q) z|_{\mu} \leq c_{1}|\eta|+c_{2}|q|_{Q}|\phi|_{L_{g}^{2}}+|f|_{L^{2}}+\rho(\mu)|q|_{Q}|z|_{\mu}
$$

for all $z \in Y$.

PROOF. The result will follow from estimates of the six terms on the right side of (3.6). Obviously

$$
|\eta|_{\mu} \leq(T-s)^{1 / 2}|\eta| \quad \text { and } \quad|f|_{\mu} \leq|f|_{L^{2}}
$$

Moreover

$$
\begin{aligned}
\left|B_{i}(\cdot) x\left(\cdot-r_{i}\right)\right|_{\mu}^{2} & <\left|B_{i}\right|_{L^{\infty}}^{2} \int_{s}^{T} e^{2 \mu(t-s)}\left|x\left(t-r_{i}\right)\right|^{2} d t \\
& \leq\left|B_{i}\right|_{L^{\infty}}^{2}\left(\left(\operatorname{supess}_{[-r, 0]} \frac{1}{g}\right)|\phi|_{L_{g}^{2}}^{2}+e^{2 \mu r_{1}}|z|_{\mu}^{2}\right)
\end{aligned}
$$

and therefore

$$
\left|\sum_{i=1}^{k} B_{i}(\cdot) x\left(\cdot-r_{i}\right)\right|_{\mu} \leq\left(\sum_{i=1}^{k}\left|B_{i}\right|_{L^{\infty}}\right)\left(c|\phi|_{L_{g}^{2}}+\rho(\mu)|z|_{\mu}\right) .
$$

Next we have the estimate

$$
\begin{aligned}
\left|\int_{-\infty}^{0} B(\cdot, \sigma) x(\cdot+\sigma) d \sigma\right|_{\mu}^{2} \leq & 2 \int_{s}^{T}\left(\int_{-\infty}^{0}|B(t, \sigma+s-t) \| \phi(\sigma)| d \sigma\right)^{2} d t \\
& +2 \int_{s}^{T} e^{2 \mu(t-s)}\left(\int_{s}^{t}|B(t, \sigma-t)||z(\sigma)| d \sigma\right)^{2} d t \\
= & \text { I }+ \text { II. }
\end{aligned}
$$

We estimate I and II separately. Using the Cauchy inequality and (H1) we obtain

$$
\begin{aligned}
\mathrm{I} & \leq 2 \int_{s}^{T}\left(\int_{-\infty}^{0} \frac{|B(t, \sigma+s-t)|^{2}}{g(\sigma+s-t)} d \sigma\right)\left(\int_{-\infty}^{0}|\phi(\sigma)|^{2} g(\sigma+s-t) d \sigma\right) d t \\
& \leq 2\left(\operatorname{supess}_{[s-T, 0]}^{[s]}\right)|B|_{L^{2}\left(L_{1 / g}^{2}\right)}^{2}|\phi|_{L_{g}^{2}}^{2} .
\end{aligned}
$$


Using the Cauchy and Hölder inequalities and Minkowski's integral inequality (see for instance [13, p. 14]) we get with $\gamma=\operatorname{supess}_{[s-T, 0]} g$

$$
\begin{aligned}
\mathrm{II} & \leq 2 \int_{s}^{T} e^{2 \mu(t-s)}\left(\int_{s}^{t} \frac{|B(t, \sigma-t)|^{2}}{g(\sigma-t)} d \sigma\right)\left(\int_{s}^{t}|z(\sigma)|^{2} g(\sigma-t) d \sigma\right) d t \\
& \leq 2 \gamma \int_{s}^{T}|B(t, \cdot)|_{L_{1 / g}^{2}}^{2} \int_{s}^{t} e^{2 \mu(t-s)}|z(\sigma)|^{2} d \sigma d t \\
& \leq 2 \gamma|B|_{L^{p}\left(L_{1 / g}^{2}\right)}^{2}\left(\int_{s}^{T}\left(\int_{s}^{t} e^{2 \mu(t-s)}|z(\sigma)|^{2} d \sigma\right)^{p /(p-2)} d t\right)^{(p-2) / p} \\
& \leq 2 \gamma|B|_{L^{p}\left(L_{1 / g}^{2}\right)}^{2} \int_{s}^{T}\left(\int_{\sigma}^{T}\left(e^{2 \mu(t-\sigma)} e^{2 \mu(\sigma-s)}|z(\sigma)|^{2}\right)^{p /(p-2)} d t\right)^{(p-2) / p} d \sigma \\
& \leq 2 \gamma|B|_{L^{p}\left(L_{1 / g}^{2}\right)}^{2}\left(\frac{p-2}{2 p|\mu|}\right)^{(p-2) / p}|z|_{\mu}^{2}
\end{aligned}
$$

and therefore

$$
\left|\int_{-\infty}^{0} B(\cdot, \sigma) x(\cdot+\sigma) d \sigma\right|_{\mu} \leq|B|_{L^{p}\left(L_{1 / g}^{2}\right)}\left(c|\phi|_{L_{g}^{2}}+\rho(\mu)|z|_{\mu}\right)
$$

if (H3) holds. Similarly we find by the dominated convergence theorem

$$
\left|\int_{-\infty}^{0} B(\cdot, \sigma) x(\cdot+\sigma) d \sigma\right|_{\mu} \leq|B|_{L^{2}\left(L_{1 / g}^{2}\right)}\left(c|\phi|_{L_{g}^{2}}+h_{B}(\mu)|z|_{\mu}\right)
$$

if $\left(\mathrm{H}^{*}\right)$ holds. Here the function $h_{B}(\mu)$ has the properties stated in the lemma.

It is easy to obtain the estimate

$$
\begin{aligned}
\left|\int_{s} A_{i}(\tau) x\left(\tau-r_{i}\right) d \tau\right|_{\mu}^{2} \leq & (T-s)\left(\operatorname{supess}_{[-r, 0]} \frac{1}{g}\right)\left|A_{i}\right|_{L^{2}}^{2}|\phi|_{L_{g}^{2}}^{2} \\
& +\frac{1}{|\mu|}\left|A_{i}\right|_{L^{2}}^{2}|z|_{\mu}^{2}, \quad i=0, \ldots, k,
\end{aligned}
$$

and consequently

$$
\left|\sum_{i=0}^{k} \int_{0} A_{i}(\tau) x\left(\tau-r_{i}\right) d \tau\right|_{\mu} \leq\left(\sum_{i=0}^{k}\left|A_{i}\right|_{L^{2}}\right)\left(c|\phi|_{L_{g}^{2}}+\rho(\mu)|z|_{\mu}\right) .
$$

Finally we observe that

$$
\begin{aligned}
\left|\int_{s} \int_{-\infty}^{0} A(\tau, \sigma) x(\tau+\sigma) d \sigma d \tau\right|_{\mu}^{2} \\
\leq 2 \int_{s}^{T}\left(\int_{s}^{t} e^{\mu(t-s)} \int_{-\infty}^{s}|A(\tau, \sigma-\tau)||\phi(\sigma-s)| d \sigma d \tau\right)^{2} d t \\
\quad+2 \int_{s}^{T}\left(\int_{s}^{t} e^{\mu(t-s)} \int_{s}^{\tau}|A(\tau, \sigma-\tau)||z(\sigma)| d \sigma d \tau\right)^{2} d t \\
=: \mathrm{I}+\mathrm{II} .
\end{aligned}
$$


Using the Minkowski and Cauchy inequality we obtain

$$
\begin{aligned}
& \mathrm{I} \leq 2\left(\int_{s}^{T}\left(\int_{\tau}^{T}\left(\int_{-\infty}^{s}|A(\tau, \sigma-\tau) \| \phi(\sigma-s)| d \sigma\right)^{2} d t\right)^{1 / 2} d \tau\right)^{2} \\
& \leq 2\left(\int _ { s } ^ { T } \left(\int_{\tau}^{T}\left(\int_{-\infty}^{s} \frac{|A(\tau, \sigma-\tau)|^{2}}{g(\sigma-\tau)} d \sigma\right)\right.\right. \\
& \left.\left.\times\left(\int_{-\infty}^{s}|\phi(\sigma-s)|^{2} g(\sigma-\tau) d \sigma\right) d t\right)^{1 / 2} d \tau\right)^{2} \\
& \leq 2\left(\operatorname{supess}_{[s-T, 0]} G\right)|\phi|_{L_{1 / g}^{2}}^{2}|A|_{L^{2}\left(L_{1 / g}^{2}\right)}^{2}(T-s)^{2} .
\end{aligned}
$$

The estimate for II is

$$
\begin{aligned}
& \mathrm{II} \leq 2\left(\int _ { s } ^ { T } \left(\int_{\tau}^{T} e^{2 \mu(t-s)}\left(\int_{s}^{T} \frac{|A(\tau, \sigma-\tau)|^{2}}{g(\sigma-\tau)} d \sigma\right)\right.\right. \\
& \left.\left.\leq 2\left(\int_{s}^{T}|z(\sigma)|^{2} g(\sigma-\tau) d \sigma\right) d t\right)^{1 / 2} d \tau\right)^{2} \\
& \left.\quad \cdot\left(\int_{\tau}^{T} \int_{s}^{\tau} e^{2 \mu(t-\sigma)} e^{2 \mu(\sigma-s)}|z(\sigma)|^{2} d \sigma d t\right)^{1 / 2} d \tau\right)^{2} \\
& \leq 2 \gamma\left(\int_{s}^{T}|A(\tau, \cdot)|_{L_{1 / g}^{2}}\left(\int_{s}^{T} e^{2 \mu(\sigma-s)}|z(\sigma)|^{2} \int_{\max (\sigma, \tau)}^{T} e^{2 \mu(t-\sigma)} d t d \sigma\right)^{1 / 2} d \tau\right)^{2} \\
& \leq \gamma \frac{1}{|\mu|}|z|_{\mu}^{2}|A|_{L^{2}\left(L_{1 / g}^{2}\right)}^{2}(T-s) .
\end{aligned}
$$

Summarizing these estimates we arrive at

$$
\left|\int_{s}^{\bullet} \int_{-\infty}^{0} A(\tau, \sigma) x(\tau+\sigma) d \sigma d \tau\right|_{\mu} \leq|A|_{L^{2}\left(L_{1 / g}^{2}\right)}\left(c|\phi|_{L_{g}^{2}}+\rho(\mu)|z|_{\mu}\right) .
$$

The assertion of the lemma now follows from (3.7)--(3.12).

PROOF OF THEOREM 2.1. (a) Let $z_{1}, z_{2} \in L^{2}\left(\mathbf{R}^{n}\right)$. Then according to Lemma 3.1

$$
\begin{aligned}
\left|U(\eta, \phi, f, q) z_{1}-U(\eta, \phi, f, q) z_{2}\right|_{\mu} & =\left|U(0,0,0, q)\left(z_{1}-z_{2}\right)\right|_{\mu} \\
& \leq\left(\rho(\mu) \Gamma^{*}+h_{B}(\mu)\right)\left|z_{1}-z_{2}\right|_{\mu},
\end{aligned}
$$

so that $U$ is a contraction on $L^{2}\left(\mathbf{R}^{n}\right)$ provided that $\mu<0$ is chosen such that

$$
\alpha^{*}=\rho(\mu) \Gamma^{*}+h_{B}(\mu)<1 .
$$

Then there exists a unique fixed point $z \in L^{2}\left(\mathbf{R}^{n}\right)$ of $U$ and the corresponding function $x$ (see (3.5)) is the unique solution of (1.1). 
Next we show that the linear mapping $\Phi_{q}$ has its range in $C\left(Z_{g}\right)$. Using (H1) and the definition of $x(t)$ we get

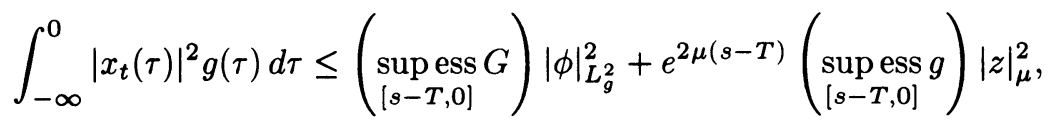

which proves that $\left(y(t)-f(t), x_{t}\right) \in Z_{g}$ for $s \leq t \leq T$. Absolute continuity of $y(t ; \eta, \phi, f, q)-f(t)$ on $[s, T]$ follows from (1.6). In order to establish continuity of $t \rightarrow x_{t}$ we observe that for $M<0$ and $s \leq t_{1}<t_{2} \leq T$ we have

$$
\begin{aligned}
\int_{-\infty}^{0}\left|x_{t_{1}}(\tau)-x_{t_{2}}(\tau)\right|^{2} g(\tau) d \tau \leq & \int_{-\infty}^{M}\left|x_{t_{1}}(\tau)-x_{t_{2}}(\tau)\right|^{2} g(\tau) d \tau \\
& +(\underset{[M, 0]}{\sup \operatorname{ess} g}) \int_{M}^{0}\left|x\left(t_{1}+\tau\right)-x\left(t_{2}+\tau\right)\right|^{2} d \tau \\
= & \mathrm{I}_{1}+\mathrm{I}_{2} .
\end{aligned}
$$

For $\mathrm{I}_{1}$ we get

$$
\begin{aligned}
\mathrm{I}_{1} & \leq 2 \int_{-\infty}^{M+T-s}\left|x_{s}(\tau)\right|^{2} g\left(\tau+s-t_{1}\right) d \tau+2 \int_{-\infty}^{M+T-s}\left|x_{s}(\tau)\right|^{2} g\left(\tau+s-t_{2}\right) d \tau \\
& \leq 4\left(\operatorname{supess}_{[s-T, 0]}\right) \int_{-\infty}^{M+T-s}\left|x_{s}(\tau)\right|^{2} g(\tau) d \tau .
\end{aligned}
$$

This shows that $\mathrm{I}_{1}$ can be made arbitrarily small for all $t_{1}, t_{2} \in[s, T]$ by an appropriate choice of $M$. Then $\mathrm{I}_{2}$ can be made small by choosing $t_{2}-t_{1}$ small.

In order to verify that $\Phi_{q}$ is bounded let $z$ be the fixed point of $u(\eta, \phi, t, q), q \in$ $Q^{*}$. Then from Lemma 3.1 we see that

$$
|z|_{\mu} \leq \frac{1}{1-\alpha^{*}}\left(c_{1}|\eta|+c_{2}|q|_{Q^{*}}|\phi|_{L_{g}^{2}}+|f|_{L^{2}}\right)
$$

with $\alpha^{*}$ as above. A short calculation using (H1) shows that

$$
\left|x_{t}(\eta, \phi, f, q)\right|_{L_{g}^{2}} \leq\left(\operatorname{supess}_{[s-T, 0]}\right)^{1 / 2}|(\eta, \phi)|_{Z_{g}}+\left(\sup _{[s-T, 0]} g\right)^{1 / 2} e^{\mu(s-T)}|z|_{\mu} .
$$

From (3.13) and (3.14) we obtain

$$
\sup _{s \leq t \leq T}\left|x_{t}\right|_{L_{g}^{2}} \leq c|(\eta, \phi)|_{z_{g}}+\frac{c}{1-\alpha^{*}}\left(|\eta|+\left(\Gamma^{*}+|B|_{L^{2}}\right)|\phi|_{L_{g}^{2}}+|f|_{L^{2}}\right) .
$$

Finally by $(\mathrm{H} 2)$ and $\left(\mathrm{H}^{*}\right)$ we have

$$
\begin{aligned}
|y(t)-f(t)| \leq|\eta|+\left\{\left(\sup _{[s-T, 0]} \frac{1}{g}\right)^{1 / 2}\right. & \sum_{i=0}^{k}\left|A_{i}\right|_{L^{2}} \\
& \left.\left.+(T-s)^{1 / 2}|A|_{L^{2}\left(L_{1 / g}^{2}\right)}\right)\right\} \sup _{[s, T]}\left|x_{t}\right|_{L_{g}^{2}} .
\end{aligned}
$$

These estimates imply boundedness of $\Phi_{q}$ for fixed $q \in Q^{*}$. The final assertion of (a) is easily verified. 
(b) If $S^{*}$ is a bounded subset of $Q_{r}^{*}$ with $r<1$, then $\mu$ can be chosen such that

$$
\tilde{\alpha}=\rho(\mu) \sup _{q \in Q_{r}^{*}} \Gamma^{*}+r<1 .
$$

The assertion now follows with (3.14)-(3.16) and $\alpha^{*}$ replaced by $\tilde{\alpha}$ as in (a). Similarly, if $S$ is a bounded subset of $Q$, there exists $\gamma$ such that $|q|_{Q} \leq \gamma$ for all $q \in S$. Then $\mu$ can be chosen such that

$$
\rho(\mu)|q|_{Q} \leq \tilde{\alpha}:=\rho(\mu) \gamma<1, \quad q \in S .
$$

We obtain

$$
|z|_{\mu} \leq \frac{c}{1-\tilde{\alpha}}\left(|\eta|+\gamma|\phi|_{L_{g}^{2}}+|f|_{L^{2}}\right) .
$$

This together with (3.14) and (3.16) ends the proof of (b).

(c) We only verify the estimates for $q \in S, S$ a bounded subset of $Q$, the proof for the other case being quite similar. For $(\eta, \phi, f) \in Z_{g} \times L^{2}\left(\mathbf{R}^{n}\right)$ let $z_{i}$ denote the fixed point of $U\left(\eta, \phi, f, q_{i}\right)$ in $L_{\mu}^{2}, i=1,2$. Using Lemma 3.1 we get

$$
\begin{aligned}
\left|z_{1}-z_{2}\right|_{\mu} & =\left|U\left(\eta, \phi, f, q_{1}\right) z_{1}-U\left(\eta, \phi, f, q_{2}\right) z_{2}\right|_{\mu} \\
& \leq\left|U\left(0,0,0, q_{1}\right)\left(z_{1}-z_{2}\right)\right|_{\mu}+\left|U\left(0, \phi, 0, q_{1}-q_{2}\right) z_{2}\right|_{\mu} \\
& \leq \rho(\mu)\left|q_{1}\right|_{Q}\left|z_{1}-z_{2}\right|+\left(c|\phi|_{L_{g}^{2}}+\rho(\mu)\left|z_{2}\right|_{\mu}\right)\left|q_{1}-q_{2}\right|_{Q} .
\end{aligned}
$$

With $\gamma, \mu$, and $\tilde{\alpha}$ chosen as in (b) this implies

$$
\left|z_{1}-z_{2}\right|_{\mu} \leq c\left(|\phi|_{L_{g}^{2}}+\left|z_{2}\right|_{\mu}\right)\left|q_{1}-q_{2}\right|_{Q}, \quad q_{1}, q_{2} \in \mathcal{S} .
$$

This estimate together with (3.14) gives

$$
\sup _{[s, T]}\left|\left(x_{1}\right)_{t}-\left(x_{2}\right)_{t}\right|_{L_{g}^{2}} \leq c\left(|\phi|_{L_{g}^{2}}+\left|z_{2}\right|_{\mu}\right)\left|q_{1}-q_{2}\right|_{Q} .
$$

Here $x_{i}$ is the solution of (1.1) corresponding to $z_{i}, i=1,2$. Analogously to (3.16) we have

$$
\begin{aligned}
\left|y_{1}(t)-y_{2}(t)\right| \leq & \left(\left(\sup _{[s-T-r, 0]} \frac{1}{g}\right)^{1 / 2}+(T-s)^{1 / 2}\right) \\
& \cdot\left\{\left|q_{1}-q_{2}\right|_{Q} \sup _{[s, T]}\left|\left(x_{2}\right)_{t}\right|_{L_{g}^{2}}+\gamma \sup _{[s, T]}\left|\left(x_{1}\right)_{t}-\left(x_{2}\right)_{t}\right|_{L_{g}^{2}}\right\} .
\end{aligned}
$$

Using (3.17) we obtain

$$
\left|y_{1}(t)-y_{2}(t)\right| \leq c\left(\sup _{[s, T]}\left|\left(x_{2}\right)_{t}\right|_{L_{g}^{2}}+|\phi|_{L_{g}^{2}}+\left|z_{2}\right|_{\mu}\right)\left|q_{1}-q_{2}\right|_{Q}
$$

for $q_{1}, q_{2} \in S$. Inequality (3.14) implies

$$
\sup _{[s, T]}\left|\left(x_{2}\right)_{t}\right|_{L_{g}^{2}} \leq\left(\operatorname{supess}_{[s-T, 0]}\right)^{1 / 2}|(\eta, \phi)|_{Z_{g}}+\left(\operatorname{supess}_{[s-T, 0]}\right)^{1 / 2} e^{\mu(s-T)}\left|z_{2}\right|_{\mu} .
$$

From Lemma 3.1 (b) we further get for $q_{2} \in S$

$$
\left|z_{2}\right|_{\mu} \leq \frac{c}{1-\tilde{\alpha}}\left(|f|_{L^{2}}+|\eta|+\gamma|\phi|_{L_{g}^{2}}\right) .
$$

The estimates (3.17)-(3.20) together prove assertion (c). 
ProOF OF Proposition 2.3. (a) The first assertion is obvious from (1.6). Under the additional assumptions the terms $B_{i}(t) x\left(t-r_{i}\right), i=1, \ldots, k$, are continuous on $\left[s, s+r_{i}\right]$. Continuity of the second term on the right-hand side of (1.1) follows from

$$
\begin{aligned}
& \left|\int_{-\infty}^{0} B\left(t_{1}, \sigma\right) x\left(t_{1}+\sigma\right) d \sigma-\int_{-\infty}^{0} B\left(t_{2}, \sigma\right) x\left(t_{2}+\sigma\right) d \sigma\right| \\
& \quad \leq|B(t, \cdot)|_{L_{1 / g}^{2}}\left|x_{t_{1}}-x_{t_{2}}\right|_{L_{g}^{2}}+\left|B\left(t_{1}, \cdot\right)-B\left(t_{2}, \cdot\right)\right|_{L_{1 / g}^{2}}\left|x_{t_{2}}\right|_{L_{g}^{2}} .
\end{aligned}
$$

Therefore $x$ is continuous on $\left(s, s+r_{1}\right)$. At $t=s$ we have $x(s-0)=\phi(0)$ and $x(s+0)=\phi(0)-D(s) \phi+f(s)+\eta=\phi(0)$ by assumption. Therefore $x$ is continuous at $t=s$ and $B_{i}(t) x\left(t-r_{i}\right)$ is continuous at $t=s+r_{i}$ also. By stepwise continuation we see that $x$ is continuous on $(-\infty, T]$. The proofs for the other statements are easy.

(b) Assume that the result is true for $m-1, m \geq 2$. Then (1.1) implies

$$
\begin{aligned}
& x^{(m-1)}(t)= \sum_{i=1}^{k} B_{i}(t) x^{(m-1)}\left(t-r_{i}\right)+\int_{-\infty}^{0} B(t, \sigma) x^{(m-1)}(t+\sigma) d \sigma \\
&+\frac{d^{m-2}}{d t^{m-2}} L(t) x_{t}+f^{(m-1)}(t)+F_{m-1}(t) \quad \text { a.e. on }[s, T], \\
& x^{(m-1)}(t)=\phi^{(m-1)}(t-s) \quad \text { a.e. on }(-\infty, s),
\end{aligned}
$$

where

$$
\begin{aligned}
F_{j}(t)= & \frac{d}{d t} F_{j-1}(t)+\sum_{i=1}^{k} \dot{B}_{i}(t) x^{(j-1)}\left(t-r_{i}\right) \\
& +\int_{-\infty}^{0} B_{t}(t, \sigma) x^{(j-1)}(t+\sigma) d \sigma, \quad j=1, \ldots, m, \\
& F_{0}(t)=\eta .
\end{aligned}
$$

Condition $\left(\mathrm{H} 3_{m}\right)$ and continuity of $x^{(m-2)}$ imply that $\tilde{f}(t)=\left(d^{m-2} / d t^{m-2}\right) L(t) x_{t}$ $+f^{(m-1)}(t)+F_{m-1}(t)$ is continuous on $[s, T]$. Therefore we can prove continuity of $x^{(m-1)}$ on $(-\infty, T]$ as under (a) above; continuity of $x^{(m-1)}$ at $t=s$ follows from $x^{(m-1)}(s)=\phi^{(m-1)}(0)$ and

$$
\begin{aligned}
x^{(m-1)}(s+0)= & (L(s) \phi)^{(m-2)}+f^{(m-1)}(s)+F_{m-1}(s) \\
& +\sum_{i=1}^{k} B_{i}(s) \phi^{(m-1)}\left(-r_{i}\right)+\int_{-\infty}^{0} B(s, \sigma) \phi^{(m-1)}(\sigma) d \sigma \\
= & (D(s) \phi)^{(m-1)}+\phi^{(m-1)}(0)-(D(s) \phi)^{(m-1)}=\phi^{(m-1)}(0) .
\end{aligned}
$$

Here we have used that $(\eta, \phi) \in \mathcal{M}_{m}$ and

$$
\begin{aligned}
& F_{m-1}(s)+\sum_{i=1}^{k} B_{i}(s) \phi^{(m-1)}\left(-r_{i}\right)+\int_{-\infty}^{0} B(s, \sigma) \phi^{(m-1)}(\sigma) d \sigma \\
& =\phi^{(m-1)}(0)-(D(s) \phi)^{(m-1)} .
\end{aligned}
$$


Consider the equation

$$
\begin{aligned}
v(t)= & \sum_{i=1}^{k} B_{i}(t) v\left(t-r_{i}\right)+\int_{-\infty}^{0} B(t, \sigma) v(t+\sigma) d \sigma \\
& +\frac{d^{m-1}}{d t^{m-1}} L(t) x_{t}+f^{(m)}(t)+F_{m}(t) \quad \text { a.e. on }[s, T] \\
v(t)= & \phi^{(m)}(t-s) \quad \text { a.e. on }(-\infty, 0) .
\end{aligned}
$$

Continuity of $x^{(m-1)}$ and $\left(\mathrm{H} 3_{m}\right)$ imply that

$$
\frac{d^{m-1}}{d t^{m-1}} L(t) x_{t}+f^{(m)}(t)+F_{m}(t) \in L^{2}\left(s, T ; \mathbf{R}^{n}\right) .
$$

According to Theorem 2.1 (with obvious modifications) there exists a unique solution $v$ of $(3.23)$ on $(-\infty, T]$ such that $v_{t} \in L_{g}^{2}\left(-\infty, 0 ; \mathbf{R}^{n}\right)$ for all $t \in[s, T]$. We shall show that $v(t)=x^{(m)}(t)$ and define

$$
\bar{y}(t)=\phi^{(m-1)}(0)+\int_{s}^{t} v(\sigma) d \sigma, \quad t \in(-\infty, T] .
$$

Then integration by parts yields

$$
\begin{aligned}
\int_{s}^{t} B_{i}(\tau) v\left(\tau-r_{i}\right) d \tau= & B_{t}(t) \bar{y}\left(t-r_{i}\right)-\int_{s}^{t} \dot{B}_{i}(\tau) \bar{y}\left(\tau-r_{i}\right) d \tau \\
& -B_{i}(s) \phi^{(m-1)}\left(-r_{i}\right), \quad i=1, \ldots, k
\end{aligned}
$$

and

$$
\begin{aligned}
\int_{s}^{t} \int_{-\infty}^{0} B(\tau, \sigma) v(\tau+\sigma) d \sigma d \tau= & \int_{-\infty}^{0} B(t, \sigma) \bar{y}(t+\sigma) d \sigma \\
& -\int_{s}^{t} \int_{-\infty}^{0} B_{t}(\tau, \sigma) \bar{y}(\tau+\sigma) d \sigma d \tau \\
& -\int_{-\infty}^{0} B(s, \sigma) \phi^{(m-1)}(\sigma) d \sigma
\end{aligned}
$$

Integrating (3.24) from $s$ to $t$ and using (3.24), (3.25), and (3.21) we obtain for $w(t)=\bar{y}(t)-x^{(m-1)}(t)$ :

$$
\begin{gathered}
w(t)=\sum_{i=1}^{k} B_{i}(t) w\left(t-r_{i}\right)+\int_{-\infty}^{0} B(t, \sigma) w(t+\sigma) d \sigma-\sum_{i=1}^{k} \int_{s}^{t} \dot{B}_{i}(\tau) w\left(\tau-r_{i}\right) d \tau \\
-\int_{s}^{t} \int_{-\infty}^{0} B_{t}(\tau, \sigma) w(\tau+\sigma) d \sigma d t+M \quad \text { a.e. on }[s, T], \\
w(t)=0 \text { for } t<s,
\end{gathered}
$$

where

$$
\begin{aligned}
M= & \phi^{(m-1)}(0)-f^{(m-1)}(s)-L(s) \phi^{(m-2)} \\
& -\left[F_{m-1}(s)+\sum_{i=1}^{k} B_{i}(s) \phi^{(m-1)}\left(-r_{i}\right)+\int_{-\infty}^{0} B(s, \sigma) \phi^{(m-1)}(\sigma) d \sigma\right]
\end{aligned}
$$


From (3.22) and $(\eta, \phi) \in \mathcal{M}_{m}$ we see that $M=0$. Therefore $w(t)=0$ on $[s, T]$ also and consequently $v(t)=x^{(m)}(t)$ a.e. on $(-\infty, T]$. It is easy to see that $x_{t} \in H_{g}^{m}\left(-\infty, 0 ; \mathbf{R}^{n}\right)$ for all $t \in[s, T]$. The case $m=1$ is handled in an analogous manner and the proof is finished.

3.2. Proof OF THEOREM 2.4. In this case $Y=H^{m}\left(s, T ; \mathbf{R}^{n}\right)$. We first derive the following a priori estimate of the solution $x$ which is guaranteed to exist and to be sufficiently smooth by Proposition 2.3(b).

LEMMA 3.2. Let $(\mathrm{H} 1),(\mathrm{H} 2)$, and $\left(\mathrm{H} 3_{m}\right)$ be satisfied and assume $(\eta, \phi) \in \mathcal{M}_{m}$, $m \geq 1$. Then the solution $x=x(\cdot ; \eta, \phi, f, q)$ of (1.1) satisfies

$$
\begin{aligned}
|x|_{m, \mu} & =|U(\eta, \phi, f, q) x|_{m, \mu} \\
& \leq c_{1}|q|_{Q_{m}}|\phi|_{H_{g}^{m}}+c_{2}|f|_{H^{m}}+\rho(\mu)|q|_{Q_{m}}|x|_{m, \mu}
\end{aligned}
$$

ProOF. We prove the case $m=1$ in some detail. The general case $m \geq 2$ can be verified by induction. Inequality (3.26) will follow from a series of estimates which are quite similar to those in the proof of Lemma 3.1. It is easy to see that

$$
\left|\sum_{i=1}^{k} B_{i}(\cdot) \dot{x}\left(\cdot-r_{i}\right)\right|_{\mu} \leq\left(c|\phi|_{H_{g}^{1}}+\rho(\mu)|\dot{x}|_{\mu}\right) \sum_{i=1}^{k}\left|B_{i}\right|_{H^{1}}
$$

Further, the estimate of $\left|\int_{-\infty}^{0} B(\cdot, \sigma) \dot{x}(\cdot+\sigma) d \sigma\right|_{\mu}$ is similar to that in (3.9). We have, using the continuous embedding $H^{1}\left(L_{1 / g}^{2}\right) \rightarrow C\left(L_{1 / g}^{2}\right)$

$$
\begin{array}{rl}
\mid \int_{-\infty}^{0} & \left.B(\cdot, \sigma) \dot{x}(\cdot+\sigma) d \sigma\right|_{\mu} ^{2} \\
\quad \leq & 2 \int_{s}^{T}\left(\int_{-\infty}^{0}|B(t, \sigma+s-t) \| \dot{\phi}(\sigma)| d \sigma\right)^{2} d t \\
& +2 \int_{s}^{T} e^{2 \mu(t-s)}\left(\int_{s}^{t}|B(t, \sigma-t)||\dot{x}(\sigma)| d \sigma\right)^{2} d t \\
\quad \leq & c_{1}|B|_{L^{2}\left(L_{1 / g}^{2}\right)}^{2}|\dot{\phi}|_{L_{g}^{2}}^{2}+c_{2}|B|_{H^{1}\left(L_{1 / g}^{2}\right)}^{2} \int_{s}^{T} \int_{s}^{t} e^{2 \mu(t-\sigma)} e^{2 \mu(\sigma-s)}|\dot{x}(\sigma)|^{2} d \sigma d \tau \\
\leq & c_{1}|B|_{L^{2}\left(L_{1 / g}^{2}\right)}^{2}|\dot{\phi}|_{L_{g}^{2}}^{2}+\frac{c}{2|\mu|}|B|_{H^{1}\left(L_{1 / g}^{2}\right)}^{2}|\dot{x}|_{\mu}^{2},
\end{array}
$$

so that

$$
\left|\int_{-\infty}^{0} B(\cdot, \sigma) \dot{x}(\cdot+\sigma) d \sigma\right|_{\mu} \leq\left(c|\phi|_{H_{g}^{1}}+\rho(\mu)|x|_{1, \mu}\right)|B|_{H^{1}\left(L_{1 / g}^{2}\right)}
$$


In the following estimate we use continuity of $x$ at $s$ :

$$
\begin{aligned}
& \left|\dot{B}_{i}(\cdot) x\left(\cdot-r_{i}\right)\right|_{\mu}^{2} \leq \int_{-r_{i}}^{0}\left|\dot{B}_{i}\left(t+s+r_{i}\right)\right|^{2}|\phi(t)|^{2} d t \\
& \quad+e^{2 \mu r_{1}} \int_{s}^{T-r_{i}} e^{2 \mu(t-s)}\left|\dot{B}_{i}\left(t+r_{i}\right)\right|^{2}|x(t)|^{2} d t \\
& \leq c\left|\dot{B}_{i}\right|_{L^{2}}^{2}|\phi|_{H_{g}^{1}}^{2}+e^{2 \mu r_{1}} \int_{s}^{T-r_{i}} e^{2 \mu(t-s)}\left|\dot{B}_{i}\left(t+r_{i}\right)\right|^{2}\left(|\phi(0)|+\int_{s}^{t}|\dot{x}(\sigma)| d \sigma\right)^{2} d t \\
& \leq c\left|\dot{B}_{i}\right|_{L^{2}}^{2}|\phi|_{H_{g}^{1}}^{2}+2 e^{2 \mu r_{1}} \int_{s}^{T-r_{i}} e^{2 \mu(t-s)}\left|\dot{B}_{i}\left(t+r_{i}\right)\right|^{2}\left(\int_{s}^{t}|\dot{x}(\sigma)| d \sigma\right)^{2} d t \\
& \leq c\left|\dot{B}_{i}\right|_{L^{2}}^{2}|\phi|_{H_{g}^{1}}^{2}+2 e^{2 \mu r_{1}}(T-s) \int_{s}^{T-r_{i}}\left|\dot{B}_{i}\left(t+r_{i}\right)\right|^{2} \int_{s}^{t} e^{\mu(t-s)}|\dot{x}(\sigma)|^{2} d \sigma d t \\
& \leq c\left|\dot{B}_{i}\right|_{L^{2}}^{2}|\phi|_{H_{g}^{1}}^{2}+2 e^{2 \mu r_{1}}(T-s) \int_{s}^{T-r_{i}}\left|\dot{B}_{i}\left(t+r_{i}\right)\right|^{2} \int_{s}^{t} e^{2 \mu(\sigma-s)}|\dot{x}(\sigma)|^{2} d \sigma d t \\
& \leq c\left|\dot{B}_{i}\right|_{L^{2}}^{2}|\phi|_{H_{g}^{1}}^{2}+2 e^{2 \mu r_{1}}(T-s)\left|\dot{B}_{i}\right|_{L^{2}}^{2}|x|_{1, \mu}^{2} .
\end{aligned}
$$

This implies

$$
\left|\sum_{i=1}^{k} \dot{B}_{i}(\cdot) x\left(\cdot-r_{i}\right)\right|_{\mu} \leq\left(c|\phi|_{H_{g}^{1}}+\rho(\mu)|x|_{1, \mu}\right) \sum_{i=1}^{k}\left|B_{i}\right|_{H^{1}} .
$$

The following estimate is similar to the one leading to (3.9):

$$
\left|\int_{-\infty}^{0} B_{t}(\cdot, \sigma) x(\cdot+\sigma) d \sigma\right|_{\mu}^{2} \leq \mathrm{I}+\mathrm{II}
$$

where

and

$$
\mathrm{I} \leq c\left|B_{t}\right|_{L^{2}\left(L_{1 / g}^{2}\right)}^{2}|\phi|_{L_{g}^{2}}^{2}
$$

$$
\begin{aligned}
\mathrm{II} \leq & c \int_{s}^{T}\left|B_{t}(t, \cdot)\right|_{L_{1 / g}^{2}}^{2} \int_{s}^{t} e^{2 \mu(t-s)}\left(|\phi(0)|+\int_{s}^{\sigma}|\dot{x}(\tau)| d \tau\right)^{2} d \sigma d t \\
\leq & c_{1}\left|B_{t}\right|_{L^{2}\left(L_{1 / g}^{2}\right)}^{2}|\phi|_{H_{g}^{1}} \\
& +c_{2} \int_{s}^{T}\left|B_{t}(t, \cdot)\right|_{L_{1 / g}^{2}}^{2} \int_{s}^{t}\left(\int_{s}^{\sigma} e^{\mu(t-\tau)} e^{\mu(\tau-s)}|\dot{x}(\tau)| d \tau\right)^{2} d \sigma d t \\
\leq & c_{1}\left|B_{t}\right|_{L^{2}\left(L_{1 / g}^{2}\right)}^{2}|\phi|_{H_{g}^{1}}^{2} \\
& +c_{2} \int_{s}^{T}\left|B_{t}(t, \cdot)\right|_{L_{1 / g}^{2}}^{2} \int_{s}^{t}\left(\int_{s}^{\sigma} e^{2 \mu(\tau-s)}|\dot{x}(\tau)|^{2} d \tau\right)\left(\int_{s}^{\sigma} e^{2 \mu(t-\tau)} d \tau\right) d \sigma d t \\
\leq & c\left|B_{t}\right|_{L^{2}\left(L_{1 / g}^{2}\right)}^{2}|\phi|_{H_{g}^{1}}^{2}+\rho(\mu)\left|B_{t}\right|_{L^{2}\left(L_{1 / g}^{2}\right)}^{2}|\dot{x}|_{\mu}^{2}
\end{aligned}
$$

and therefore

$$
\left|\int_{-\infty}^{0} B_{t}(\cdot, \sigma) x(\cdot+\sigma) d \sigma\right|_{\mu} \leq\left(c|\phi|_{H_{g}^{1}}+\rho(\mu)|x|_{1, \mu}\right)|B|_{H^{1}\left(L_{1 / g}^{2}\right)} .
$$


The next two estimates are completely analogous to those in (3.29) and (3.30):

$$
\begin{aligned}
& \left|\sum_{i=1}^{k} A_{i}(\cdot) x\left(\cdot-r_{i}\right)\right|_{\mu} \leq\left(c|\phi|_{H_{g}^{1}}+\rho(\mu)|x|_{1, \mu}\right) \sum_{i=1}^{k}\left|A_{i}\right|_{L^{2}}, \\
& \left|\int_{-\infty}^{0} A(\cdot, \sigma) x(\cdot+\sigma) d \sigma\right|_{\mu} \leq\left(c|\phi|_{H_{g}^{1}}+\rho(\mu)|x|_{1, \mu}\right)|A|_{L^{2}\left(L_{1 / g}^{2}\right)} .
\end{aligned}
$$

Finally we estimate the term $A_{0}(t) x(t)$ using the Minkowski integral inequality, Cauchy's inequality and Fubini's theorem:

$$
\begin{aligned}
\left|A_{0}(\cdot) x(\cdot)\right|_{\mu}^{2} & \leq 2 \int_{s}^{T} e^{2 \mu(t-s)}\left|A_{0}(t)\right|^{2}|\phi(0)|^{2} d t \\
& +2 \int_{s}^{T} e^{2 \mu(t-s)}\left|A_{0}(t)\right|^{2}\left(\int_{s}^{t}|\dot{x}(\tau)| d \tau\right)^{2} d t \\
\leq & c\left|A_{0}\right|_{L^{2}}^{2}|\phi|_{H_{g}^{1}}^{2}+2\left(\int_{s}^{T}\left(\int_{\tau}^{T} e^{2 \mu(t-s)}\left|A_{0}(t)\right|^{2}|\dot{x}(\tau)|^{2} d t\right)^{1 / 2} d \tau\right)^{2} \\
\leq & \leq\left|A_{0}\right|_{L^{2}}^{2}|\phi|_{H_{g}^{1}}^{2}+2\left(\int_{s}^{T} e^{\mu(\tau-s)}|\dot{x}(\tau)|\left(\int_{\tau}^{T} e^{2 \mu(t-\tau)}\left|A_{0}(t)\right|^{2} d t\right)^{1 / 2} d \tau\right)^{2} \\
\leq & \leq\left|A_{0}\right|_{L^{2}}^{2}|\phi|_{H_{g}^{1}}^{2}+2|\dot{x}|_{\mu}^{2} \int_{s}^{T} \int_{\tau}^{T} e^{2 \mu(t-\tau)}\left|A_{0}(t)\right|^{2} d t d \tau \\
\leq & \leq\left|A_{0}\right|_{L^{2}}^{2}|\phi|_{H_{g}^{1}}^{2}+\frac{1}{|\mu|}|x|_{1, \mu}^{2} \int_{s}^{T}\left|A_{0}(t)\right|^{2} d t
\end{aligned}
$$

and thus

$$
\left|A_{0}(\cdot) x(\cdot)\right|_{\mu} \leq\left(c|\phi|_{H_{g}^{1}}+\rho(\mu)|x|_{1, \mu}\right)\left|A_{0}\right|_{L^{2}} .
$$

The estimates (3.27)-(3.33) together with Lemma 3.1(b) imply

$$
|x|_{1, \mu} \leq\left(c_{1}|\phi|_{H_{g}^{1}}+\rho(\mu)|x|_{1, \mu}\right)|q|_{Q_{1}}+c_{2}|f|_{H^{1}}
$$


which proves the claim for $m=1$. For $m \geq 2$ we have

$$
\begin{aligned}
& x^{(m)}(t)=\sum_{i=1}^{k} B_{i}(t) x^{(m)}\left(t-r_{i}\right)+\int_{-\infty}^{0} B(t, \sigma) x^{(m)}(t+\sigma) d \sigma \\
& +\sum_{i=1}^{k} \sum_{j=0}^{m-1}\left(\begin{array}{c}
m \\
j
\end{array}\right) B_{i}^{(m-j)}(t) x^{(j)}\left(t-r_{i}\right) \\
& +\sum_{j=0}^{m-1}\left(\begin{array}{c}
m \\
j
\end{array}\right) \int_{-\infty}^{0}\left(\frac{\partial^{m-j}}{\partial t^{m-j}} B(t, \sigma)\right) x^{(j)}(t+\sigma) d \sigma \\
& +\sum_{i=0}^{k} \sum_{j=0}^{m-1}\left(\begin{array}{c}
m-1 \\
j
\end{array}\right) A_{i}^{(m-1-j)}(t) x^{(j)}\left(t-r_{i}\right) \\
& +\sum_{j=0}^{m-1}\left(\begin{array}{c}
m-1 \\
j
\end{array}\right) \int_{-\infty}^{0}\left(\frac{\partial^{m-j-1}}{\partial t^{m-j-1}} A(t, \sigma)\right) x^{(j)}(t+\sigma) d \sigma+f^{(m)}(t) .
\end{aligned}
$$

For the first two terms we use analogs to inequalities (3.27), (3.29) whereas for the other terms we use inequalities of type (3.29), (3.30), and (3.33).

PROOF OF THEOREM 2.4. (a) As in the proof of Theorem 2.1(a), we find by Proposition 2.3 that $\left(y(t)-f(t), x_{t}\right) \in \mathbf{R}^{n} \times H_{g}^{m}$ for all $t \in[s, T]$ and that $\Phi_{q}(\eta, \phi, f) \in C\left(s, T ; \mathbf{R}^{n} \times H_{g}^{m}\right)$ if $(\eta, \phi, f, q) \in \mathcal{M}_{m} \times H^{m} \times Q_{m}$.

(b) Choose $\gamma$ such that $|q|_{Q_{m}} \leq \gamma$ for $q \in S$ and then $\mu$ such that $\tilde{\alpha}=\rho(\mu) \gamma<1$. Then by Lemma 3.2 we find for all $(\eta, \phi) \in \mathcal{M}_{m}\left(f_{1}\right)$ and $(\theta, \psi) \in \mathcal{M}_{m}\left(f_{2}\right)$

$$
\left|x\left(\cdot ; \eta, \phi, f_{1}, q\right)-x\left(\cdot ; \theta, \psi, f_{2}, q\right)\right|_{m, \mu} \leq \frac{1}{1-\tilde{\alpha}}\left(c_{1} \gamma|\phi-\psi|_{H^{m}}+c_{2}\left|f_{1}-f_{2}\right|_{H^{m}}\right)
$$

for $q \in S$. Moreover,

$$
\begin{aligned}
\mid x_{t}\left(\eta, \phi, f_{1}, q\right)- & \left.x_{t}\left(\theta, \psi, f_{2}, q\right)\right|_{H_{g}^{m}} \leq(\underset{[s-T, 0]}{\operatorname{supess} G})^{1 / 2}|\phi-\psi|_{H_{g}^{m}} \\
& +\left(\operatorname{supess}_{[s-T, 0]}\right)^{1 / 2} e^{\mu(s-T)}\left|x\left(\cdot ; \eta, \phi, f_{1}, q\right)-x\left(\cdot ; \theta, \psi, f_{2}, q\right)\right|_{m, \mu} .
\end{aligned}
$$

These inequalities together with (3.16) imply (b).

(c) Let $x_{i}(t)=x\left(t ; \eta, \phi, f, q_{i}\right), i=1,2$, with $q_{i} \in S \subset Q_{m}$. Then the estimates of Lemma 3.2 imply

$$
\begin{aligned}
\left|x_{1}-x_{2}\right|_{m, \mu} & \leq\left|U\left(0,0,0, q_{1}\right)\left(x_{1}-x_{2}\right)\right|_{m, \mu}+\left|U\left(0, \phi, 0, q_{1}-q_{2}\right) x_{2}\right|_{m, \mu} \\
& \leq \rho(\mu) \gamma\left|x_{1}-x_{2}\right|_{m, \mu}+\left(c|\phi|_{H_{g}^{m}}+\rho(\mu)\left|x_{2}\right|_{m, \mu}\right)\left|q_{1}-q_{2}\right|_{Q_{m}} .
\end{aligned}
$$

As above we choose $\mu$ such that $\tilde{\alpha}=\gamma \rho(\mu)<1$. Then

$$
\left|x_{1}-x_{2}\right|_{m, \mu} \leq \frac{c}{1-\tilde{\alpha}}\left(|\phi|_{H_{g}^{m}}+\left|x_{2}\right|_{m, \mu}\right)\left|q_{1}-q_{2}\right|_{Q_{m}} .
$$

Therefore, similar to (3.17) we have

$$
\sup _{[s, T]}\left|\left(x_{1}\right)_{t}-\left(x_{2}\right)_{t}\right|_{H_{g}^{m}} \leq c\left(|\phi|_{H_{g}^{m}}+\left|x_{2}\right|_{m, \mu}\right)\left|q_{1}-q_{2}\right|_{Q_{m}}, \quad q_{1}, q_{2} \in S .
$$


Since by Lemma 3.2

$$
\left|x_{2}\right|_{m, \mu} \leq \frac{c}{1-\tilde{\alpha}}\left(|\phi|_{H_{g}^{m}}+|f|_{H^{m}}\right)
$$

we obtain

$$
\sup _{[s, T]}\left|\left(x_{1}\right)_{t}-\left(x_{2}\right)_{t}\right|_{H_{g}^{m}} \leq c\left(|f|_{H^{m}}+|\phi|_{H_{g}^{m}}\right)\left|q_{1}-q_{2}\right|_{Q_{m}}
$$

for all $q_{1}, q_{2} \in S$. This estimate together with (3.18)-(3.20) ends the proof.

3.3. Proof OF TheOREM 2.5. In this case $Y=H^{m+\alpha}\left(s, T ; \mathbf{R}^{n}\right), \alpha \in$ $(0,1), \alpha \neq \frac{1}{2}$. We first state an analogon to Lemma 3.1 and 3.2. From Proposition 2.3 we know that a solution $x(t)=x(t ; \eta, \phi, f, q)$ exists.

LEMMA 3.3. Let (H1), (H2), and $\left(\mathrm{H} 3_{m+\alpha}\right)$ be satisfied and assume $(\eta, \phi) \in$ $\mathcal{M}_{m+\alpha}, \alpha \in(0,1), \alpha \neq \frac{1}{2}$. Then the solution $x=x(\cdot ; \eta, \phi, f, q)$ satisfies

$$
\begin{aligned}
|x|_{m+\alpha, \mu}= & |U(\eta, \phi, f, q) x|_{m+\alpha, \mu} \\
\leq & c_{1}\left(1+|\mu| e^{2|\mu|(T-s)}\right)^{1 / 2}|q|_{Q_{m+\alpha}}|\phi|_{H_{g}^{m+\alpha}}+c_{2}|f|_{H^{m+\alpha}} \\
& +\rho(\mu)|q|_{Q_{m+\alpha}}|x|_{m+\alpha, \mu} .
\end{aligned}
$$

The proof of this lemma will be given below.

PROOF OF THEOREM 2.5. (a) Let $q \in Q_{m+\alpha}$. Note that the set $\mathcal{M}_{m+\alpha} \times$ $H^{m+\alpha}\left(\mathbf{R}^{n}\right)$ is a closed affine subspace of $\mathbf{R}^{n} \times H_{g}^{m+\alpha}\left(\mathbf{R}^{n}\right) \times H^{m+\alpha}\left(\mathbf{R}^{n}\right)$. We show that

$$
\Phi_{q}(\eta, \phi, f) \in C\left(s, T ; \mathbf{R}^{n} \times H_{g}^{m+\alpha}\right)
$$

for

$$
(\eta, \phi, f) \in \mathcal{M}_{m+\alpha} \times H^{m+\alpha}\left(\mathbf{R}^{n}\right) .
$$

In case $\alpha \in\left(0, \frac{1}{2}\right)$ we have

$$
\begin{aligned}
\left|x_{t}\right|_{H_{g}^{m+\alpha}}=\left|x_{t}\right|_{H_{g}^{m}} & +\left(\int_{0}^{1} \tau^{-1-2 \alpha}\left|x_{t}^{(m)}(\cdot)-x_{t}^{(m)}(\cdot+\tau)\right|_{L_{g}^{2}\left(-\infty,-\tau ; \mathbf{R}^{n}\right)}^{2} d \tau\right)^{1 / 2} \\
\leq\left|x_{t}\right|_{H_{g}^{m}}+ & \left(\int_{0}^{1} \tau^{-1-2 \alpha} \int_{-\infty}^{-\tau}\left|\phi^{(m)}(\sigma)-\phi^{(m)}(\sigma+\tau)\right|^{2} g(\sigma-\tau+s) d \sigma\right. \\
& +2 \int_{0}^{1} \tau^{-1-2 \alpha} \int_{-\tau}^{\min (0, t-s-\tau)}\left|\phi^{(m)}(\sigma)\right|^{2} g(\sigma+s-t) d \sigma d \tau \\
& +2 \int_{0}^{1} \tau^{-1-2 \alpha} \int_{0}^{\min (\tau, t-s)}\left|x^{(m)}(\sigma+s)\right|^{2} g(\sigma+s-t-\tau) d \sigma d \tau \\
& +\int_{0}^{1} \tau^{-1-1 \alpha} \int_{0}^{\max (t-s-\tau, 0)}\left|x^{(m)}(s+\sigma)-x^{(m)}(s+\sigma+h)\right|^{2} \\
& g(\sigma+s-t) d \sigma d \tau)^{1 / 2} .
\end{aligned}
$$

Let

$$
\gamma=e^{-\mu}\left[\max \left(\sup _{[s-T, 0]} G, \operatorname{supess}_{[s-T-1,0]} g\right)\right]^{1 / 2}
$$


Then Lemma 1.12(a) implies

$$
\begin{aligned}
&\left|x_{t}\right|_{H_{g}^{m+\alpha} \leq\left|x_{t}\right|_{H_{g}^{m}}+\gamma}\left(\int_{0}^{1} \tau^{-1-2 \alpha}\left|\phi^{(m)}(\cdot)-\phi^{(m)}(\cdot+\tau)\right|_{L_{g}^{2}\left(-\infty,-\tau ; \mathbf{R}^{n}\right)}^{2} d \tau\right. \\
&+2 \int_{0}^{1} \tau^{-1-2 \alpha} \int_{-\tau}^{0}\left|\phi^{(m)}(\sigma)\right|^{2} d \sigma d \tau \\
&+2 \int_{0}^{1} \tau^{-1-2 \alpha} \int_{0}^{\tau}\left|x^{(m)}(\sigma+s)\right|^{2} e^{2 \mu \sigma} d \sigma d \tau \\
&\left.+\int_{0}^{1} \tau^{-1-2 \alpha} \int_{0}^{T-s-\tau}\left|x^{(m)}(s+\sigma)-x^{(m)}(s+\sigma+\tau)\right|^{2} e^{2 \mu \sigma} d \sigma d \tau\right)^{1 / 2} \\
& \leq c\left(|\phi|_{H_{g}^{m+\alpha}}+|x|_{m+\alpha, \mu}\right) .
\end{aligned}
$$

Similarly, for $\alpha \in\left(\frac{1}{2}, 1\right)$ we get by Lemma $1.12(\mathrm{~b})$

$$
\begin{aligned}
\left|x_{t}\right|_{H_{g} m+\alpha} \leq\left|x_{t}\right|_{H_{g}^{m}}+( & \int_{0}^{1} \tau^{-1-2 \alpha} \int_{-\infty}^{-\tau}\left|\phi^{(m)}(\sigma)-\phi^{(m)}(\sigma+\tau)\right|^{2} g(\sigma-s+t) d \sigma d \tau \\
& +2 \int_{0}^{1} \tau^{-1-2 \alpha} \int_{s-t-\tau}^{\min (s-t,-\tau)}\left|\phi^{(m)}(t+\sigma-s)-\phi^{(m)}(0)\right|^{2} g(\sigma) d \sigma d \tau \\
& +2 \int_{0}^{1} \tau^{-1-2 \alpha} \int_{s-t-\tau}^{\min (s-t,-h)}\left|x^{(m)}(s)-x^{(m)}(t+\sigma+\tau)\right|^{2} \cdot g(\sigma) d \sigma d \tau \\
& +\int_{0}^{1} \tau^{-1-2 \alpha} \int_{0}^{\max (t-s-\tau, 0)}\left|x^{(m)}(s+\sigma)-x^{(m)}(s+\sigma+\tau)\right|^{2} \\
\leq\left|x_{t}\right|_{H_{g}^{m}+\gamma}\left(|\phi|_{H_{g}^{m+\alpha}}^{2}+2 \int_{0}^{1} \tau^{-1-2 \alpha} \int_{-\tau}^{0}\left|\phi^{(m)}(\sigma)-\phi^{(m)}(0)\right|^{2} d \sigma d \tau\right. & \\
& +2 \int_{0}^{1} \tau^{-1-2 \alpha} \int_{s}^{s+\tau}\left|x^{(m)}(s)-x^{(m)}(\sigma)\right|^{2} e^{2 \mu(\sigma-s)} d \sigma d \tau \\
& \left.+\int_{0}^{1} \tau^{-1-2 \alpha} \int_{s}^{T-\tau}\left|x^{(m)}(\sigma)-x^{(m)}(\sigma+\tau)\right|^{2} e^{2 \mu(\sigma-s)} d \sigma d \tau\right)^{1 / 2} \\
\leq c\left(|\phi|_{H_{g}^{m+\alpha}+}+|x|_{m+\alpha, \mu}\right) . &
\end{aligned}
$$

Since Lemma 3.3 implies that $\mu$ can be chosen such that

$$
|x|_{m+\alpha, \mu}<c\left(\left(1+|\mu| e^{2|\mu|(T-s)}\right)^{1 / 2}|\phi|_{H_{g}^{m+\alpha}}|q|_{Q_{m+\alpha}}+|f|_{H^{m+\alpha}}\right),
$$

we obtain $\left(y(t), x_{t}\right) \in \mathbf{R}^{n} \times H_{g}^{m+\alpha}\left(\mathbf{R}^{n}\right)$ for all $t \in[s, T]$.

In order to finish the proof of part (a) of Theorem 2.5 we have to prove continuity of $t \rightarrow x_{t}$. Let $s \leq t_{1} \leq t_{2} \leq T$. Then

$$
\left|x_{t_{1}}-x_{t_{2}}\right|_{H_{g}^{m+\alpha}}^{2}=\left|x_{t_{1}}-x_{t_{2}}\right|_{H_{g}^{m}}^{2}+J\left(t_{1}, t_{2}\right) \text {, }
$$

where

$J\left(t_{1}, t_{2}\right)=\int_{0}^{1} t^{-1-2 \alpha} \int_{-\infty}^{-t}\left|x_{t_{1}}^{(m)}(\tau)-x_{t_{2}}^{(m)}(\tau)-x_{t_{1}}^{(m)}(t+\tau)+x_{t_{2}}^{(m)}(t+\tau)\right|^{2} g(\tau) d \tau d t$ 
It is clear that $\left|x_{t_{1}}-x_{t_{2}}\right|_{H_{g}^{m}} \rightarrow 0$ as $t_{2}-t_{1} \rightarrow 0$. Let $\xi \in[s, T]$ and $M>T-s$. Recall that $M>1$, since $T>s+r+1$. Then

$$
\begin{aligned}
\int_{0}^{1} t^{-1-2 \alpha} \int_{-\infty}^{-M}\left|x_{\xi}^{(m)}(\tau)-x_{\xi}^{(m)}(t+\tau)\right|^{2} g(\tau) d \tau d t \\
\quad=\int_{0}^{1} t^{-1-2 \alpha} \int_{-\infty}^{-M+\xi-s}\left|\phi^{(m)}(\sigma)-\phi^{(m)}(\sigma+t)\right|^{2} g(\sigma+s-\xi) d \sigma d t \\
\quad \leq \operatorname{supess}_{[s-T, 0]} \int_{0}^{1} t^{-1-2 \alpha} \int_{-\infty}^{-M+T-s}\left|\phi^{(m)}(\sigma)-\phi^{(m)}(\sigma+t)\right|^{2} g(\sigma) d \sigma d t \\
\quad<\varepsilon / 4 \text { for all } \xi \in[s, T]
\end{aligned}
$$

provided we choose $M$ sufficiently large. Using this we get

$$
\begin{aligned}
\int_{0}^{1} t^{-1-2 \alpha} & \int_{-\infty}^{-M}\left|x_{t_{1}}^{(m)}(\tau)-x_{t_{2}}^{m}(\tau)-x_{t_{1}}^{(m)}(t+\tau)+x_{t_{2}}^{(m)}(t+\tau)\right|^{2} g(\tau) d \tau d t \\
\leq & 2 \int_{0}^{1} t^{-1-2 \alpha} \int_{-\infty}^{-M}\left|x_{t_{1}}^{(m)}(\tau)-x_{t_{1}}^{(m)}(t+\tau)\right|^{2} g(\tau) d \tau d t \\
& \quad+2 \int_{0}^{1} t^{-1-2 \alpha} \int_{-\infty}^{-M}\left|x_{t_{2}}^{(m)}(\tau)-x_{t_{2}}^{(m)}(t+\tau)\right|^{2} g(\tau) d \tau d t \\
& <\varepsilon
\end{aligned}
$$

for all $t_{1}, t_{2} \in[s, T]$. Since we have

$$
\begin{aligned}
& \int_{0}^{1} t^{-1-2 \alpha} \int_{-M}^{-t}\left|x_{t_{1}}^{(m)}(\tau)-x_{t_{2}}^{(m)}(\tau)-x_{t_{1}}^{(m)}(t+\tau)+x_{t_{2}}^{(m)}(t+\tau)\right|^{2} g(\tau) d \tau d t \\
& \leq \sup _{[-M, 0]} g \int_{0}^{1} t^{-1-2 \alpha} \int_{-M}^{-t} \mid x_{t_{1}}^{(m)}(\tau)-x_{t_{2}}^{(m)}(\tau) \\
& -x_{t_{1}}^{(m)}(t+\tau)+\left.x_{t_{2}}^{(m)}(t+\tau)\right|^{2} d \tau d t,
\end{aligned}
$$

it remains to prove that

$$
K\left(t_{1}, t_{2}\right):=\int_{0}^{1} t^{-1-2 \alpha} \int_{-M}^{-t}\left|x_{t_{1}}^{(m)}(\tau)-x_{t_{2}}^{(m)}(\tau)-x_{t_{1}}^{(m)}(t+\tau)+x_{t_{2}}^{(m)}(t+\tau)\right|^{2} d \tau d t \rightarrow 0
$$

as $t_{2}-t_{1} \rightarrow 0$. In the last integral only values of $x(t)$ for $t \in[s-M, T]=: I_{0}$ appear. Since $x_{T} \in H_{g}^{m+\alpha}$, it is clear that $x \mid I_{0} \in H^{m+\alpha}\left(I_{0} ; \mathbf{R}^{n}\right)$ or $x^{(m)} \mid I_{0} \in H^{\alpha}\left(I_{0} ; \mathbf{R}^{n}\right)$ by the properties of $g$. Let $p_{0}$ be the extension operator corresponding to $I_{0}$ (defined with $m=1$ ). Then

$$
u=p_{0}\left(x^{(m)} \mid I_{0}\right) \in H^{\alpha}\left(\mathbf{R} ; \mathbf{R}^{n}\right) .
$$

Since the shift is strongly continuous (see Proposition 1.1$)$ on $H^{\alpha}\left(\mathbf{R} ; \mathbf{R}^{n}\right)$ we see that for any $\varepsilon>0$ there exists a $\delta>0$ such that

$$
\left|u\left(t_{1}+\cdot\right)-u\left(t_{2}+\cdot\right)\right|_{H^{\alpha}\left(\mathbf{R} ; \mathbf{R}^{n}\right)}<\varepsilon
$$

provided $t_{2}-t_{1}<\delta$. Let $r_{I}$ be the restriction operator corresponding to $I=$ $[-M, 0]$. Then

$$
\left|r_{I} u(t+\cdot)-r_{i} u\left(t_{2}+\cdot\right)\right|_{H^{\alpha}\left(I ; \mathbf{R}^{n}\right)}<c \varepsilon
$$


for $t_{2}-t_{1}<\delta$. The integral in the above norm is just

$$
\begin{aligned}
& \int_{0}^{1} t^{-1-2 \alpha} \int_{-M}^{-t}\left|u\left(t_{1}+\tau\right)-u\left(t_{2}+\tau\right)-u\left(t_{1}+t+\tau\right)+u\left(t_{2}+t+\tau\right)\right|^{2} d \tau d t \\
& =\int_{0}^{1} t^{-1-2 \alpha} \int_{-M}^{-t} \mid x^{(m)}\left(t_{1}+\tau\right)-x^{(m)}\left(t_{2}+\tau\right) \\
& =K\left(t_{1}, t_{2}\right) .
\end{aligned}
$$

With the estimates (3.35), (3.35) and Lemma 3.3 the proofs of (b) and (c) are analogous to those of Theorem 2.2 , (b) and (c).

PROOF OF LEMMA 3.3. We first consider the case $m=1$. Since the estimates for the $H^{1+\alpha}$-norm of the terms on the right side of (3.6) are rather tedious, we only give the details for three terms which are representative for the rest.

(i) Estimation of $B_{i}(t) \dot{x}\left(t-r_{i}\right), i=1, \ldots, k$. We have

$$
\left[B_{i}(\cdot) \dot{x}\left(\cdot-r_{i}\right)\right]_{\alpha, \mu}^{2}=\int_{0}^{1} t^{-1-2 \alpha} \beta(t) d t
$$

where

$$
\begin{aligned}
\beta(t)= & \int_{s}^{T-t}\left|e^{\mu(\tau-s)} B_{i}(\tau) \dot{x}\left(\tau-r_{i}\right)-e^{\mu(\tau-s+t)} B_{i}(\tau+t) \dot{x}\left(\tau-r_{i}+t\right)\right|^{2} d \tau \\
\leq & 2 \int_{s}^{T-t}\left|B_{i}(\tau)\right|^{2}\left|e^{\mu(\tau-s)} \dot{x}\left(\tau-r_{i}\right)-e^{\mu(\tau-s+t)} \dot{x}\left(\tau-r_{i}+t\right)\right|^{2} d \tau \\
& +2 \int_{s}^{T-t}\left|B_{i}(\tau)-B_{i}(\tau+h)\right|^{2}\left|e^{\mu(\tau-s+t)} \dot{x}\left(\tau-r_{i}+t\right)\right|^{2} d \tau \\
= & : \text { I }+ \text { II. }
\end{aligned}
$$

We first consider I and get using the continuous embedding $H^{1+\alpha}\left(\mathbf{R}^{n \times n}\right) \subset$ $C\left(\mathbf{R}^{n \times n}\right)$

$$
\mathrm{I} \leq 2\left|B_{i}\right|_{H^{1+\alpha}}^{2}\left(J_{1}+J_{2}+J_{3}\right)
$$

where

$$
\begin{aligned}
& J_{1}=\int_{s-r_{i}}^{s-t} e^{2 \mu\left(r_{i}+\tau-s\right)}\left|\dot{\phi}(\tau-s)-e^{\mu t} \dot{\phi}(\tau-s+t)\right|^{2} d \tau, \\
& J_{2}=\int_{s-t}^{s} e^{2 \mu\left(r_{i}+\tau-s\right)}\left|\dot{\phi}(\tau-s)-e^{\mu t} \dot{z}(\tau+t)\right|^{2} d \tau
\end{aligned}
$$

and

$$
J_{3}=\int_{s}^{T-t-r_{i}} e^{2 \mu r_{i}}\left|e^{\mu(\tau-s)} \dot{z}(\tau)-e^{\mu(\tau-s+t)} \dot{z}(\tau+t)\right|^{2} d \tau .
$$

Using $r_{i}+\tau-s \geq 0$ we get

$$
\begin{aligned}
J_{1} & \leq 2 \int_{s-r_{i}}^{s-t}|\dot{\phi}(\tau-s)-\dot{\phi}(\tau-s+t)|^{2} d \tau+2\left(1-e^{\mu t}\right)^{2} \int_{s-r_{i}}^{s-t}|\dot{\phi}(\tau+t-s)|^{2} d \tau \\
& \leq 2|\dot{\phi}(\cdot)-\dot{\phi}(\cdot+t)|_{L^{2}\left(-r,-t ; \mathbf{R}^{n}\right)}^{2}+2|\mu|^{2} t^{2}|\dot{\phi}|_{L^{2}\left(-r, 0 ; \mathbf{R}^{n}\right)}^{2} \\
& \leq 2\left(\operatorname{supess}_{1-r, 0]} \frac{1}{g}\right)\left\{|\dot{\phi}(\cdot)-\dot{\phi}(\cdot+t)|_{L_{g}^{2}}^{2}+|\mu|^{2} t^{2}|\phi|_{H_{g}^{1}}^{2}\right\} .
\end{aligned}
$$


We next consider $J_{2}$ for $\alpha \in\left(0, \frac{1}{2}\right)$. Recall that we assume $r_{1}>1$.

$$
\begin{aligned}
J_{2} & \leq 2 \int_{s-t}^{s} e^{2 \mu\left(r_{i}+\tau-s\right)}|\dot{\phi}(\tau-s)|^{2} d \tau+2 \int_{s-t}^{s} e^{2 \mu\left(r_{i}+\tau-s+t\right)}|\dot{z}(\tau+t)|^{2} d \tau \\
& \leq 2 \int_{-t}^{0}|\dot{\phi}(\tau)|^{2} d \tau+2 e^{2 \mu r_{1}} \int_{s}^{s+t} e^{2 \mu(\tau-s)}|\dot{z}(\tau)|^{2} d \tau .
\end{aligned}
$$

For $\alpha \in\left(\frac{1}{2}, 1\right)$ we obtain, using the estimates $e^{\mu \tau}-1 \leq\left(1-e^{-\mu}\right) \tau$ for $-1 \leq \tau \leq 0$ and

$$
\begin{aligned}
& \int_{s}^{s+t}\left(e^{\mu(\tau-s-t)}-1\right)^{2} d \tau \leq \frac{1}{3} t^{3} e^{-2 \mu} \\
& J_{2} \leq 2 \int_{s-t}^{s} e^{2 \mu\left(r_{i}+\tau+s\right)}|\dot{\phi}(\tau-s)-\dot{\phi}(0)|^{2} d \tau \\
&+2 \int_{s-t}^{s} e^{2 \mu\left(r_{i}+\tau-s\right)}\left|\dot{z}(s)-e^{\mu t} \dot{z}(\tau+t)\right|^{2} d \tau \\
& \leq 2 \int_{-t}^{0}|\dot{\phi}(\tau)-\dot{\phi}(0)|^{2} d \tau+4 e^{2 \mu r_{1}} \int_{s}^{s+t}\left(e^{\mu(\tau-s-t)}-1\right)^{2} d \tau|\dot{z}(s)|^{2} \\
&+4 e^{2 \mu r_{1}} \int_{s}^{s+t}\left|\dot{z}(s)-e^{\mu(\tau-s)} \dot{z}(\tau)\right|^{2} d \tau \\
& \leq 2 \int_{-t}^{0}|\dot{\phi}(\tau)-\dot{\phi}(0)|^{2} d \tau+\frac{4}{3} t^{3} e^{2 \mu\left(r_{1}-1\right)}|\dot{z}(s)|^{2} \\
&+4 e^{2 \mu r_{1}} \int_{s}^{s+t}\left|\dot{z}(s)-e^{\mu(\tau-s)} \dot{z}(\tau)\right|^{2} d \tau .
\end{aligned}
$$

For $J_{3}$ we have the obvious estimate

$$
J_{3} \leq e^{2 \mu r_{1}}\left|\dot{z}(\cdot) e^{\mu(\cdot-s)}-z(\cdot+t) e^{\mu(\cdot-s+t)}\right|_{L^{2}\left(s, T-t ; \mathbf{R}^{n}\right)}^{2} .
$$

Summarizing these estimates and using Lemma 1.12 we obtain for $\alpha \in(0,1), \alpha \neq \frac{1}{2}$,

$$
\int_{0}^{1} t^{-1-2 \alpha} I d t \leq c\left|B_{i}\right|_{H^{1+\alpha}}^{2}\left((1+|\mu|)|\phi|_{H_{g}^{1+\alpha}}^{2}+e^{2 \mu\left(r_{1}-1\right)}|z|_{1+\alpha, \mu}\right) .
$$

We still have to consider II:

$$
\begin{aligned}
\mathrm{II} & \leq 2 \int_{s}^{T-t}\left(\int_{\tau}^{\tau+t}\left|\dot{B}_{i}(\sigma)\right| d \sigma\right)^{2}\left|e^{\mu(\tau-s+t)} \dot{x}\left(\tau-r_{i}+t\right)\right|^{2} d \tau \\
& \leq 2 t \int_{s}^{T-t} \int_{\tau}^{\tau+t}\left|\dot{B}_{i}(\sigma)\right|^{2} d \sigma\left|e^{\mu(\tau-s+t)} \dot{x}\left(\tau-r_{i}+t\right)\right|^{2} d \tau .
\end{aligned}
$$

In case $\alpha \in\left(0, \frac{1}{2}\right)$ we use

$$
\int_{\tau}^{T+t}\left|\dot{B}_{i}(\sigma)\right|^{2} d \sigma \leq\left|B_{i}\right|_{H^{1}}^{2} \leq\left|B_{i}\right|_{H^{1+\alpha}}^{2}
$$


and get

$$
\begin{aligned}
\mathrm{II} & \leq 2 t\left|B_{i}\right|_{H^{1+\alpha}}^{2}\left(\int_{s-r_{i}+t}^{s} e^{2 \mu\left(r_{i}+\tau-s\right)}|\dot{\phi}(\tau-s)|^{2} d \tau\right. \\
& \left.+\int_{s}^{T-r_{i}} e^{2 \mu\left(r_{i}+\tau-s\right)}|\dot{z}(\tau)|^{2} d \tau\right) \\
& \leq 2 t\left|B_{i}\right|_{H^{1+\alpha}}^{2}\left(|\phi|_{H^{1}\left(-r, 0 ; \mathbf{R}^{n}\right)}^{2}+e^{2 \mu r_{1}}|z|_{1, \mu}^{2}\right) \\
& \leq 2 t\left|B_{i}\right|_{H^{1+\alpha}}^{2}\left(\left(\operatorname{supess}_{[-r, 0]} \frac{1}{g}\right)|\phi|_{H_{g}^{1+\alpha}}^{2}+e^{2 \mu r_{1}}|z|_{1+\alpha, \mu}^{2}\right) .
\end{aligned}
$$

In case $\alpha \in\left(\frac{1}{2}, 1\right)$ we use the continuous embedding $H^{\alpha}\left(\mathbf{R}^{n \times n}\right) \subset C\left(\mathbf{R}^{n \times n}\right)$ and get with $\int_{\tau}^{\tau+t}\left|\dot{B}_{i}(\sigma)\right|^{2} d \sigma \leq c t\left|B_{i}\right|_{H^{1+\alpha}}^{2}$ the estimate

$$
\begin{aligned}
\mathrm{II} & \leq c t^{2}\left|B_{i}\right|_{H^{1+\alpha}}^{2} \int_{s-r_{i}+t}^{T-r_{i}} e^{2 \mu\left(r_{i}+\tau-s\right)}|\dot{x}(\tau)|^{2} d \tau \\
& \leq c t^{2}\left|B_{i}\right|_{H^{1+\alpha}}^{2}\left(\left(\operatorname{supess}_{[-r, 0]} \frac{1}{g}\right)|\phi|_{H_{g}^{1+\alpha}}^{2}+e^{2 \mu r_{1}}|z|_{1+\alpha, \mu}^{2}\right) .
\end{aligned}
$$

Thus for $\alpha \in(0,1), \alpha \neq \frac{1}{2}$,

$$
\int_{0}^{1} t^{-1-2 \alpha} \mathrm{II} d t \leq c\left|B_{i}\right|_{H^{1+\alpha}}^{2}\left(|\phi|_{H_{g}^{1+\alpha}}^{2}+e^{2 \mu r_{1}}|z|_{1+\alpha, \mu}^{2}\right) .
$$

Inequalities (3.37) and (3.38) together imply

$$
\left[\sum_{i=1}^{k} B_{i}(\cdot) \dot{x}\left(\cdot-r_{i}\right)\right]_{\alpha, \mu}^{2} \leq c \sum_{i=1}^{k}\left|B_{i}\right|_{H^{1+\alpha}}^{2}\left((1+|\mu|)|\phi|_{H_{g}^{1+\alpha}}^{2}+e^{2 \mu\left(r_{1}-1\right)}|z|_{1+\alpha, \mu}^{2}\right) .
$$

(ii) Estimation of $\int_{-\infty}^{0} B(t, \sigma) \dot{x}(t+\sigma) d \sigma$. We have

$$
\begin{aligned}
& {\left[\int_{-\infty}^{0} B(t, \sigma) \dot{x}(t+\sigma) d \sigma\right]_{\alpha, \mu}^{2}} \\
& \quad=\int_{0}^{1} t^{-1-2 \alpha} \int_{s}^{T-t} \mid e^{\mu(\tau-s)} \int_{-\infty}^{0} B(\tau, \sigma) \dot{x}(\tau+\sigma) d \sigma \\
& \quad-\left.e^{\mu(\tau-s+t)} \int_{-\infty}^{0} B(\tau+t, \sigma) \dot{x}(\tau+\sigma+t) d \sigma\right|^{2} d \tau d t \\
& =: \int_{0}^{1} t^{-1-2 \alpha} \beta(t) d t .
\end{aligned}
$$

We split $\beta(t)$ as follows:

$$
\begin{aligned}
\beta(t) \leq & 2 \int_{s}^{T-t}\left|\int_{-\infty}^{0} B(\tau+t, \sigma)\left[\dot{x}(\tau+\sigma) e^{\mu(\tau-s)}-\dot{x}(\tau+\sigma+t) e^{\mu(\tau-s+t)}\right] d \sigma\right|^{2} d \tau \\
& +2 \int_{s}^{T-t}\left|e^{\mu(\tau-s)} \int_{-\infty}^{0}[B(\tau, \sigma)-B(\tau+t, \sigma)] \dot{x}(\tau+\sigma) d \sigma\right|^{2} d \tau \\
= & \text { I }+ \text { II. }
\end{aligned}
$$


For I we get

$$
\begin{aligned}
\mathrm{I} & \leq 2 \int_{s}^{T-t}\left(\int_{-\infty}^{0} \frac{|B(\tau+t, \sigma)|^{2}}{g(\sigma)} d(\sigma)\right) \\
& \cdot\left(\int_{-\infty}^{0}\left|e^{\mu(\tau-s)} \dot{x}(\tau+\sigma)-e^{\mu(\tau-s+t)} \dot{x}(\tau+\sigma+t)\right|^{2} \mid g(\sigma) d \sigma\right) d \tau \\
& \leq 2 \int_{s}^{T-t}|B(\tau+t, \cdot)|_{L_{1 / g}^{2}}^{2} \int_{-\infty}^{\tau}\left|e^{\mu(\tau-s)} \dot{x}(\sigma)-e^{\mu(\tau-s+t)} \dot{x}(\sigma+t)\right|^{2} g(\sigma-\tau) d \sigma d \tau \\
& \leq 2|B|_{L^{\infty}\left(s, T ; L_{1 / g}^{2}\right)}^{2}\left\{J_{1}+J_{2}+J_{3}\right\} \\
& \leq 2 c|B|_{H^{1}\left(s, T ; L_{1 / g}^{2}\right)}^{2}\left\{J_{1}+J_{2}+J_{3}\right\}
\end{aligned}
$$

where

$$
\begin{aligned}
& J_{1}=\int_{s}^{T-s} \int_{-\infty}^{s-t}\left|e^{\mu(\tau-s)} \dot{\phi}(\sigma-s)-e^{\mu(\tau-s+t)} \dot{\phi}(\sigma-s+t)\right|^{2} g(\sigma-\tau) d \sigma d \tau, \\
& J_{2}=\int_{s}^{T-s} \int_{s-t}^{s}\left|e^{\mu(\tau-s)} \dot{\phi}(\sigma-s)-e^{\mu(\tau-s+t)} \dot{z}(\sigma+t)\right|^{2} g(\sigma-\tau) d \sigma d \tau, \\
& J_{3}=\int_{s}^{T-t} \int_{s}^{\tau}\left|e^{\mu(\tau-s)} \dot{z}(\sigma)-e^{\mu(\tau-s+t)} \dot{z}(\sigma+t)\right|^{2} g(\sigma-\tau) d \sigma d \tau .
\end{aligned}
$$

For $J_{1}$ we have

$$
\begin{aligned}
& J_{1} \leq 2 \int_{s}^{T-t} \int_{-\infty}^{s-t} e^{2 \mu(\tau-s)}|\dot{\phi}(\sigma-s)-\dot{\phi}(\sigma-s+t)|^{2} g(\sigma-\tau) d \sigma d \tau \\
& +2 \int_{s}^{T-t} \int_{-\infty}^{s-t} e^{\mu(\tau-s)}\left(1-e^{\mu t}\right)^{2}|\dot{\phi}(\sigma+t-s)|^{2} g(\sigma-\tau) d \sigma d \tau \\
& \leq 2 \int_{s}^{T-t} \int_{-\infty}^{-t}|\dot{\phi}(\sigma)-\dot{\phi}(\sigma+t)|^{2} g(\sigma) G(s-\tau) d \sigma d \tau \\
& +2 \int_{s}^{T-t}|\mu|^{2} t^{2} \int_{-\infty}^{0}|\dot{\phi}(\sigma)|^{2} g(\sigma) G(s-t-\tau) d \sigma d \tau \\
& \leq 2(T-s)\left(\operatorname{supess}_{[s-T, 0]}\right)|\dot{\phi}(\cdot)-\dot{\phi}(\cdot+h)|_{L_{g}^{2}}^{2} \\
& +(T-s)\left(\operatorname{supess}_{[s-T, 0]}\right)|\mu| t^{2}|\phi|_{H_{g}^{1}}^{2} \text {. }
\end{aligned}
$$

For $J_{3}$ we get

$$
\begin{aligned}
& J_{3} \leq\left(\operatorname{supess}_{[s-T, 0]}\right) \int_{s}^{T-t} \int_{s}^{\tau} e^{2 \mu(\tau-\sigma)}\left|e^{\mu(\sigma-s)} \dot{z}(\sigma)-e^{\mu(\sigma-s+t)} \dot{z}(\sigma+t)\right|^{2} d \sigma d \tau \\
& =\left(\sup _{[s-T, 0]} g\right) \int_{s}^{T-t}\left|e^{\mu(\sigma-s)} \dot{z}(\sigma)-e^{\mu(\sigma-s+t)} \dot{z}(\sigma+t)\right|^{2} \int_{\sigma}^{T-t} e^{2 \mu(\tau-\sigma)} d \tau d \sigma .
\end{aligned}
$$


Observing that $\int_{\sigma}^{T-t} e^{2 \mu(\tau-\sigma)} d \tau \leq 1 /(2|\mu|)$ we have

$$
J_{3} \leq \frac{1}{2|\mu|}\left(\operatorname{supess}_{[s-T, 0]}\right) e^{\mu(\cdot-s)} \dot{z}(\cdot)-\left.e^{\mu(\cdot-s+t)} \dot{z}(\cdot+h)\right|_{L^{2}\left(s, T-t ; \mathbf{R}^{n}\right)} ^{2}
$$

For $J_{2}$ we get in case $\alpha \in\left(0, \frac{1}{2}\right)$

$$
\begin{aligned}
J_{2} & \leq \int_{s}^{T-t} \int_{s-t}^{s}\left|e^{\mu(\tau-s)} \dot{\phi}(\sigma-s)-e^{\mu(\tau-s+t)} \dot{z}(\sigma+t)\right|^{2} g(\sigma-\tau) d \sigma d \tau \\
& \leq c\left\{\int_{s}^{T-t} \int_{-t}^{0} e^{2 \mu(\tau-s)}|\dot{\phi}(\sigma)|^{2} d \sigma d \tau\right. \\
& \left.+\int_{s}^{T-t} \int_{s}^{s+t} e^{2 \mu(\tau-\sigma+t)} e^{2 \mu(\sigma-s)}|\dot{z}(\sigma)|^{2} d \sigma d \tau\right\} \\
& \leq c\left\{(T-s) \int_{-t}^{0}|\dot{\phi}(\sigma)|^{2} d \sigma+\frac{1}{2|\mu|} \int_{s}^{s+t} e^{2 \mu(\sigma-s)}|\dot{z}(\sigma)|^{2} d \sigma\right\},
\end{aligned}
$$

where $c=2$ sup $\operatorname{ess}_{[s-T, 0]} g$. In case $\alpha \in\left(\frac{1}{2}, 1\right)$ we obtain

$$
\begin{aligned}
J_{2} \leq & \int_{s}^{T-t} \int_{s-t}^{s}\left|e^{\mu(\tau-s)} \dot{\phi}(\sigma-s)-e^{\mu(\tau-s+t)} \dot{z}(\sigma+t)\right|^{2} g(\sigma-\tau) d \sigma d \tau \\
\leq & c\left\{\int_{s}^{T-t} \int_{s-t}^{s}\left|e^{\mu(\tau-s)}(\dot{\phi}(\sigma-s)-\dot{\phi}(0))\right|^{2} d \sigma d \tau\right. \\
& \left.+\int_{s}^{T-t} \int_{s-t}^{s}\left|e^{\mu(\tau-s)} \dot{\phi}(0)-e^{\mu(\tau-\sigma)} e^{\mu(\sigma-s+t)} \dot{z}(\sigma+t)\right|^{2} d \sigma d \tau\right\} \\
\leq & \left\{(T-s) \int_{-t}^{0}|\dot{\phi}(\sigma)-\dot{\phi}(0)|^{2} d \sigma+2|\dot{\phi}(0)|^{2}\right. \\
\cdot \int_{s}^{T-t} \int_{s-t}^{s} e^{2 \mu(\tau-\sigma)}\left(e^{\mu(\sigma-s)}-1\right)^{2} d \sigma d \tau & \left.\left|\dot{z}(s)-e^{\mu(\sigma-s+t)} \dot{z}(\sigma+t)\right|^{2} \int_{s}^{T-t} e^{2 \mu(\tau-\sigma)} d \tau d \sigma\right\} \\
\leq c & \left\{(T-s) \int_{-t}^{0}|\dot{\phi}(\sigma)-\dot{\phi}(0)|^{2} d \sigma+|\dot{\phi}(0)|^{2}|\mu| e^{2|\mu|(T-s)} t^{3}\right. \\
& \left.+\frac{1}{|\mu|} \int_{s}^{s+t}\left|\dot{z}(s)-e^{\mu(\sigma-s)} \dot{z}(\sigma)\right|^{2} d \sigma\right\}
\end{aligned}
$$

Summarizing these estimates we obtain with Lemma 1.12

$$
\int_{0}^{1} t^{-1-2 \alpha} \mathrm{I} d t \leq c|B|_{H^{1}\left(s, T ; L_{1 / g}^{2}\right)}\left(\left(1+|\mu| e^{2|\mu|(T-s)}\right)|\phi|_{H_{g}^{1+\alpha}}^{2}+\frac{1}{|\mu|}|z|_{1+\alpha, \mu}^{2}\right) .
$$


For II we have

$$
\begin{array}{r}
\mathrm{II} \leq 2 \int_{s}^{T-t}\left(\int_{-\infty}^{0} \frac{|B(\tau, \sigma)-B(\tau+t, \sigma)|^{2}}{g(\sigma)} d \sigma\right) \\
\cdot\left(\int_{-\infty}^{0} e^{2 \mu(\tau-s)}|\dot{x}(\tau+\sigma)|^{2} g(\sigma) d \sigma\right) d \tau .
\end{array}
$$

In case $\alpha \in\left(0, \frac{1}{2}\right)$ we get

$$
\begin{aligned}
\int_{-\infty}^{0} & \frac{|B(\tau, \sigma)-B(\tau+t, \sigma)|^{2}}{g(\sigma)} d \sigma \\
& \leq \int_{-\infty}^{0} \frac{1}{g(\sigma)}\left(\int_{\tau}^{\tau+t}\left|B_{t}(\rho, \sigma)\right| d \rho\right)^{2} d \sigma \\
& \leq t \int_{-\infty}^{0} \frac{1}{g(\sigma)} \int_{\tau}^{\tau+t}\left|B_{t}(\rho, \sigma)\right|^{2} d \rho d \sigma \\
& =t \int_{\tau}^{\tau+t} \int_{-\infty}^{0} \frac{\left|B_{t}(\rho, \sigma)\right|^{2}}{g(\sigma)} d \sigma d \rho \\
& =t \int_{\tau}^{\tau+t}\left|B_{t}(\rho, \cdot)\right|_{L_{1 / g}^{2}}^{2} d \rho \leq t|B|_{H^{1}\left(s, T ; L_{1 / g}^{2}\right)}^{2}
\end{aligned}
$$

and therefore

$$
\mathrm{II} \leq 2 t|B|_{H^{1}\left(s, T ; L_{1 / g}^{2}\right)}^{2} \int_{s}^{T-t} \int_{-\infty}^{0} e^{2 \mu(\tau-s)}|\dot{x}(\tau+\sigma)|^{2} g(\sigma) d \sigma d \tau
$$

The integral can be estimated as follows:

$$
\begin{aligned}
\int_{s}^{T-t} & \int_{-\infty}^{0} e^{2 \mu(\tau-s)}|\dot{x}(\tau+\sigma)|^{2} g(\sigma) d \sigma d \tau \\
& \leq(T-s)\left(\operatorname{supess}_{[s-T, 0]}\right)|\phi|_{H_{g}^{1}}^{2}+\left(\operatorname{supess}_{[s-T, 0]}\right) \frac{1}{2|\mu|}|z|_{1, \mu}^{2} .
\end{aligned}
$$

Thus we obtain for $\alpha \in\left(0, \frac{1}{2}\right)$

$$
\mathrm{II} \leq c t|B|_{H^{1}\left(s, T ; L_{1 / g}^{2}\right)}\left(|\phi|_{H_{g}^{1}}^{2}+\frac{1}{2|\mu|}|z|_{1, \mu}^{2}\right) .
$$

Next we give a bound for II in case $\alpha \in\left(\frac{1}{2}, 1\right)$. We use the continuous embedding $H^{\alpha}\left(L_{1 / g}^{2}\right) \subset C\left(L_{1 / g}^{2}\right)$. 


$$
\begin{aligned}
& \mathrm{II}=2 \int_{s}^{T-t}\left|e^{\mu(\tau-s)} \int_{-\infty}^{0}[B(\tau, \sigma)-B(\tau-t, \sigma)] \dot{x}(\tau+\sigma) d \sigma\right|^{2} d \tau \\
& =2 \int_{s}^{T-t} e^{2 \mu(\tau-s)}\left(\int_{\tau}^{\tau+t} \int_{-\infty}^{0} \frac{\left|B_{t}(\rho, \sigma)\right|}{g(\sigma)^{1 / 2}}|\dot{x}(\tau+\sigma)| g(\sigma)^{1 / 2} d \sigma d \rho\right)^{2} d \tau \\
& \leq 2 \int_{s}^{T-t} e^{2 \mu(\tau-s)}\left\{\int_{\tau}^{\tau+t}\left(\int_{-\infty}^{0}\left|B_{t}(\rho, \sigma)\right|^{2} \frac{d \sigma}{g(\sigma)}\right)^{1 / 2}\right. \\
& \left.\left(\int_{-\infty}^{0}|\dot{x}(\tau+\sigma)|^{2} g(\sigma) d \sigma\right)^{1 / 2} d \rho\right\}^{2} d \tau \\
& \leq 2 t \int_{s}^{T-t} e^{2 \mu(\tau-s)} \int_{\tau}^{\tau-t}\left|B_{t}(\rho, \cdot)\right|_{L_{1 / g}^{2}}^{2} \int_{-\infty}^{0}|\dot{x}(\tau+\sigma)|^{2} g(\sigma) d \sigma d \rho d \tau \\
& \leq 2 t^{2}|B|_{H^{1+\alpha}\left(L_{1 / g}^{2}\right)}^{2} \int_{s}^{T-t} e^{2 \mu(\tau-s)}\left\{\int_{-\infty}^{s-\tau}|\dot{x}(\tau+\sigma)|^{2} g(\sigma) d \sigma\right. \\
& \left.+\int_{s-\tau}^{0}|\dot{x}(\tau+\sigma)|^{2} g(\sigma) d \sigma\right\} d \tau \\
& \leq 2 t^{2}|B|_{H^{1+\alpha}\left(L_{1 / g}^{2}\right)}^{2} \int_{s}^{T-t} e^{2 \mu(\tau-s)}\left\{\int_{-\infty}^{0}|\dot{\phi}(\sigma)|^{2} g(\sigma+s-\tau) d \sigma\right. \\
& \left.+\int_{s}^{\tau}|\dot{x}(\sigma)|^{2} g(\sigma-\tau) d \sigma\right\} d \tau \\
& \leq 2 t^{2}|B|_{H^{1+\alpha}\left(L_{1 / g}^{2}\right)}^{2} \int_{s}^{T-t} e^{2 \mu(\tau-s)}\left\{\left(\begin{array}{l}
\left.\sup _{[s-T, 0]} G\right) \\
{\left[s-\left.\phi\right|_{H_{g}^{1}} ^{2}\right.}
\end{array}\right.\right. \\
& \left.+(\underset{[s-T, 0]}{\operatorname{supess} g}) \int_{s}^{\tau}|\dot{x}(\sigma)|^{2} d \sigma\right\} d \tau \\
& \leq c t^{2}|B|_{H^{1+\alpha}\left(L_{1 / g}^{2}\right)}^{2}\left\{|\phi|_{H_{g}^{1}}^{2}+\frac{1}{|\mu|}|z|_{1, \mu}^{2}\right\} \text {. }
\end{aligned}
$$

Thus, for $\alpha \in\left(\frac{1}{2}, 1\right)$ we have

$$
\mathrm{II} \leq c t^{2}|B|_{H^{1+\alpha}\left(L_{1 / g}^{2}\right)}^{2}\left(|\phi|_{H_{g}^{1}}^{2}+\frac{1}{|\mu|}|z|_{1, \mu}^{2}\right) .
$$

Summarizing (3.39)-(3.41) we obtain

$$
\begin{aligned}
& {\left[\int_{-\infty}^{0} B(t, \sigma) \dot{x}(t+\sigma) d \sigma\right]_{\alpha, \mu}^{2}} \\
& \qquad \leq|B|_{H^{1+\alpha}\left(L_{1 / g}^{2}\right)}^{2}\left(\left(1+|\mu| e^{2|\mu|(T-s)}\right)|\phi|_{H_{g}^{1+\alpha}}^{2}+\frac{1}{|\mu|}|z|_{1+\alpha, \mu}^{2}\right) .
\end{aligned}
$$

(iii) Estimation of $A_{0}(t) x(t)$. We have

$$
\left[A_{0} x\right]_{\alpha, \mu}^{2}=\int_{0}^{1} t^{-1-2 \alpha} \beta(t) d t
$$


where

$$
\begin{aligned}
\beta(t):= & \int_{s}^{T-t}\left|e^{\mu(\tau-s)} A_{0}(\tau) x(\tau)-e^{\mu(\tau-s+t)} A_{0}(\tau+t) x(\tau+t)\right|^{2} d \tau \\
\leq & 2 \int_{s}^{T-t}\left|e^{\mu(\tau-s)} A_{0}(\tau) \phi(0)-e^{\mu(\tau-s+t)} A_{0}(\tau+t) \phi(0)\right|^{2} d \tau \\
& +2 \int_{s}^{T-t}\left|e^{\mu(\tau-s)} A_{0}(\tau) \int_{s}^{\tau} \dot{z}(\sigma) d \sigma-e^{\mu(\tau-s+t)} A_{0}(\tau+t) \int_{s}^{\tau+t} \dot{z}(\sigma) d \sigma\right|^{2} d \tau \\
= & : R+S .
\end{aligned}
$$

We estimate $R$ first using $1-e^{\mu t} \leq|\mu| t$ for $t \leq 0$,

$$
\begin{aligned}
R \leq & 4 \int_{s}^{T-t}\left|e^{\mu(\tau-s)} A_{0}(\tau) \phi(0)-e^{\mu(\tau-s)} A_{0}(\tau+t) \phi(0)\right|^{2} d \tau \\
& +4 \int_{s}^{T-t}\left|e^{\mu(\tau-s)}\left(1-e^{\mu t}\right) A_{0}(\tau+t) \phi(0)\right|^{2} d \tau \\
\leq & 4\left|A_{0}(\cdot)-A_{0}(\cdot+t)\right|_{L^{2}\left(s, T-t ; \mathbf{R}^{n \times n}\right)}^{2}|\phi(0)|^{2} \\
& +\left.4|\mu| t^{2}|\phi(0)|^{2} A_{0}\right|_{L^{2}} ^{2} .
\end{aligned}
$$

For $S$ we have the estimate

$$
\begin{aligned}
S \leq & 4 \int_{s}^{T-t}\left|e^{\mu(\tau-s)} A_{0}(\tau) \int_{s}^{\tau} \dot{z}(\sigma) d \sigma-e^{\mu(\tau-s+t)} A_{0}(\tau) \int_{s}^{\tau+t} \dot{z}(\sigma) d \sigma\right|^{2} d \tau \\
& +4 \int_{s}^{T-t}\left|\left(A_{0}(\tau)-A_{0}(\tau+t)\right) e^{\mu(\tau-s+t)} \int_{s}^{\tau+t} \dot{z}(\sigma) d \sigma\right|^{2} d \tau
\end{aligned}
$$$$
=: \mathrm{I}+\mathrm{II} \text {. }
$$

We first give a bound for II:

$$
\begin{aligned}
\mathrm{II} & \leq 4 \int_{s}^{T-t}\left[\left|A_{0}(\tau)-A_{0}(\tau+t)\right| \int_{s}^{\tau+t}|\dot{z}(\sigma)| e^{\mu(\sigma-s)} e^{\mu(\tau-\sigma+t)} d \sigma\right]^{2} d \tau \\
& \leq \frac{2}{|\mu|} \int_{s}^{T}\left|A_{0}(\tau)-A_{0}(\tau+t)\right|^{2} \int_{s}^{\tau+t}|\dot{z}(\sigma)|^{2} e^{2 \mu(\sigma-s)} d \sigma d \tau \\
& \leq \frac{2}{|\mu|} \int_{s}^{T} \int_{\max (s, \sigma-t)}^{T-t}\left|A_{0}(\tau)-A_{0}(\tau+t)\right|^{2}|\dot{z}(\sigma)|^{2} e^{2 \mu(\sigma-s)} d \tau d \sigma \\
& \leq \frac{2}{|\mu|}|z|_{1, \mu}^{2}\left|A_{0}(\cdot)-A_{0}(\cdot+t)\right|_{L^{2}\left(s, T-t ; \mathbf{R}^{n \times n}\right)}^{2} .
\end{aligned}
$$

Next we estimate I:

$$
\begin{aligned}
\mathrm{I} \leq & 8 \int_{s}^{T-t}\left|A_{0}(\tau)\left[e^{\mu(\tau-s)} \int_{s}^{\tau} \dot{z}(\sigma) d \sigma-e^{\mu(\tau-s+t)} \int_{s}^{\tau} \dot{z}(\sigma+t) d \sigma\right]\right|^{2} d \tau \\
& +8 \int_{s}^{T-t}\left|A_{0}(\tau) e^{\mu(\tau-s+t)} \int_{s}^{s+t} \dot{z}(\sigma) d \sigma\right|^{2} d \tau .
\end{aligned}
$$


In case $\alpha \in\left(0, \frac{1}{2}\right)$ we have

$$
\begin{aligned}
\mathrm{I} \leq & 8 \int_{s}^{T-t} \mid A_{0}(\tau)\left[\int_{s}^{\tau} e^{\mu(\sigma-s)} e^{\mu(\tau-\sigma)} \dot{z}(\sigma) d \sigma\right. \\
& \left.\quad-\int_{s}^{\tau} e^{\mu(\sigma-s+t)} e^{\mu(\tau-\sigma)} \dot{z}(\sigma+t) d \sigma\right]\left.\right|^{2} d \tau \\
& +8 \int_{s}^{T-t}\left(\left|A_{0}(\tau)\right| \int_{s}^{s+t} e^{\mu(\sigma-s)}|\dot{z}(\sigma)| e^{\mu(\tau-\sigma+t)} d \sigma\right)^{2} d \tau \\
\leq & \frac{4}{|\mu|}\left|A_{0}\right|_{L^{2}}^{2}\left|e^{\mu(\cdot-s)} \dot{z}(\cdot)-e^{\mu(\cdot-s+t)} \dot{z}(\cdot+t)\right|_{L^{2}\left(s, T-t ; \mathbf{R}^{n}\right)}^{2} \\
& +\frac{4}{|\mu|}\left|A_{0}\right|_{L^{2}}^{2} \int_{s}^{s+t} e^{2 \mu(\sigma-s)}|\dot{z}(\sigma)|^{2} d \sigma .
\end{aligned}
$$

Now we take $\alpha \in\left(\frac{1}{2}, 1\right)$ :

$$
\begin{aligned}
\mathrm{I} \leq & \frac{4}{|\mu|}\left|A_{0}\right|_{L^{2}}^{2}\left|e^{\mu(\cdot-s)} \dot{z}(\cdot)-e^{\mu(\cdot-s+t)} \dot{z}(\cdot+t)\right|_{L^{2}\left(s, T-t ; \mathbf{R}^{n}\right)}^{2} \\
& +8\left|A_{0}\right|_{L^{\infty}}^{2}\left|e^{\mu(\cdot-s)} \dot{z}\right|_{L^{\infty}}^{2} \int_{s}^{T-t}\left(\int_{s}^{s+t} \mid e^{\mu(\tau-\sigma+t)} d \sigma\right)^{2} d \tau \\
\leq & \frac{4}{|\mu|}\left|A_{0}\right|_{L^{2}}^{2}\left|e^{\mu(\cdot-s)} \dot{z}(\cdot)-e^{\mu(\cdot-s+t)} \dot{z}(\cdot+t)\right|_{L^{2}\left(s, T-t ; \mathbf{R}^{n}\right)}^{2} \\
& +\frac{4 c}{|\mu|}\left|A_{0}\right|_{H^{\alpha}}^{2}|\dot{z}|_{H_{\mu}^{\alpha}}^{2} t^{2} .
\end{aligned}
$$

Here we have used the continuous embeddings $H^{\alpha} \subset C, \alpha>\frac{1}{2}$. Summarizing (3.42)-(3.46) and using Lemma $1.12(\mathrm{a})$ (in case $\alpha \in\left(\frac{1}{2}, 1\right)$ ) we obtain

$$
\left[A_{0} x\right]_{\alpha, \mu} \leq c\left|A_{0}\right|_{H^{\alpha}}\left((1+|\mu|)^{1 / 2}|\phi|_{H_{g}^{1}}+\frac{1}{|\mu|^{1 / 2}}|z|_{1+\alpha, \mu}\right) .
$$

As mentioned earlier, the estimates for the remaining terms are - although somewhat tedious - essentially analogous to those we discussed here.

For $m \geq 2$ we use (3.34) and inequalities of the same type as in case $m=1$.

\section{REFERENCES}

1. A. V. Balakrishnan, Applied functional analysis, Springer, New York, 1976.

2. H. T. Banks, J. A. Burns and E. M. Cliff, Parameter estimation and identification for systems with delays, SIAM J. Control Optim. 19 (1981), 791-828.

3. H. T. Banks and P. L. Daniel, Estimation of delays and other parameters in nonlinear functional differential equations, SIAM J. Control Optim. 21 (1983), 895-915.

4. J. A. Burns., T. L. Herdman and H. W. Stech, Linear functional differential equations as semigroups on product spaces, SIAM J. Math. Anal. 14 (1983), 98-116.

5. P. L. Butzer and H. Berens, Semi-groups of operators and approximation, Springer, Berlin, 1967.

6. J. K. Hale and J. Kato, Phase space for retarded equations with infinite delay, Funkcial. Ekvac. 21 (1978), 11-41.

7. E. Hewitt and K. Stromberg, Real and abstract analysis, Springer, Heidelberg, 1965.

8. F. Kappel, Approximation of neutral functional differential equations in the state space $\mathbf{R}^{n} \times L^{2}$, Colloq. Math. Soc. Janos Bolyai, 30, Qualitative Theory of Differential Equations, Vol. I (M. Farkas, ed.), Janos Bolyai Math. Soc. and North-Holland, Amsterdam, 1982, pp. 463-506. 
9 F. Kappel and K. Kunisch, An approximation scheme for parameter estimation in infinite delay equations of Volterra type. Numerical results, Technical Report, Institutes for Mathematics, University and Technical University of Graz, No. 51, 1984.

10. F. Kappel and W. Schappacher, Some considerations to the fundamental theory of infinite delay equations, J. Differential Equations 37 (1980), 141-183.

11. J. L. Lions and E. Magenes, Nonhomogeneous boundary value problems and applications, Vol. I, Springer, Heidelberg, 1972.

12. I. Lasiecka and A. Manitius, Differentiability and convergence rates of approximating semigroups for retarded functional differential equations, SIAM J. Numer. Anal. (to appear).

13. C. Sadosky, Interpolation of operators and singular integrals. An introduction to harmonic analysis, Dekker, New York, 1979.

14. K. Schumacher, Remarks on semilinear partial functional equations with infinite delay, J. Math. Anal. Appl. 80 (1981), 261-290.

15. O. Staffans, Extended initial and forcing function semigroups generated by a functional equation, SIAM J. Math. Anal. 16 (1985), 1034-1048.

16. __ Semigroups generated by a neutral functional differential equation, SIAM J. Math. Anal. 17 (1986), 46-57.

17. $\mathrm{H}$. Triebel, Interpolation theory, function spaces, differential operators, North-Holland, Amsterdam, 1978.

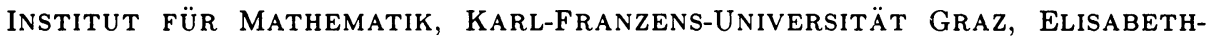
Strasse 16, A-8010 Graz, Austria

Institut für Mathematik, Technische Universität Graz, Kopernikusgasse 24, A-8010 Graz, Austria 